\title{
Valuation Techniques for Complex Space Systems: An Analysis of a Potential Satellite Servicing Market
}

\author{
by \\ Michelle E. McVey \\ B.A. in Management Engineering (2000) \\ Claremont McKenna College \\ B.S. in Aerospace Engineering (2000) \\ University of California, Los Angeles
}

Submitted to the Department of Aeronautics and Astronautics Engineering in Partial Fulfillment for the Degree of

Master of Science in Aeronautics and Astronautics

at the

Massachusetts Institute of Technology

June 2002

(C) 2002 Massachusetts Institute of Technology

All Rights Reserved

Signature of Author

Department of Aeronautics and Astronautics

Certified by

Dr. Joyce Warmkessel

Senior Lecturer, Department of Aeronautics and Astronautics

Thesis Advisor

Accepted by.

Wallace E. Vander Velde

Professor of Aeronautics and Astronautics

Chair, Committee on Graduate Students 



\title{
Valuation Techniques for Complex Space Systems: An Analysis of a Potential Satellite Servicing Market
}

\author{
by
}

\author{
Michelle E. McVey
}

\author{
Submitted to the Department of Aeronautics and Astronautics Engineering \\ on May 10, 2002 in Partial Fulfillment of the \\ Requirements for the Degree of Master of Science in
} Aeronautics and Astronautics

\begin{abstract}
Current financial valuation techniques fail to capture several important aspects of technical projects, including flexibility and the interface between economics and technology. Additionally, valuations rarely aid in the process of determining which service or product provides value to both the client and the provider.

This thesis presents a new valuation framework that accounts for these downfalls by breaking the valuation analysis into two distinct parts: the client's value and the provider's value. The client value analysis is a necessary step in determining the provider's value, as it provides the basis for the revenue the provider will generate as well as an idea of which type of service or product provides the most value to the client. As a viable market does not exist without both a client and a provider, it is necessary to look at a project from both perspectives.

The valuation framework is used to analyze the commercial geosynchronous (GEO) satellite servicing market. Several approaches of servicing are examined and compared to current satellites and electric propulsion, which are considered the most probable competition for servicing. The analysis indicates that two primary types of satellite servicing provide value to both the client and provider - using direct GEO insertion and either a tug spacecraft to perform the client's North-South stationkeeping maneuvers or a refueling vehicle enable the spacecraft to perform its own stationkeeping maneuvers.
\end{abstract}

Thesis Supervisor: Joyce Warmkessel

Title: Senior Lecturer in Aeronautics and Astronautics 



\section{ACKNOWLEDGEMENTS}

This thesis would be nowhere near complete without acknowledging the many people who made it possible. I would like to express my deepest gratitude to the following:

To my Mom and Dad. Nothing I can say can do justice to what you have given me throughout the past 25 years. Your support and encouragement for all of my endeavors has allowed me to do things I never thought possible. As role models, you have set the bar very high and for that I am eternally grateful. Thank you.

To my advisor, Joyce Warmkessel. Thank you for your guidance and for allowing me the freedom to explore the area of research I found most fulfilling. I sincerely appreciate your advice on research, career choices, and life in general. These things will stick with me long after the details of this thesis have slipped away.

To the Lean Aerospace Initiative (LAI) Faculty, Students, and Staff. I have sincerely enjoyed being a part of LAI and am incredibly grateful for the support and opportunities that I have received. Thanks for making each day interesting. I feel extremely lucky to be surrounded by such motivated, intelligent, and entertaining people.

To Andy Turner. Thank you for working with me last summer and for the support you have provided for my research since then. Your enthusiasm for your work, especially Aquarius, was contagious and got me through many days and nights with my computer.

To Gert VanOmmering. Thank you for giving me the opportunity to work at Space Systems/LORAL. I had a wonderful summer and managed to learn a lot in the process. I also appreciate you tracking me down on your occasional trips to Boston to offer support for both research and career advice.

To Jacob Markish. Thank you for your unconditional friendship, your amazing ability to listen, all of the time you spent helping me throughout the last four semesters, making the long nights at lab bearable, and for being an awesome editor. Your presence made my time at MIT immeasurably more enjoyable.

To my favorite climbing buddy, Rob Scott. Your friendship has been invaluable. Thank you for dragging me up to the mountains and helping me maintain my sanity in the process. Here's to a great trip to Bolivia and some great climbs out West!

To Noelle Bachas. Thank you for being you. You brighten the lives of everyone you touch and I feel very fortunate to be one of those lucky people.

To the rest of my friends and family. Thank you for always being there and for being understanding about the unsent emails, unmade phone calls, and unspent time.

To the employees of Space Systems/LORAL. Thank you for making my summer at LORAL enjoyable, memorable, and a great learning experience. The support and environment you provided for my research is greatly appreciated. 



\section{TABLE OF CONTENTS}

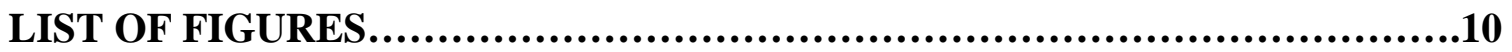

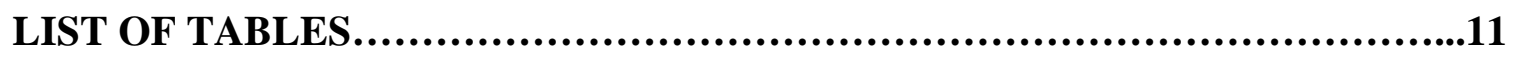

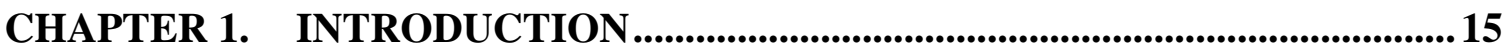

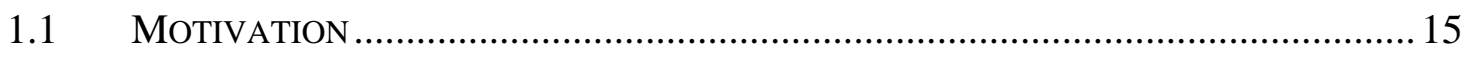

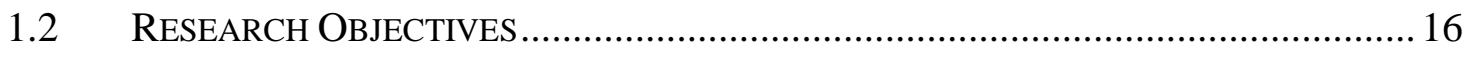

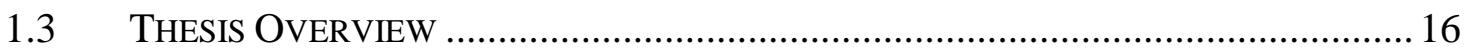

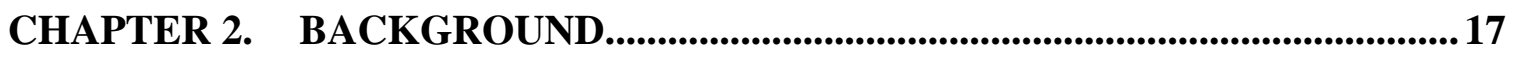

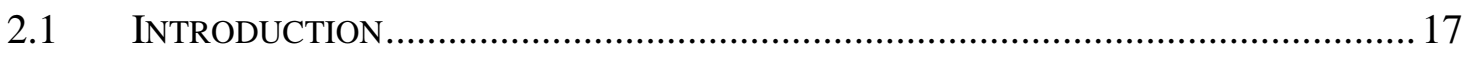

2.2 Aquarius: A New Perspective on Launching Consumables ..................... 17

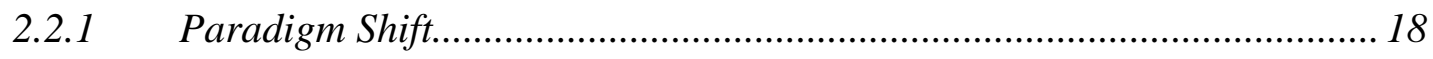

2.2.2 Aquarius Conceptual Design and Potential Markets............................... 18

2.3 FinANCIAL VALUATION TOOLS....................................................................22

2.3.1 What is the Real Options Approach? ………………………....................2 22

2.3.2 How Does Real Options Compare to Standard Valuation Techniques? .. 23

2.3.3 Where can the Real Options Approach be Utilized?.................................. 24

2.3.4 Where can Real Options be Utilized in the Aerospace Industry? .............. 25

2.3.5 Valuations: Using the Binomial Real Options Approach ........................ 26

2.3.6 Extension to the Black-Scholes Formula ……………………………........ 34

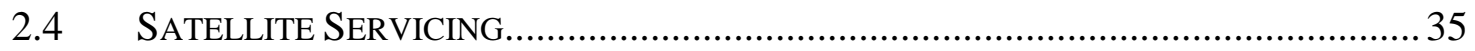

2.4.1 History of Servicing......................................................................... 35

2.4.2 Current servicing program: Orbital Express........................................... 37

\section{CHAPTER 3. VALUATION: INTERFACE BETWEEN TECHNOLOGY} AND ECONOMICS ..................................................................................................................39

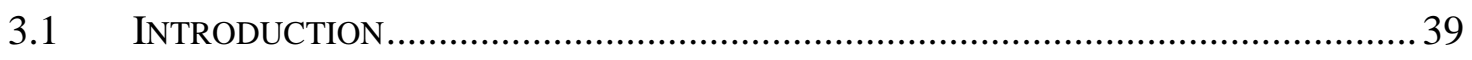

3.2 WHY ARE NEW VALUATION TECHNIQUES NECESSARY? ………............................ 39

3.2.1 Doing the Right Job............................................................................... 39

3.2.2 Economics and Technology in Valuations .............................................. 40 


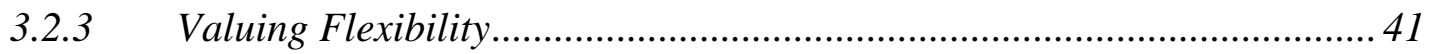

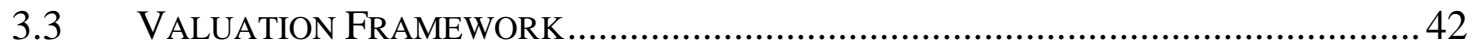

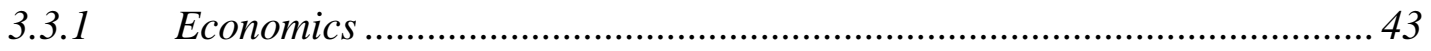

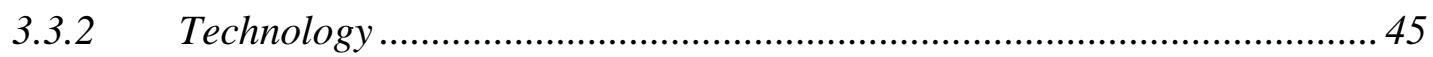

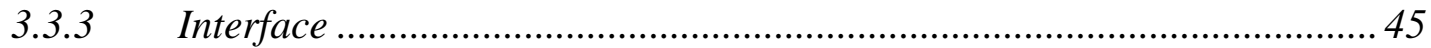

3.4 APPLYING THE VALUATION FRAMEWORK ............................................................ 46

3.4.1 Is there a potential market? …………………........................................ 47

3.4.2 What does the market want? ..................................................................... 48

3.4.3 What is the value of the service to the client? ............................................ 48

3.4.4 How much will it cost to provide service? ................................................ 49

3.4.5 What is the value of the project to the provider? ........................................ 49

3.4.6 Learning by applying the valuation framework ......................................... 50

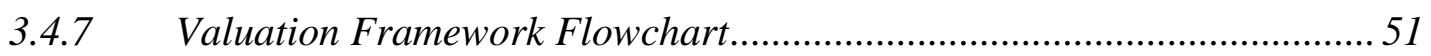

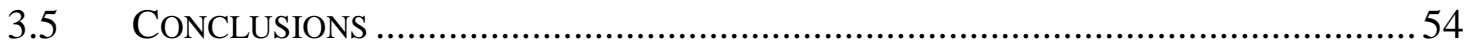

\section{CHAPTER 4. SATELLITE SERVICING: CLIENT VALUE ANALYSIS...........55}

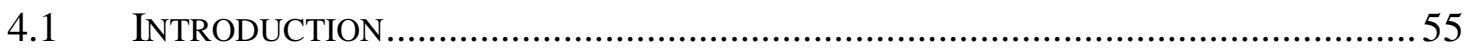

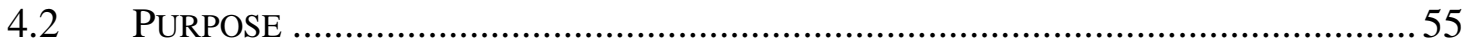

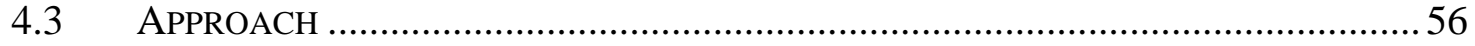

4.3.1 Important Technological Aspects of Each Case ....................................... 60

4.3.2 Transforming the Baseline Satellite to the Case Configurations............... 64

4.3.3 Baseline Calculations for Subtraction or Addition of Parts ...................... 67

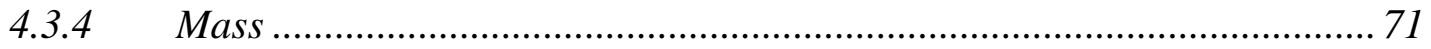

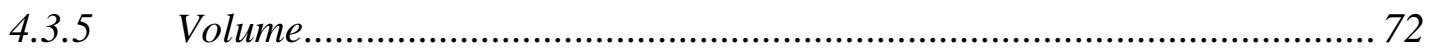

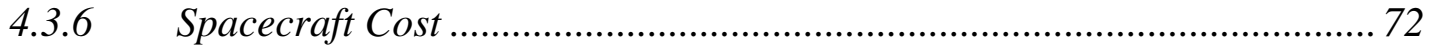

4.3.7 Launch Vehicle Cost....................................................................... 73

4.3.8 Limiting Factor Analysis...................................................................... 74

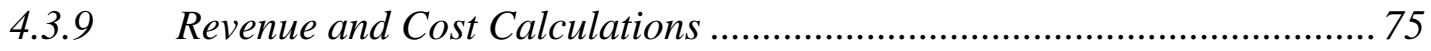

4.3.10 Discounted Cash Flow Analysis.......................................................... 76

4.3.11 Option to Extend Life of Satellite ........................................................... 77

4.3.12 Flexibility to relocate satellite and capture other markets ....................... 79

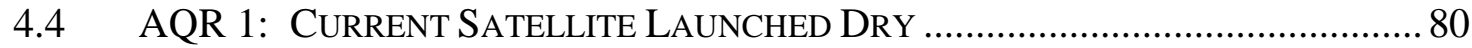




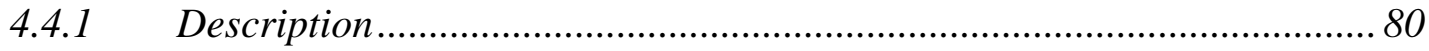

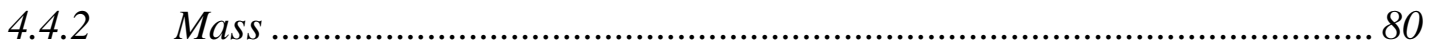

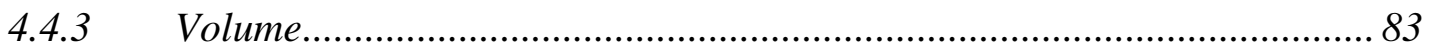

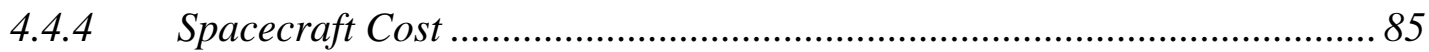

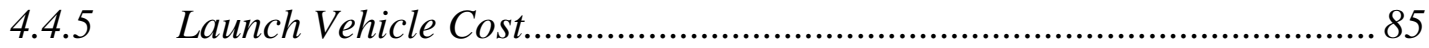

4.4.6 Limiting Factor Analysis................................................................... 85

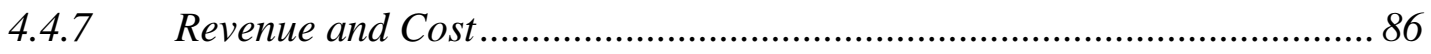

4.4.8 Discounted Cash Flow and Options .....................................................8 86

4.5 AQR 2: LaUnCh to STAGING ORbit AND TUg For ORbit Raising (OR) AND

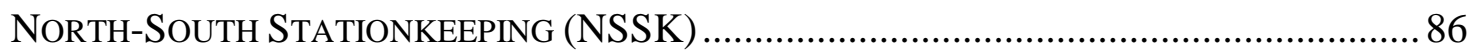

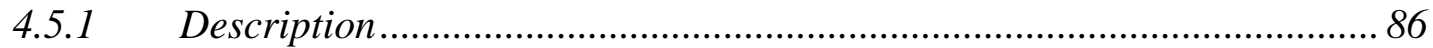

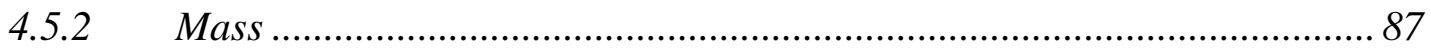

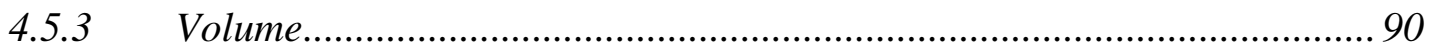

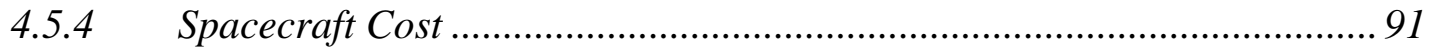

4.5.5 Launch Vehicle Cost.......................................................................... 91

4.5.6 Limiting Factor Analysis................................................................ 92

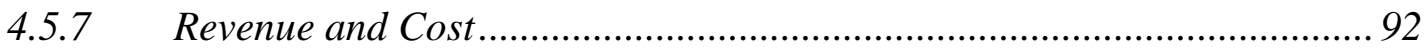

4.5.8 Discounted Cash Flow and Options .................................................... 93

4.6 AQR 3: DIRECT GEO INSERTION (DGI) WITH TUg FOR NORTH-SOUTH

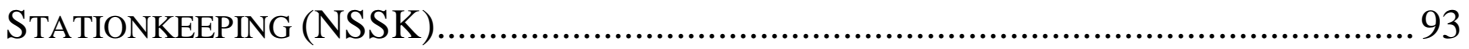

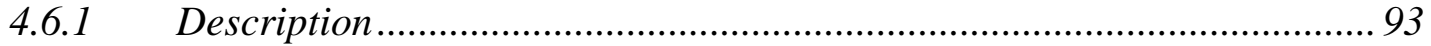

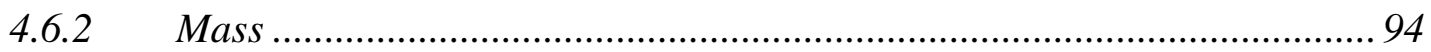

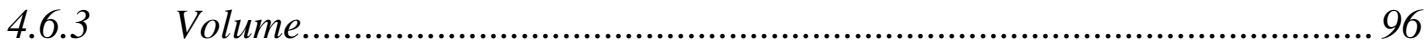

4.6.4 Spacecraft Cost ................................................................................ 97

4.6.5 Launch Vehicle Cost........................................................................97

4.6.6 Limiting Factor Analysis................................................................... 98

4.6.7 Revenue and Cost ......................................................................... 98

4.6.8 Discounted Cash Flow and Options ....................................................98

4.7 AQR 4: DiRECT GEO INSERTION WITH JUST IN TIME REFUELING..................... 99

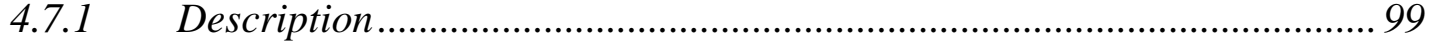

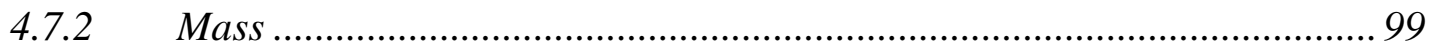




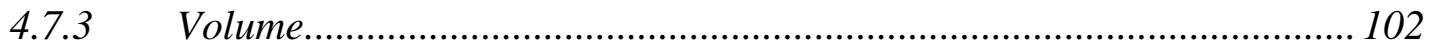

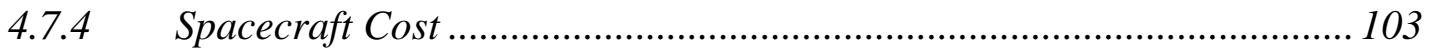

4.7.5 Launch Vehicle Cost........................................................................... 103

4.7.6 Limiting Factor Analysis.............................................................. 104

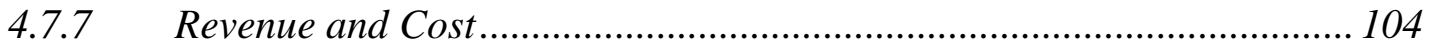

4.7.8 Discounted Cash Flow and Options ................................................... 104

4.8 AQR 5: DIRECT GEO INSERTION WITH LOWER FREQUENCY REFUELING ...... 105

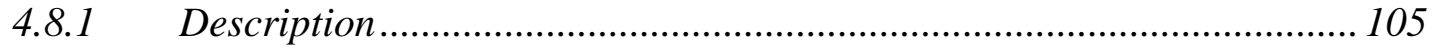

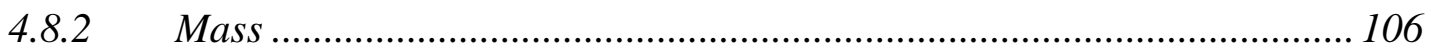

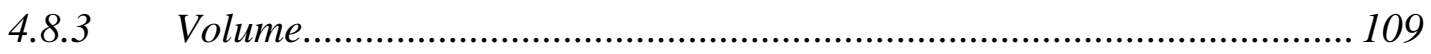

4.8.4 Spacecraft Cost ........................................................................... 110

4.8.5 Launch Vehicle Cost............................................................................ 110

4.8.6 Limiting Factor Analysis............................................................ 110

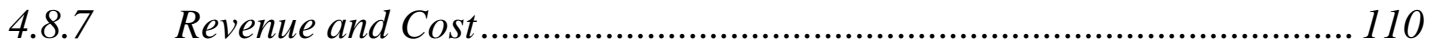

4.8.8 Discounted Cash Flow and Options ................................................. 111

4.9 AQR 6: LAUnCH TO Staging, USE Tug FOR ORBIT RAISING, AND ELECTRIC PROPULSION (EP) FOR STATIONKEEPING .......................................................... 111

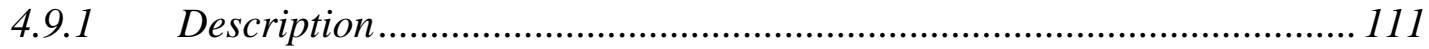

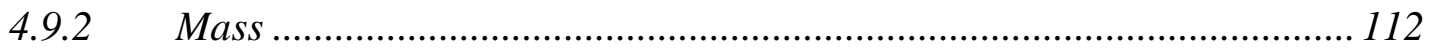

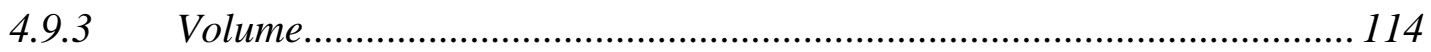

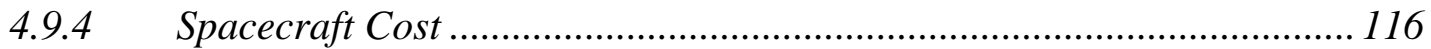

4.9.5 Launch Vehicle Cost.......................................................................... 116

4.9.6 Limiting Factor Analysis.............................................................. 116

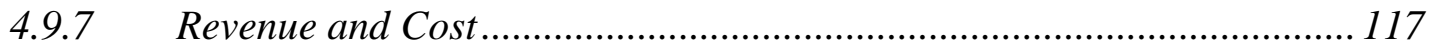

4.9.8 Discounted Cash Flow and Options ................................................ 117

4.10 COMP 1: Direct GEO InSERTION With EleCtric PROPUlSION FOR

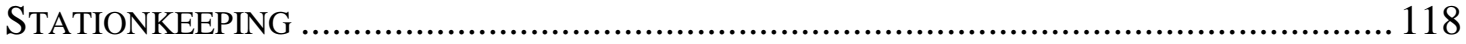

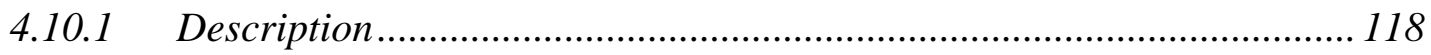

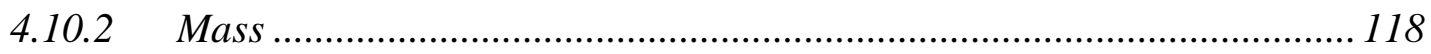

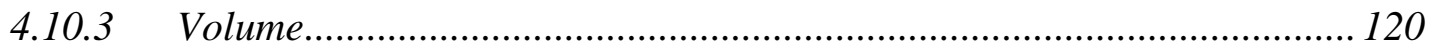

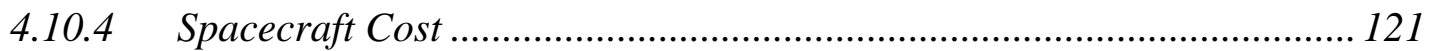


4.10.5 Launch Vehicle Cost.......................................................................... 122

4.10.6 Limiting Factor Analysis................................................................. 122

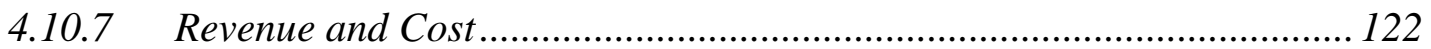

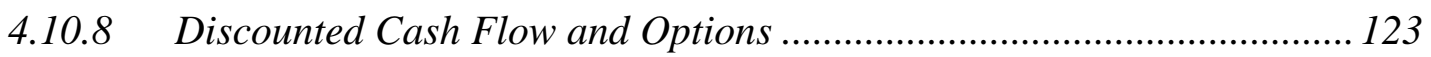

4.11 Comp 2: Launch to STAging Orbit AND USE Electric Propulsion For

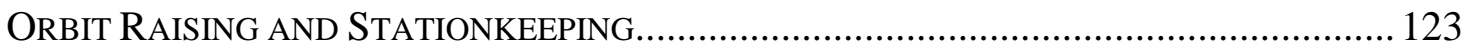

4.11.1 Description ................................................................................... 123

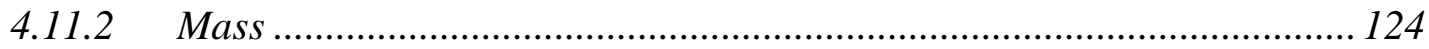

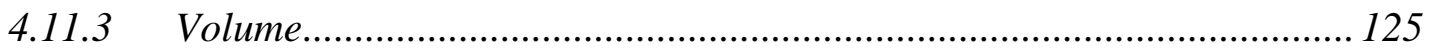

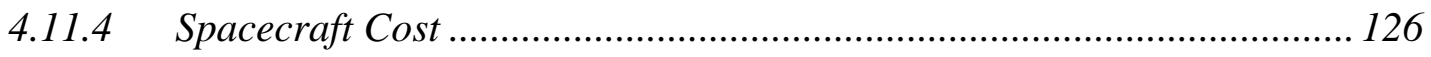

4.11.5 Launch Vehicle Cost...................................................................... 127

4.11.6 Limiting Factor Analysis................................................................ 127

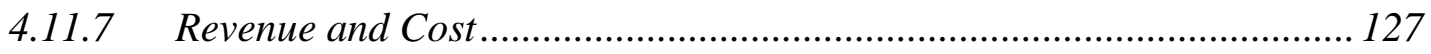

4.11.8 Discounted Cash Flow and Options ............................................... 128

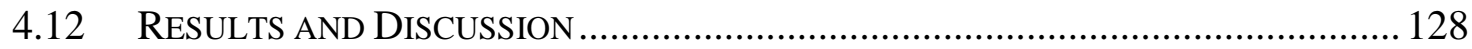

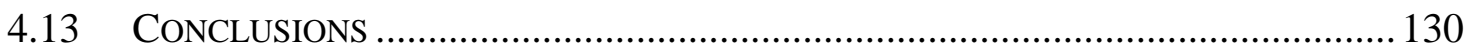

\section{CHAPTER 5. SATELLITE SERVICING: PROVIDER VALUE ANALYSIS.. 133}

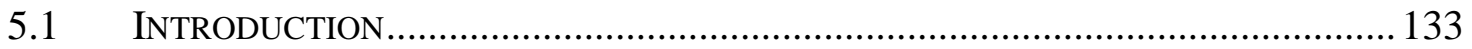

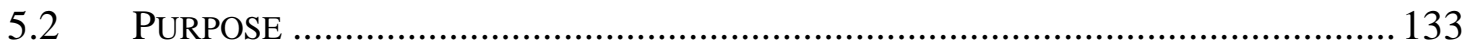

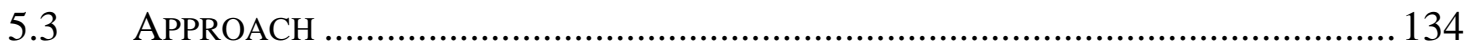

5.3.1 Estimating Potential Revenues from Servicing ...................................... 134

5.3.2 Estimating Cost of Servicing ................................................................ 135

5.3.3 Discounted Cash Flow to Determine Provider's NPV .......................... 136

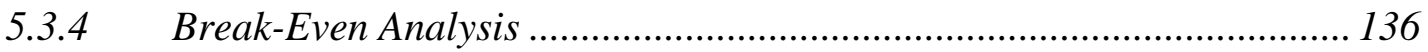

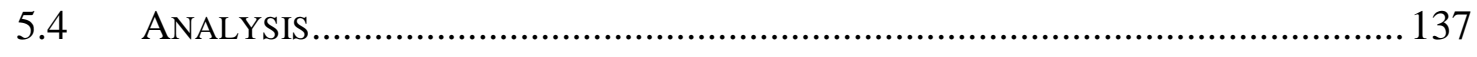

5.4.1 Estimating Potential Revenues from Servicing ..................................... 137

5.4.2 Estimating Costs of Servicing …………………................................. 140

5.4.3 Discounted Cash Flow to Determine Provider's NPV ........................... 146

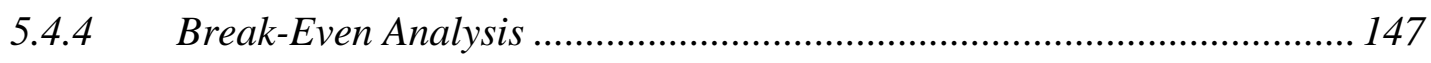

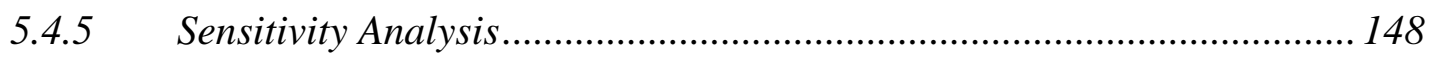

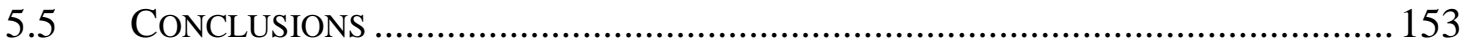


CHAPTER 6. CONCLUSION_..................................................................................... 155

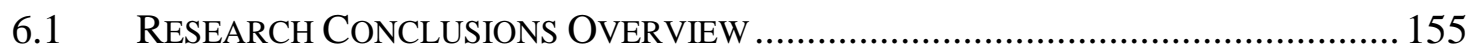

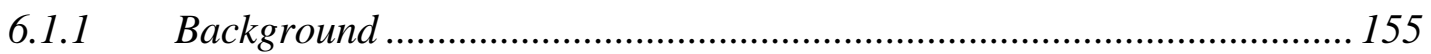

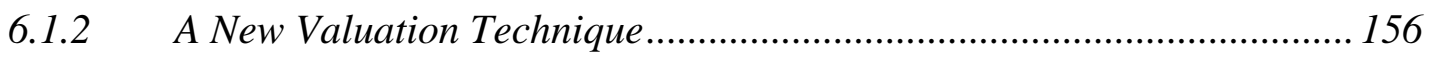

6.1.3 Client Value Analysis for Satellite Servicing ......................................... 156

6.1.4 Provider Value Analysis for Satellite Servicing..................................... 156

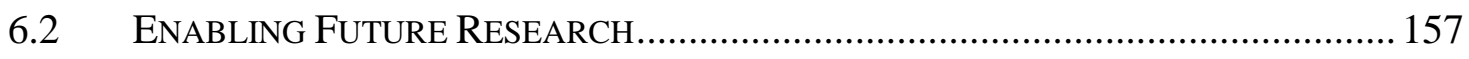

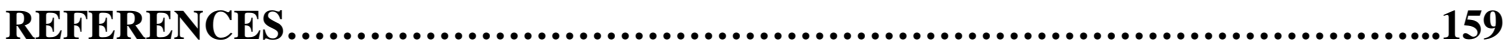

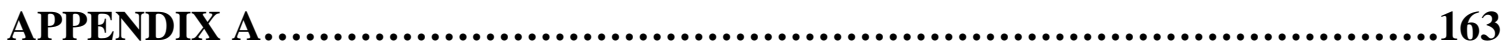




\section{LIST OF FIGURES}

Figure 2-1 Aquarius Low Reliability Launch Vehicle Concept .................................... 19

Figure 2-2 Aquarius Launch Vehicle Layout........................................................ 20

Figure 2-3 Aquarius Mission Profile ................................................................... 21

Figure 2-4 Predicted Market Outcomes for Year 1 .................................................... 27

Figure 2-5: Decision Tree used for Decision Analysis and Real Options Valuation........ 29

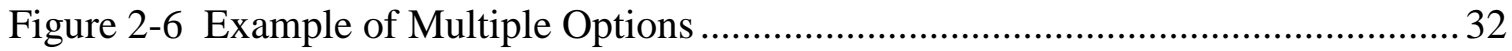

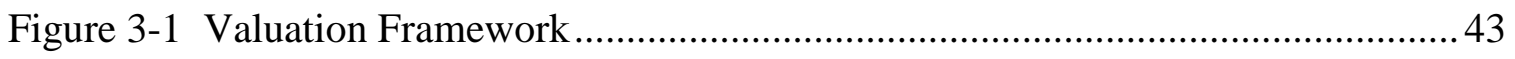

Figure 3-2 Example of Scenario Where Servicing Market is Not Feasible....................51

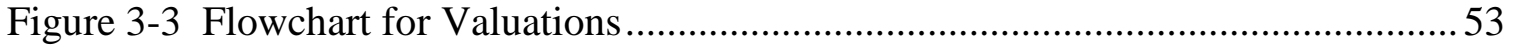

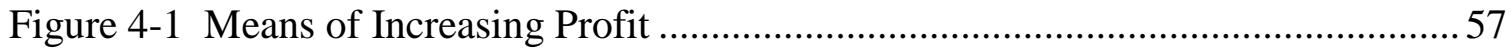

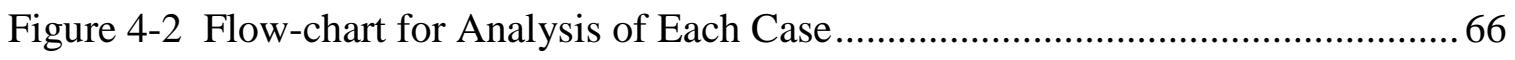

Figure 5-1 Servicing Architecture A ................................................................ 143

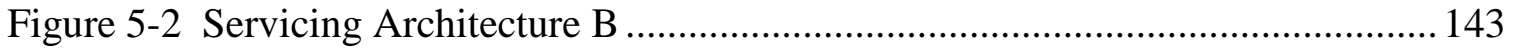

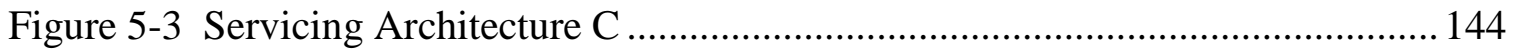

Figure 5-4 Sensitivity of Provider's NPV to \% of Client's Value Paid to Provider...... 148

Figure 5-5 Sensitivity of Provider NPV to Servicing Architecture Cost ...................... 150

Figure 5-6 Sensitivity of Servicing Provider's NPV to Fuel Delivery Costs ................ 151

Figure 5-7 Sensitivity of Provider's NPV to Discount Rate ...................................... 152 


\section{LIST OF TABLES}

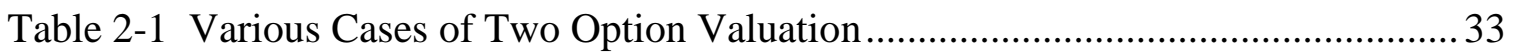

Table 4-1 GEO Servicing and Baseline Configuration Cases.....................................59

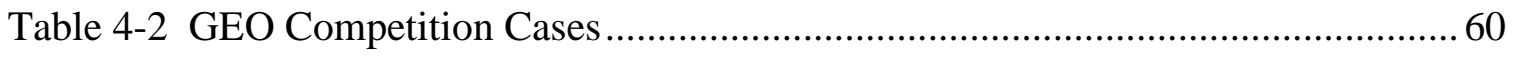

Table 4-3 Value of Satellite Servicing from Client Perspective ................................. 129

Table 5-1 Price Client is Willing to Pay for Servicing ............................................ 139

Table 5-2 Architecture Groups and Associated Cost Estimates .................................. 140

Table 5-3 Necessary Launches for Each Servicing Architecture Group ...................... 141

Table 5-4 Fuel Delivery Costs For Client Maneuvers Per Client Spacecraft ............... 145

Table 5-5 Fuel Delivery Costs for Support Maneuvers Per Client Spacecraft ............... 146

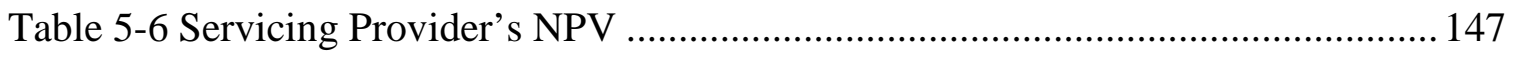

Table 5-7 Break-Even Analysis for Servicing Provider.......................................... 147 


\section{Chapter 1. Introduction}

\subsection{Motivation}

The decision of whether or not to pursue a new large-scale project is crucial to the success of any company in the aerospace industry. To make this decision, many aspects of the project must be considered, including both its technical and economic feasibility. These two aspects of feasibility are generally intertwined. For example, a project may be technologically feasible if a company is willing to spend exorbitant amounts of money on development. In this respect, the project may make technological sense, but its financial viability is compromised by the development costs.

Projects are often undertaken without sufficient understanding of what the most valuable product is. This happens for several reasons. First, traditional valuation techniques do not capture the interactions between technology and economics. Also, they tend to underestimate project value by neglecting the inherent flexibility found in most projects. Finally, traditional valuation techniques do not shed light on what the "right" product is.

One type of project that suffers from the shortcomings traditional valuations is the geosynchronous (GEO) satellite servicing. There exists a fairly large body of research in the area of satellite serving. Some studies have examined the technical aspects of servicing. Others have attempted to capture the value of servicing, by applying both traditional and more recently developed valuation techniques. However, the viability of 
the commercial GEO satellite servicing market has yet to be examined from a valuation perspective that accounts for both the technology and economics associated with servicing and determines which servicing configurations and architectures provide the most value to both the potential client and provider.

\subsection{Research Objectives}

In response the motivation above, this research has two primary objectives:

- Develop a valuation framework that accounts for the technical aspects, the economic aspects, and the flexibility of the project; and sheds light on what the "right" product is.

- Apply this framework to the GEO satellite servicing market to determine its viability from the perspective of both the potential client and provider.

\subsection{Thesis Overview}

Chapter 2 presents the background for this research. First, it introduces a new launch vehicle concept, Aquarius, which was the initial motivation for the analysis of the commercial GEO satellite market. Next, it presents an overview of real options, a technique used to value the flexibility in projects. Finally, it presents a brief overview of previous and ongoing research on satellite servicing. Chapter 3 presents a new valuation framework, which incorporates the technical and economic aspects of a potential project, as well as the flexibility of the project, and a determination of what the "right" product is. Chapter 4 and 5 present the application of this framework to the commercial GEO satellite servicing market. Chapter 4 focuses on the value servicing provides to the potential client, while Chapter 5 uses the information from Chapter 4 to determine the value of the market from the perspective of the servicing provider. Chapter 6 presents the conclusions of the research as well as future research opportunities. 


\section{Chapter 2. Background}

\subsection{Introduction}

This chapter serves to introduce background on three key aspects of the research. First, it introduces a new launch vehicle concept, Aquarius, the initial motivation for the analysis of the commercial GEO satellite market. Next, it presents an overview of real options, a technique used to value the flexibility in projects. Finally, it presents a brief overview of previous and current research on satellite servicing.

\subsection{Aquarius: A New Perspective on Launching Consumables}

This section introduces a new launch vehicle concept, Aquarius. The primary idea behind the Aquarius is to reduce the cost of launching low-cost items, such as fuel or water, by designing a launch vehicle with less emphasis on reliability. First, the rationale behind the paradigm shift Aquarius represents is discussed. Next, the basic design of the launch vehicle is presented. Finally, a brief description of possible markets for Aquarius is presented. As several articles about Aquarius are published, this section will not present an in-depth discussion of Aquarius. Rather, it will highlight the primary concepts behind Aquarius. This thesis is not intended to endorse the Aquarius launch vehicle, rather, Aquarius is presented as an interesting approach to reducing launch costs and used it as a way to examine the satellite servicing market. 


\subsubsection{Paradigm Shift}

In recent years much of the focus on improving launch capabilities has been on increasing reliability. This is because launches are very expensive and the cargo aboard launch vehicles is very valuable, making a launch failure very costly. Given this environment, why would someone propose to develop a low reliability launch vehicle?

Launch vehicles today cost almost as much as the spacecraft they carry. Generally about half of the mass of a launched spacecraft is propellant mass, which is clearly much less valuable than the spacecraft itself. One concept for reducing launch costs is to launch high value items on high reliability launch vehicles, and relegate low value items to lowcost, low-reliability launch vehicles. This would reduce the cost of launch by allowing spacecraft to be launched without fuel and put on smaller launch vehicles or to be launched with an increased number of spacecraft on one launch vehicle. Another option would be to increase the payload onboard a given spacecraft, increasing its capabilities for the same launch cost.

\subsubsection{Aquarius Conceptual Design and Potential Markets}

As a low-reliability, moderate-performance launch vehicle Aquarius, expects up to onethird of its launches to fail. As a result, it is not feasible to launch the vehicle from a launch pad because the pad would likely have to be replaced often. Instead, Aquarius is launched from the water. (See Figure 2-1) 


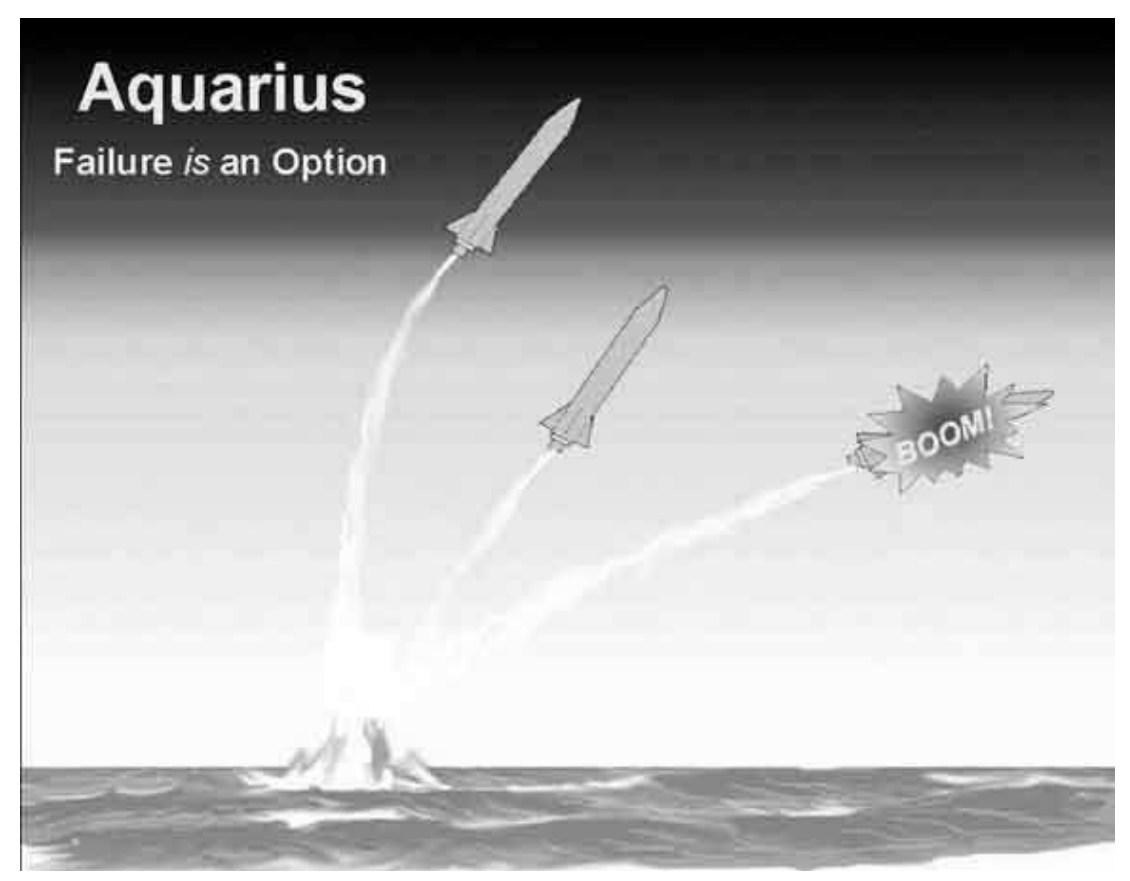

\section{Figure 2-1 Aquarius Low Reliability Launch Vehicle Concept ${ }^{1}$}

Although floating launches are certainly not common today, they have been demonstrated several times in the past (Draim, 1997).

The Aquarius launch vehicle uses liquid hydrogen and oxygen as its propellants. Its total liftoff mass is approximately 130 tons, with a payload of approximately 1 ton. $^{2}$ Its configuration is show in Figure 2-2.

\footnotetext{
${ }^{1}$ Adapted from Aquarius presentation, by Andrew Turner, Space Systems/LORAL.

${ }^{2}$ For a more in-depth technical description of Aquarius see (Turner, Ref. 3, 2000).
} 


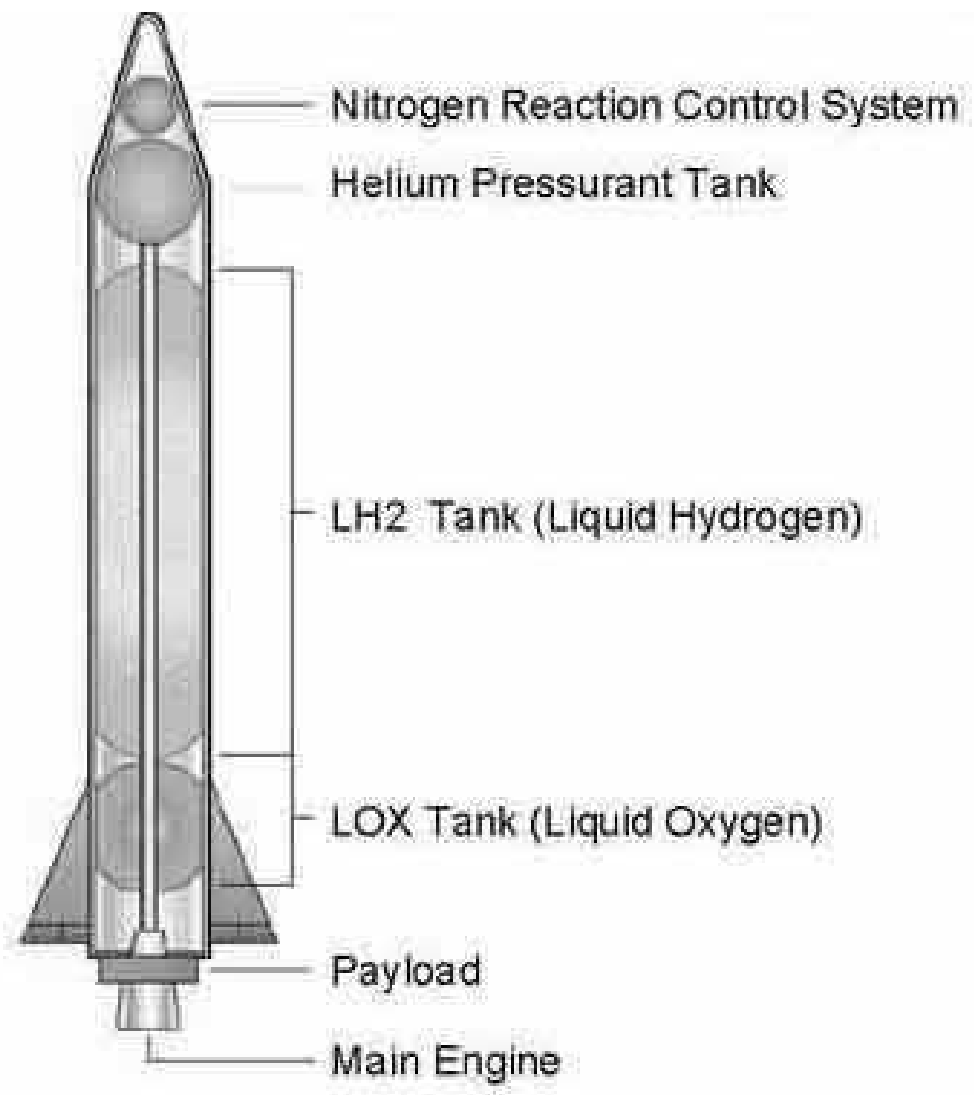

\section{Figure 2-2 Aquarius Launch Vehicle Layout ${ }^{3}$}

The Aquarius vehicle is designed to take low intrinsic value consumables to high value clients. Thus, it needs a high reliability interface between itself and client spacecraft. This is provided by a space "tugboat" which docks with Aquarius, removes the payload, and then returns to an on-orbit storage depot where the payload is stored until the client is ready to receive it. This process is illustrated in Figure 2-3.

\footnotetext{
${ }^{3}$ Adapted from (Turner 1, 2001)
} 


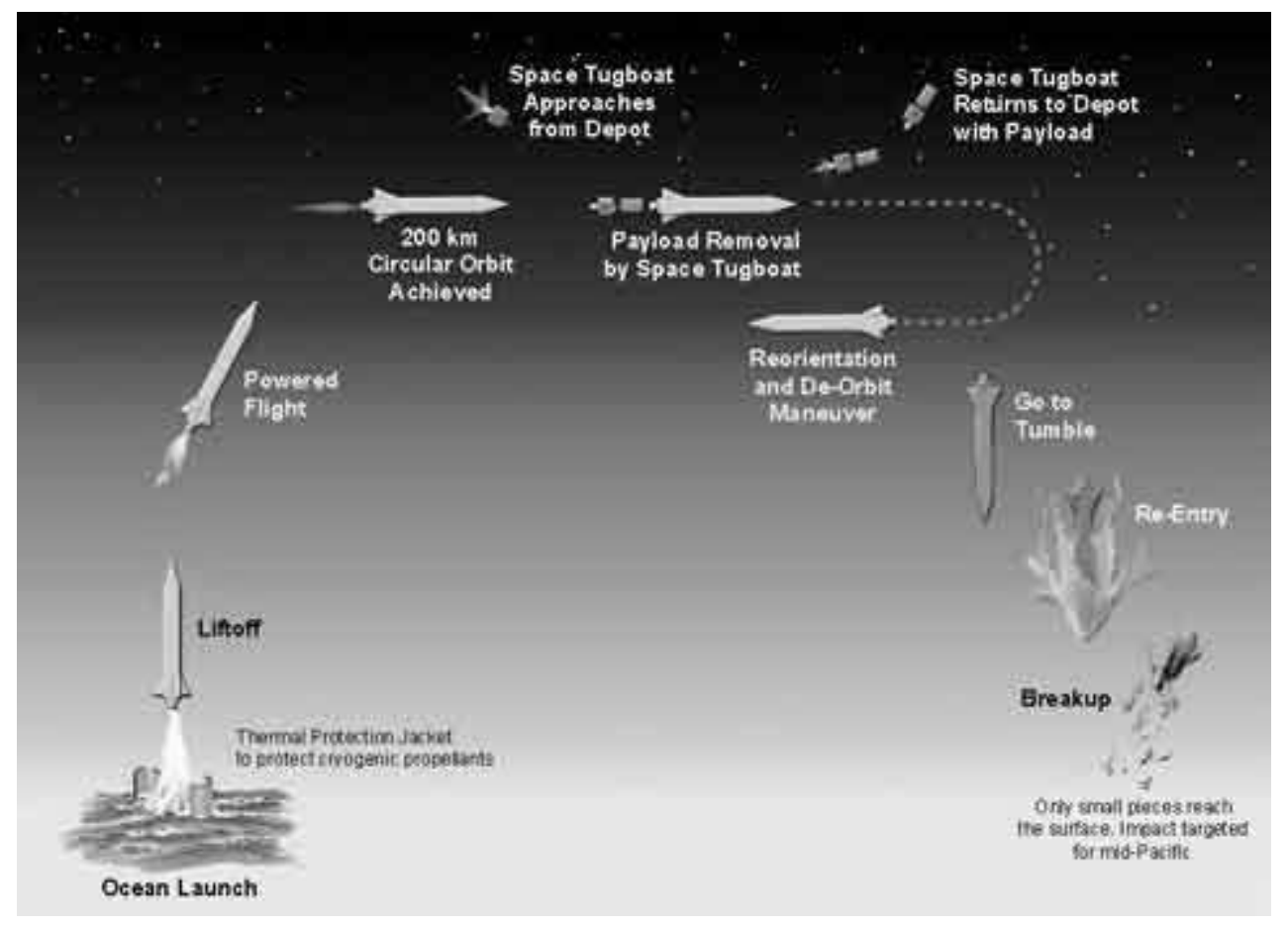

\section{Figure 2-3 Aquarius Mission Profile ${ }^{4}$}

The Aquarius vehicle is designed primarily to minimize cost, even if that leads to degrading its performance and reliability. By reducing the reliability, it would be possible to reduce extensive testing or rework on the vehicle or in-depth investigations of failures, which are very costly. Additionally, by removing performance as a primary driver, costly turbo-pumps become unnecessary. Instead, they can be replaced by a low cost pressure-fed propulsion system (Turner, Ref. 1, 2001).

As much of the cargo that is launched to space is inherently low value, there are several possible markets for Aquarius. These markets include, but are not limited to:

- International Space Station (re-supply: water, food, duct tape, etc.)

- Satellite servicing market (fuel delivery)

- Military operations (fuel delivery)

\footnotetext{
${ }^{4}$ Adapted from "Aquarius Launch Vehicle: Failure is an Option." http://www.astronautical.org/pubs/vol40i3Feat.htm, February 28, 2002.
} 
The primary reason that this description of Aquarius is included in this thesis is because the author began her research by using Aquarius as a case study for new valuation techniques. In the process, it was discovered that in order to do a valuation of Aquarius, it was necessary to do a valuation of its potential markets. This led to the satellite servicing market analysis presented in Chapters 4 and 5. The satellite servicing analysis is presented from the perspective of using a low cost launch vehicle, such as Aquarius.

\subsection{Financial Valuation Tools}

This section introduces the concepts behind real options, considers the benefits and downfalls of other financial valuation tools, investigates different scenarios that yield themselves to being valued using real options, and illustrates how real options can be used to evaluate projects in the aerospace industry. This section also includes an example valuation, comparing net present value, decision tree analysis, and real options.

Some of the tools shown below, namely net present value/discounted cash flow and real options are used throughout the remainder of this thesis to value the satellite servicing market. The net present value/discounted cash flow approach is used in the satellite servicing analysis to capture the value of each case before accounting for flexibility. The real options approach is utilized to take into account the inherent flexibility in satellite servicing. The background for their use is presented here.

\subsubsection{What is the Real Options Approach?}

The real options approach is a financial valuation technique that uses the concepts behind financial option pricing theory (OPT) to value "real" (non-financial) assets. It is a tool that can be used to value projects that have "risky" or contingent future cash flows, as well as long-term projects; projects that are typically undervalued by standard valuation tools. An option is defined as the ability, but not the obligation, to exploit a future profitable opportunity.

Most projects have options embedded in them. These options give managers the chance to adapt and revise decisions based upon new information or developments. For example, if a project is determined to be an unprofitable venture for a company, the project can be abandoned. The option to abandon a project has value, especially when 
future investments are necessary to continue the project. The real options approach captures this value, along with the value of uncertainty in a project. Real options and option pricing theory will be used interchangeably throughout the remainder of the section.

\subsubsection{How Does Real Options Compare to Standard Valuation Techniques?}

\section{Traditional Net Present Value (NPV)}

NPV is a standard financial tool that compares the positive and negative cash flows for a project by using a discount rate to adjust future dollars to "current" dollars. The following equation can be used to calculate NPV.

$$
N P V=\sum_{i=1}^{N} \frac{C_{i}}{(1+r)^{i}}
$$

where $r$ is the discount rate, $C_{i}$ is the cash flow in period $i$, and $N$ is the total number of periods. The discount rate is determined by the expected rate of return in the capital markets and accounts for the "riskiness" of the project.

Two major deficiencies exist in this method. Managerial flexibility is ignored, and the choice of discount rates is very subjective. Managers often use inappropriately high discount rates to value projects (Dixit, 1994). In addition, NPV does not take into account the flexibility and influence of future actions inherent in most projects. Both using a high discount rate and ignoring the flexibility of using future "options" to make strategic decisions tend to lead to the under valuation of projects. However, one of the primary benefits of the NPV approach is that it is simple and understood by many people.

\section{Discounted Cash Flow (DCF)}

DCF is simply the sum of the present values of future cash flows. It has the same drawbacks as listed for NPV. It inherently assumes that an investor is passive. This means that once a project is started it will be completed without future strategic decisions based upon future information or outcomes. Thus, it typically leads to undervalued projects because it does not take into account the value of the options for future action. 
As with NPV, one its main benefits is its universal use. It is also adaptable to many types of projects.

\section{Decision Tree Analysis}

Decision analysis is a straightforward method of laying out future decisions and sources of uncertainty. It uses probability estimates of outcomes to determine the value of a project. By doing this, it is one of the few methods that takes into account managerial flexibility. The major downfall to this approach is that probability estimates are generally very subjective and as such are hard to form with much precision. The equations for this method are presented below in the example calculation.

\section{Simulation Analysis}

Simulation analysis lays out many possible paths for the uncertain variables in a project. Unfortunately, it is difficult to model decisions that occur before the final decision date using simulation analysis. This and the use of a subjective discount rate are the major drawbacks of this method of valuation.

\subsubsection{Where can the Real Options Approach be Utilized?}

The real options approach is a suitable method for valuing projects that:

- include contingent investment decisions

- have a large enough uncertainty that it is sensible to wait for more information before making a particular decision

- have a large enough uncertainty to make flexibility a significant source of value

- have future growth options

- have project updates and mid-course strategy corrections

As can be seen above, a real options analysis is not needed for all cases. Traditional methods of valuation correctly value businesses that consistently produce the same or slightly declining cash flow each year without further investment or follow-on opportunities. Real options are not necessary for projects with negligible levels of uncertainty. (Amram, 1999) 


\subsubsection{Where can Real Options be Utilized in the Aerospace Industry?}

The following are hypothetical examples used to illustrate the value of real options.

\section{Waiting-To-Invest Options}

BizJet, a company that produces business jets, is considering becoming the first to enter the supersonic business jet market. It has the option to start development today or to wait until the market outlook changes. Real options can capture the value of delaying this decision until the market uncertainty is resolved.

\section{Growth Options}

CallSat, a company that offers satellite cellular phone service, is considering entering the market in the populated areas of South America. This would require a significant investment. If this investment is made it would leave the option open to increase service in the future to the less populated areas of South America if the market proved to be worthwhile. A real options analysis of this project would include the value of the future option to increase service area.

\section{Flexibility Options}

Entertainment Sat is considering developing a constellation of satellites that provides either standard satellite television service or a new pay-per-view downloadable movie service. Instrument $\mathrm{A}$ is needed on the satellite to provide television service and Instrument $\mathrm{B}$ is needed to provide downloadable movie service. Instrument $\mathrm{C}$ is more expensive than both $\mathrm{A}$ and $\mathrm{B}$ but it allows the satellite to provide either television or movie service. Real options can be used to value the flexibility of Instrument $\mathrm{C}$, taking into account the fact that if one of the two markets proves to be less profitable than expected, or the opposite occurs, Instrument $\mathrm{C}$ has the ability to capture the most profitable market at any given time.

\section{Exit Options}

Sky ISP, a proposed satellite internet service provider, is interested in providing very fast internet connections throughout the US, using a constellation of satellites. Their fear is that the market is not large enough to support the substantial investment necessary to 
fund the development of satellites. Market forecasts look good today but what will they look like in a year when the satellites will be launched, requiring additional funding? Real options recognizes that the project can be abandoned if the market forecasts deteriorate. This option to abandon has value in that it limits the downside potential of a project.

\section{Learning Option}

StarSat is doing research on a new tracking instrument that will help satellites point more accurately towards their target. There are several different levels of accuracy foreseen as feasible, each requiring an additional investment. StartSat has the ability to stage its investments in order to capitalize on learning effects. If through developing the first tracker they gain knowledge about how to develop the next tracker, the future investment can be altered. The real options approach values the contingent decisions based upon the learning curve that StarSat faces.

\subsubsection{Valuations: Using the Binomial Real Options Approach}

This section will walk the reader through a simple example of valuation to illustrate the differences between net present value, decision tree analysis, and real options. The reader should take note of a few key points throughout the example. First, the NPV approach does not correctly value options because it assumes that once a project is started, it will be completed regardless of the outcome. Second, DTA and OPT valuations both take into account managerial flexibility, but do not result in the same answers. This is due to the way the two methods discount the value of options. DTA uses the same discount rate to discount the underlying project as well as the options. Since an option is always more risky than its underlying asset (Brealey and Myers, 1996), OPT valuations discount the option at a higher rate. This is more consistent with the theory that riskier cash flows should be discounted at a higher rate.

The valuation will be based upon the following scenario:

Sky ISP, as introduced previously, faces the following scenario. The market outlook for one year from now will either have high or low demand. If the demand for Internet connections is high, the market will be worth $\$ 800 \mathrm{M}$ and if the demand is 
low the market will be worth $\$ 200 \mathrm{M}$. The satellites will be launched in one year for a cost of $\$ 300 \mathrm{M}$. An initial investment of $\$ 250 \mathrm{M}$ must be made today in order to continue building the satellites needed to complete the system.

In financial market terms, the launch scenario corresponds to owning a call option on a stock with a price equal to the value (see calculation below) of the market and an exercise price of $\$ 300 \mathrm{M}$ (the cost of launching the satellites).

The market outcomes are illustrated in Figure 2-4.

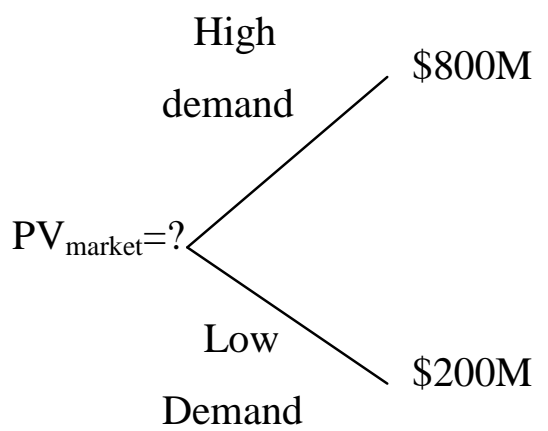

\section{Figure 2-4 Predicted Market Outcomes for Year 1}

The information needed in the analysis to follow is summarized here $(\$ M)$ :

- Initial investment today to continue building satellites, I:

- Present value of market without option to launch (calculation below), S: $\quad \$ 509$

- Future value of market with high demand, uS:

- Future value of market with low demand, dS:

- Probability of high demand, p:

- Probability of low demand, 1-p:

- Discount rate, $r$ :

- Exercise price, E:

- Maturity, t:

- Risk-free interest rate, $\mathrm{r}_{\mathrm{f}}$ :

In this example, a distinction will be made between the market and the project. The value of the market is defined as the amount of money a business would make if entering the 
market had zero costs associated with it. The value of the business/project is defined as the value of the market minus the cost of entering the market.(i.e. the exercise price of the option). In this case, the cost of entering the market is the cost of launch. The present value of the market is:

$$
P V=\frac{p(u S)+(1-p)(d S)}{(1+r)^{t}}=\frac{(0.6)(\$ 800)+(0.4)(\$ 200)}{1.1}=\$ 509
$$

\section{Net present value calculation}

The NPV of the business is found using the following formula.

$$
N P V=p \frac{u S-E}{1+r}+(1-p) \frac{d S-E}{1+r}-I
$$

This formula simply takes the value of the project in year 1 and discounts it back to year 0 . Using the assumptions above the net present value of the project is:

$$
N P V=0.6 \times \frac{\$ 800-\$ 300}{1.1}+0.4 \times \frac{\$ 200-\$ 300}{1.1}-\$ 250=-\$ 14
$$

The NPV valuation assumes that the option is exercised regardless of the market outcome. This is obviously flawed because a rational manager would not choose to launch the satellites if the demand were lower than the cost of launch. This leads to a negative NPV valuation.

\section{Decision tree analysis calculation}

Traditionally, this project would either not be undertaken because of its negative valuation, or a manager would go with his/her "gut" feeling that Sky ISP is a worthwhile project. Although this project is worthwhile as long as one considers the options (a.k.a. managerial flexibility) involved, it would be helpful to be able to quantify the manager's "gut" feeling. One method of remedying this is to use decision tree analysis. In finance terms, this method recognizes the manager's ability to not exercise the call option (i.e. launch the satellites) if the demand is low. This is illustrated below, where circles represent event nodes and squares represent decision nodes. The bold lettering indicates what decision a rational manager would make in the given situation. 


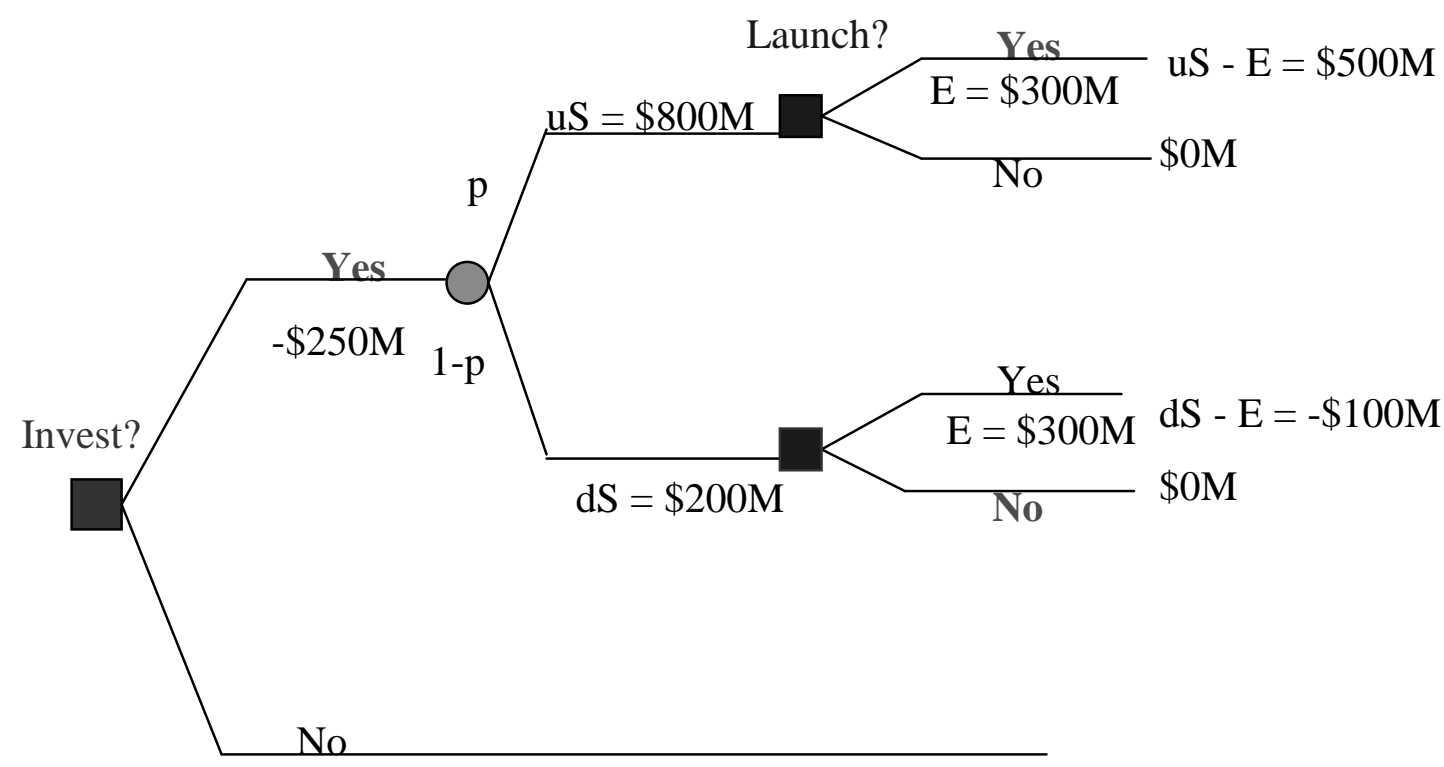

Figure 2-5: Decision Tree used for Decision Analysis and Real Options Valuation

The value of this project, according to decision analysis, is calculated using the following formula.

$$
V_{D T A}=\frac{p \times \max (u S-E, 0)+(1-p) \times \max (d S-E, 0)}{(1+r)^{t}}-I
$$

In this case, the decision tree analysis method gives:

$$
\begin{aligned}
V_{D T A} & =\frac{(0.6) \max (\$ 800-\$ 300,0)+(0.4) \times \max (\$ 200-\$ 300,0)}{1.1}-\$ 250 \\
& =\frac{0.6(\$ 500)+0.4(0)}{1.1}-\$ 250=\$ 23
\end{aligned}
$$

This valuation is significantly higher than the NPV approach because it assumes that the project would be abandoned if the launch costs exceeded the size of the market.

\section{Real options calculation}

The final approach covered here is real options. Using the binomial method (Brealey and Myers, 1996), there are two ways to approach this valuation. The one that will be used here is the risk-neutral approach. 
The risk-neutral approach is based on the surprising fact that the value of an option is independent of investors' preferences towards risk. Therefore, the value of the option in a risk-neutral world, where investors are indifferent to risk, equals the option's value in the real world.

If Sky ISP were indifferent to risk, the manager would be content if the business offered the risk-free rate of return of $5 \%$. The value of the market is either going to increase to $\$ 800$, a rise of $57 \%$, or decrease to $\$ 200$, a fall of $61 \%$.

$$
\underset{\text { Return }}{\text { Expected }}=\left(\begin{array}{c}
\text { Probability } \\
\text { of } \text { rise }^{\text {Pise }}
\end{array} \times 57 \%\right)+\left(\begin{array}{c}
\text { Probability } \\
\text { of }
\end{array}\right) \times(-61 \%)=5 \%
$$

This yields a probability of rise in the risk-neutral world of $56 \%$. The true probability of the market rising is $60 \%$. However, options are always riskier than their underlying asset (i.e. the project itself), which leads to the use of different probabilities for valuation. The use of risk-neutral probabilities effectively increases the discount rate used to value the option.

If there is low demand in the market, the market with the option to launch will be worth nothing. On the other hand, if the demand in the market is high, the manager will choose to launch and make $800-300=500$, or $\$ 500 \mathrm{M}$. Therefore, the expected future value of the market with the option is

$$
\left(\begin{array}{c}
\text { Probability } \\
\text { of } \quad \text { rise }
\end{array}\right) \times 500+\left(1-\begin{array}{c}
\text { Probability } \\
\text { of }
\end{array}\right) \times 0=(.56 \times 500)+(.44 \times 0)=\$ 280
$$

Still assuming a risk-neutral world, the future value is discounted at the risk-free rate to find the current value of the project with the option to launch as

$$
\frac{\text { Expected future value }}{1+r_{f}}-I=\frac{280}{1.05}-250=\$ 16
$$

The value of the option to launch is the difference between the value of the business with the option (the OPT valuation) and the value of the business without the option (i.e. the NPV). 


$$
\begin{gathered}
\begin{array}{c}
\text { Value of business } \\
\text { with launch } \\
\text { option }
\end{array} \\
=\$ 16 M-(-\$ 14 M)=\$ 30 M
\end{gathered}
$$

\section{Multiple option example}

Although single options are often very important to analyze, as they may be all that a business faces, multiple or compound options are generally more interesting. Adding another option to the one discussed above produces interesting results. Assume that oneyear after the launch decision is made, a new satellite data transfer market emerges. This market has a $30 \%$ chance of being worth $\$ 400 \mathrm{M}$ and a $70 \%$ of being worth $\$ 50$. The operations and marketing costs of entering this new market amount to $\$ 100 \mathrm{M}$. The situation is illustrated graphically in the figure below, where the probabilities shown are the probabilities used in the NPV and decision analysis valuations. The risk-neutral probabilities, used in OPT, are not shown. 


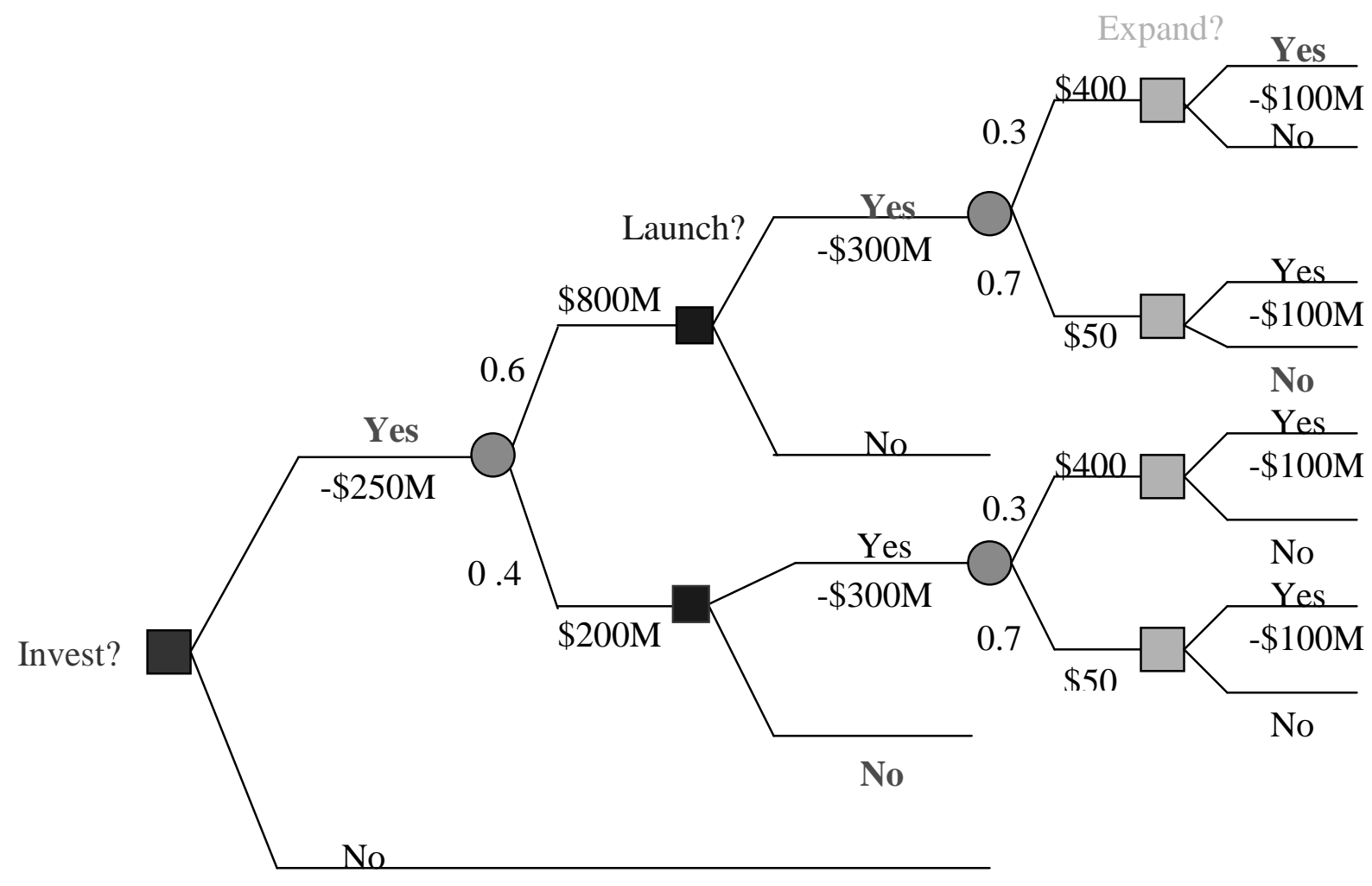

Figure 2-6 Example of Multiple Options

Although the calculations will not be covered in detail here, the NPV, DTA, and OPT valuations are listed below for various cases. In the analysis the following are used:

- $\mathrm{uS}_{1}$ : Future value of original market with high demand

- $\mathrm{dS}_{1}$ : Future value of original market with low demand

- $\mathrm{E}_{1}$ : Exercise price of option to launch (cost of launch)

- $\mathrm{uS}_{2}$ : Future value of new data transfer market with high demand

- $\mathrm{dS}_{2}$ : Future value of new data transfer market with low demand

- $\mathrm{E}_{2}$ : Exercise price of option to expand (cost of expansion)

- $\mathrm{r}_{1}$ : Discount rate for launch option $=10 \%$

- $\mathrm{r}_{2}$ : Discount rate for option to expand $=15 \%$

- $\mathrm{r}_{\mathrm{r}}$ : Risk-free interest rate $=5 \%$

All numbers below are in \$M. 
Table 2-1 Various Cases of Two Option Valuation

\begin{tabular}{|c|c|c|c|c|c|c|c|c|c|c|c|}
\hline $\begin{array}{c}\text { Case } \\
\text { number }\end{array}$ & $\mathrm{uS}_{1}$ & $\mathrm{dS}_{1}$ & $\mathrm{E}_{1}$ & $\mathrm{uS}_{2}$ & $\mathrm{dS}_{2}$ & $\mathrm{E}_{2}$ & $\mathrm{NPV}$ & $\mathrm{DTA}$ & $\mathrm{OPT}$ & $\begin{array}{c}\text { Option to } \\
\text { launch }\end{array}$ & $\begin{array}{c}\text { Option to } \\
\text { expand }\end{array}$ \\
\hline $\mathbf{1}$ & $\mathbf{8 0 0}$ & $\mathbf{2 0 0}$ & $\mathbf{3 0 0}$ & $\mathbf{4 0 0}$ & $\mathbf{5 0}$ & $\mathbf{1 0 0}$ & $\mathbf{3 0}$ & $\mathbf{6 5}$ & $\mathbf{5 2}$ & $\mathbf{2 2}$ & $\mathbf{7 5}$ \\
$\mathbf{2}$ & $\mathbf{8 0 0}$ & $\mathbf{2 0 0}$ & $\mathbf{3 0 0}$ & $\mathbf{6 0 0}$ & $\mathbf{5 0}$ & 100 & $\mathbf{7 7}$ & 105 & 89 & 11 & 127 \\
3 & $\mathbf{8 0 0}$ & $\mathbf{5 0}$ & 300 & 400 & 50 & 100 & -25 & 65 & 59 & 84 & 75 \\
4 & 800 & 200 & $\mathbf{4 0 0}$ & 400 & 50 & 100 & -61 & 11 & 0 & 61 & 75 \\
5 & 800 & 200 & 300 & 400 & 50 & $\mathbf{2 0 0}$ & -14 & 51 & 40 & 54 & 50 \\
\hline
\end{tabular}

Case 1 is the baseline case for the rest of the analysis. It illustrates how the addition of the option to expand significantly increases the value of the project.

Case 2 illustrates the effect of increasing the upside potential of the option to expand. As can be seen above, it significantly increases the value of the project. It also makes the option to launch worth less because even if the initial demand were low, the option to expand makes it worthwhile to launch the satellites.

Case 3 illustrates the effect of decreasing the future value of the original market with low demand. In this case, the value of the option to launch increases because one can choose not to launch if the demand were low. As expected, the DTA value of the project does not change because the manager would only launch if the demand were high.

Case 4 illustrates the effect of increasing the exercise price of the option to launch (i.e. increasing the launch costs). The NPV valuation becomes negative, while the OPT valuation goes to zero. The reason that the DTA valuation remains positive is due to the way in which discounting takes place.

Case 5 illustrates the effect of increasing the exercise price of the option to expand (i.e. increasing the cost of entering the new data transfer market). The NPV is much more negative than the DTA or OPT valuations because it does not correctly value options. In 
addition, this case is a good example of the DTA valuation being greater than the OPT valuation. This is due to the different ways that DTA and OPT treat discount rates.

\subsubsection{Extension to the Black-Scholes Formula}

Thus far, all quantitative discussion of real options in this thesis is based upon the binomial method. This is a simplified version of option pricing theory that assumes that there are only two possible outcomes for a project. Although this method can be used to value options over short time periods or in very special cases where only two outcomes are possible, it is often unrealistic.

One means of solving this issue is to break the total time period into smaller intervals. For an example of this refer to Brealey and Myers, 1996. As the time interval period used for each option shortens, the valuation becomes more realistic because more outcomes are possible. Ideally one would keep shortening the interval periods until eventually the stock price (or project value) varies continuously. This leads to a continuum of possible outcomes. Fortunately, this is exactly what the Black-Scholes formula, which the authors were awarded the 1997 Nobel Prize in Economics for, does. The formula is

$$
V_{\text {OPTION }}=P \times N\left(d_{1}\right)-P V(E) \times N\left(d_{2}\right)
$$

where

$$
\left\{\begin{array}{l}
N(x)=\int \frac{1}{\sqrt{2 \pi}} e^{-y^{2}} d y \\
d_{1}=\frac{\left[\ln (P / E)+\left(r+\sigma^{2} / 2\right) \times t\right]}{\sigma \sqrt{t}} \quad N \text { : cumulative normal distribution function } \\
d_{2}=d_{1}-\sigma \sqrt{t}
\end{array}\right.
$$

and

$\mathrm{P} \quad=$ share price (value of project) 
$\mathrm{r} \quad=$ risk-free interest rate

$\mathrm{PV}(\mathrm{E})=$ present value of exercise price of option (discounted using risk-free rate)

$\mathrm{t}=$ number of periods to exercise date

$\sigma \quad=$ volatility of the share price per period of rate return (continuously compounded) on stock

In addition to accounting for the fact that projects generally have a continuum of possible outcomes, the Black-Scholes formula does not require an arbitrary discount rate. Although the binomial method does not technically use a discount rate, using a discount rate is almost always inevitable to determine the present value of the price of the stock (i.e. project).

\subsection{Satellite Servicing}

Satellite servicing represents a paradigm shift in the way space systems are currently designed and maintained. This section presents an overview of the history of servicing, including actual on-orbit servicing of space hardware as well as previous research. In addition, a brief introduction to a current satellite servicing program is presented.

\subsubsection{History of Servicing}

The idea of using another spacecraft to aid in performing maneuvers, maintenance and upgrade operations seems quite revolutionary. However, there are many instances of onorbit upgrades and maintenance being performed. These include Skylab, the Russian Space Stations Program, and the International Space Station. Although these are all manned missions, they represent a desire to maintain and upgrade space hardware on orbit.

\section{Servicing of unmanned spacecraft}

The first unmanned spacecraft to be serviced on orbit was the Solar Maxim Mission (Waltz, 1993). The spacecraft was serviced by the Challenger Shuttle astronauts because it was deemed more cost-effective to do so than to build a replacement spacecraft. Since 
then, Shuttle astronauts have performed several unanticipated maintenance missions (Lamassoure, 2001).

The most well known example is the servicing missions performed on the Hubble Space Telescope (HST). The first servicing mission, in 1993, was a repair mission to correct for a flawed mirror. The second servicing mission, in 1997, served as an upgrade mission by installing new instruments. The third servicing mission, in December 2001, upgraded components and replaced failing gyroscopes. The fourth servicing (originally planned as part of the third servicing mission), in March 2002, installed a new camera, for increased imaging capability, and performed other needed upgrades. The fifth servicing of HST is scheduled for 2004.

Although each of the missions above required the use of astronauts, as opposed to another spacecraft, they serve to illustrate the mindset of on-orbit servicing. For more information on these servicing missions, as well as others, see Lamassoure, 2001.

\section{Unmanned servicing of unmanned spacecraft}

Although it was deemed to be cost-effective to use astronauts to service both the SMM spacecraft and HST, most satellites would be less expensive to replace than the cost of a manned servicing mission. Thus, to become a more viable option, servicing needs to be performed by an unmanned spacecraft. Several studies have been performed to examine the feasibility and cost-effectiveness of this type of satellite servicing.

\section{Studies on on-orbit upgrading}

The Space Assembly, Maintenance, and Servicing (SAMS) project was funded by the Department of Defense, Strategic Defense Initiative Office, and NASA to determine costeffective SAMS capabilities to meet requirements for improving space systems capability, flexibility, and affordability.

The Spacecraft Modular Architecture Design (SMARD) study examined the serviceability of currently designed spacecraft, suggested alternatives to the design to enable servicing, and examined the cost-effectiveness of particular servicing cases (Davinic, 1997). 
Upgrading the GPS constellation using autonomous on-orbit servicing has also been studied. One of the studies considered necessary structural modifications to the GPS satellites to enable servicing (Hall, 1999). The other performed a trade study to determine the best servicing architecture (Leisman, 1999).

The SAMS, SMARD, and GPS studies researched the cost-effectiveness of servicing from the perspective of using upgrades or functional replacements to avoid satellite replacement. For a top-level description of each of these studies, see Lamassoure, 2001 and Saleh, 2001.

Studies on value of flexibility of on-orbit servicing

One of the many benefits servicing provides is flexibility to adapt to future needs. This flexibility has significant value. Two studies have been performed in this area to propose frameworks for valuing flexibility. One of the primary contributions of both of these studies is their focus on the potential servicing client and how the client values servicing, as opposed to how much the servicing architecture costs.

The first examines the value of flexibility as it relates to both commercial and military systems. The commercial valuation of flexibility deals primarily with the option for life extension using upgrades on orbit. The military valuation deals primarily with options to relocate satellites as an alternative to global coverage (Lamassoure, 2001).

The second study suggests an evaluation process to account for the flexibility inherent in on-orbit servicing. This real options-based framework examines the value of flexibility for satellite life extension and relocation (Saleh, 2001).

\subsubsection{Current servicing program: Orbital Express}

As the U.S. government is increasingly recognizing the potential advantages offered by on-orbit servicing, the Defense Advanced Research Projects Agency (DARPA) is currently sponsoring the Orbital Express Space Operations Architecture, whose goal is to study and demonstrate autonomous techniques for on-orbit servicing. The program intends to develop and demonstrate techniques for autonomous on-orbit refueling and reconfiguration. 
To demonstrate these techniques, an Autonomous Space Transporter and Robotics Orbiter (ASTRO) servicing spacecraft will be used to conduct docking, refueling, and pre-planned product improvement ("P3I") operations. In addition to the servicing spacecraft itself, studies are being performed for the development of on-orbit storage and handling of liquid and/or gaseous consumables ${ }^{5}$.

\footnotetext{
${ }^{5}$ For more information on Orbital Express see http://www.darpa.mil/tto/programs/astro.html.
} 


\section{Chapter 3. Valuation: Interface Between Technology and Economics}

\subsection{Introduction}

This chapter serves to introduce a new valuation framework, which accounts for the economics, technology, and flexibility associated with a project. First, a discussion of why a new valuation technique is important is presented. Next, the new valuation framework is discussed. Finally, the steps to apply the framework for valuation is presented.

\subsection{Why are new valuation techniques necessary?}

Current valuations fail in three major respects. First, they do not generally aid in the process of determining what the best product is from the perspective of providing value to the client and the provider. Second, they lack the ability to take into account both the economic and technological aspects of a project. Third, they neglect to quantitatively account for flexibility in a project. Each of these failures will be discussed below.

\subsubsection{Doing the Right Job}

The Lean Aerospace Initiative (LAI) is dedicated to improving the practices of the aerospace industry. The Product Development team, in particular, focuses on the "fuzzy" 
front-end development of a project. One of the fundamental principles the team uses is "Doing the right job and doing the job right."

Frequently on projects, there is a strong focus on doing the job right. That may be by applying lean principles or by other means particular to a group or company. However, "doing the job right" means nothing if the program is doing the wrong job. For instance, a program can be internally lean and well managed, but if the program is producing a product for which there is little or no market, it does not matter how well it is run.

Current valuations in aerospace tend to focus on valuing a point-designed product. This does not necessarily lead to doing the "right job." Instead it may lead to doing a reasonable job, but not necessarily the best one. An important part of a good valuation is to aid in determining what the "right job" is. The "right job" is the one that provides the most value to the client and provider. Generally, these two things are highly correlated. The more value the provider provides to her client, the more the client is willing to pay, thus providing value for the provider.

\subsubsection{Economics and Technology in Valuations}

One of the fundamental drawbacks of current valuation techniques is that they do not properly account for both economic and technical aspects associated with a given project. To understand why this is true it is important to understand the background of the people who perform these valuations.

\section{Engineers}

Technological products are developed by engineers. As a stakeholder in the project, an engineer's primary goal is generally to solve a technical problem. This can lead to several difficulties in the process of valuing a project. First, engineers generally lack an understanding of economics and the potential market for their product. Second, engineers can get wrapped up in the technical aspects of their project and tend to neglect the dynamics of its potential market value by assuming a market for their product exists.

\section{Finance/Marketing}

The group of people that are typically the most involved with financial project valuation are the finance and marketing employees of a firm. Although this group may have a 
good understanding of economics and markets, they lack technical understanding of the project. This can lead to several issues. First, they may promise a client something that is technologically unfeasible; given the amount the client is willing to pay for the product. Second, there may be a way to adapt certain technologies to a market, but without a solid technical background it is difficult to understand the best approach to adapting current or future technologies to meet the needs of a given market.

\section{Combining the two groups}

Although the most successful valuations contain inputs from both engineering and finance groups, combining these two groups is not trivial. The problem here lies in that the two groups often speak different languages. The best way to solve this issue is to include people in the valuation who have a solid technical and business background. This provides an interface between the two groups, which will tend to produce more accurate valuations.

\subsubsection{Valuing Flexibility}

As discussed in Section 2.3.4, there are often options associated with projects. In current valuations, these options are often overlooked.

In fact, strategically important projects often fail internal financial tests. Analysts, in a quest to justify their "gut feel," tend to manipulate the evaluation process, raising cash flow forecasts to unlikely levels. Key managers make decision colored by optimism and bounded by their degree of risk aversion (Amram, 1999).

Current valuation techniques often lead to the wrong answer and generally undervalue technologies because they lack the ability to value flexibility (Boer, 1998). Flexibility has value, especially in situations with high uncertainty.

One means of valuing flexibility is to use real options. For a complete discussion of the types of flexibility imbedded in projects and how to use real options to value them, see Section 2.3. For a complete discussion on the merits of flexibility as it applies to aerospace products, see (Saleh, 2001). 


\subsection{Valuation Framework}

A new valuation framework is presented to address the issues of doing the right job, accounting for economic and technical aspects, and the value of flexibility in a potential project. The three primary aspects of the framework are economics, technology, and the interface between them. Flexibility and determining the right job are also included in the framework. A visual representation of the framework is presented in Figure 3-1. 


\section{Economics}

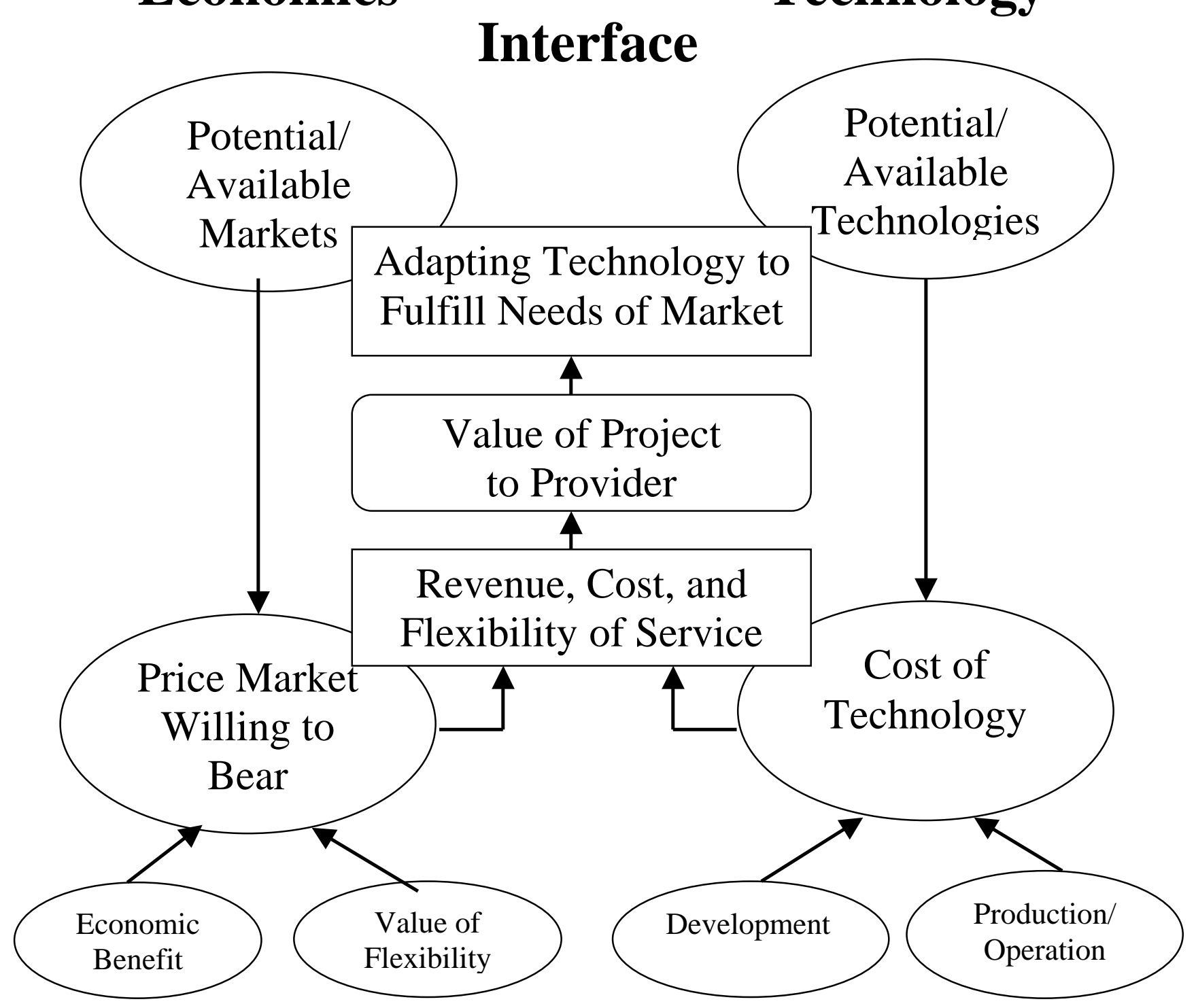

Figure 3-1 Valuation Framework

Each of the aspects of the framework is discussed below.

\subsubsection{Economics}

When a space system is developed, it is easy to get consumed with the technology and forget that the system must provide value to a potential client for the client to be willing 
to pay for it. After the conceptual idea for a service/product is developed, it is important to look at the idea from the perspective of a potential client to determine not only what the service should be, but also what the potential client is willing to pay for it.

The left side of Figure 3-1 demonstrates some of these important aspects of a valuation. Often, similar kinds of service can be provided using different approaches, with each of these approaches providing a different value to the potential client. The various means of providing service should be investigated to determine how much value each approach provides to the potential client.

The reason the value provided to the client is important is because this value determines the price they are willing to pay for the service and also gives an indication of which is the best approach to providing the service. Although there are many other drivers of the price the market will bear, the primary drivers of this price are the direct economic benefits the service provides the client and the value of flexibility the service provides.

Projects often fail because incorrect assumptions are made about potential markets. These include assuming that the market is willing to pay the cost of the service plus some profit margin and assuming that the current market is static, in that there will not be any new competition or other technology developments. These assumptions can lead to large losses for the provider.

It is certainly possible that the service may not be worth the cost plus profit margin of the system to the potential client. In this case, the provider will receive little to no revenues and will not cover the costs of the system, much less generate a profit. The possibility of not having any or enough clients should provide adequate incentive to examine the benefits of the service to the potential client to determine what value it provides them.

It is also possible that there are other means of providing service for the potential market, which result in competition. It is crucial to realize this when considering the price the market is willing to pay for the potential service. If the competition can provide equivalent or greater value for the same or lower price, then it would be very difficult to provide a valuable service the market. 


\subsubsection{Technology}

Most aerospace companies are very familiar with the technology side of the framework. Solving technical problems is what engineers do best. However, it is important to realize that in depth technical analysis is expensive and should not be undertaken with the assumption that a market for the technology will exist. Instead, the knowledge from preliminary studies of the technology should be used to estimate costs and aid in determining the economic and technical feasibility of meeting the market's needs before a detailed technical analysis is undertaken.

It is important to determine the primary technical risks in design and on-orbit operations for each of the possible approaches to providing service. The risks of design are important to understand, as they can significantly impact the development costs and schedule. The technical on-orbit risks are important to understand because they impact the system design, in that unless a particular approach is highly valuable to the potential client, the client will be unlikely to purchase a service or product that is high-risk. The risks on orbit can also have a significant impact on operations costs.

\subsubsection{Interface}

Although the economics and technology aspects of the framework are extremely important, the interface between the two is where the crucial information about the feasibility of the market is determined. The economics side of the framework provides the potential client's value for each of the potential service approaches. The technology side provides the costs and risk assessments for each of the potential approaches. The interface provides information on which service approach is most feasible by determining the value each approach provides to the potential service provider and thus, how to best adapt technologies to meet the needs of the market.

The interface between economics and technology uses the revenue and cost from the economics and technology sides, respectively, along with the value of flexibility, to determine the value of each service approach to the provider. This leads to the proper adapting of technology to meet the needs of the market. The approach chosen many not be the best from the perspective of the potential client, because the client's "best" service 
may be too technically difficult or too expensive for the provider to provide. By the same token, the approach chosen may not be the least expensive for the provider to provide. Instead, it will be the best mix between the two stakeholders; resulting in a space system that provides value to both the potential client and provider.

\subsection{Applying the Valuation Framework}

The framework presented in Section 3.3 is a representation of the interface between economics and technology, as it applies to valuations. To demonstrate using this framework for valuations, the process is broken into steps. These steps are also the goals of the valuation in that each step not only gains information that is necessary to complete the overall valuation, but also gives other insights along the way. The goals of the valuation are to determine:

- If there is a potential market

- What the market wants

- The value of the service/product to potential clients

- How much will it cost for someone to provide service/product to meet market needs

- The value of the project to the provider

The valuation framework applies to both services and products. For example, it can be used to value the satellite servicing market, as well as to value the production a new satellite configuration. For the sake of brevity, "service" is used in place of "service/product" throughout the remainder of the chapter.

In the process of performing a valuation using this framework, it is necessary to perform technical/economic trade studies to most effectively meet the goals of the valuation. One of the primary benefits of the valuation is the technical and economic information gained in the process of performing these trades. This, along with each of the steps in the valuation is discussed throughout the remainder of Section 3.3. 


\subsubsection{Is there a potential market?}

The first step in the valuation process is to determine if there is a potential market. It is necessary to consider several things to do this.

- What is currently available to the potential client?

- How much value does the current service provide to the client?

- What is the current competition? What value do they provide?

- Are there any possible competitors in the future that can provide equivalent or greater value to the client?

- What are the different possible services that can be provided to enhance the value to the potential client?

The information gained from the questions above fall nicely into a trade study, with each case representing a different means of providing service including the current services, future competition, and potential services. The purpose of the trade study is to determine if the potential service is more valuable to the client than the current or possible competition services. The trade study should take into account the technical and economic implications of each of the potential services in question. It should also include the valuation of flexibility (i.e. options) that each service provides. The key is to focus on the client and the value provided to them by each service. The cost of the service itself need not be taken into account, as that will be determined and accounted for later in the valuation. However, the cost of the client's assets, i.e. her satellite, should be accounted for.

Performing a trade study to explore the market viability, from the perspective of the client, requires the use of both technical and economic inputs. Although some of the inputs may significantly impact the outputs of the study, many of them may not. It is important to recognize the difference between inputs that affect the answer and those that do not. Finding accurate inputs can often be time consuming and resources intensive. Thus, knowing which inputs must be very accurate to produce accurate results is very important. Performing a sensitivity analysis on the trades model can be helpful in 
determining what level of accuracy for each input is necessary. This applies to the inputs for all trades performed throughout the valuation process.

\subsubsection{What does the market want?}

Using the information from the trade study discussed above, it is possible to gain a good understanding of what the market wants. This is based on what provides the most value to the potential client. As this was one of the purposes of the trade study, this information is readily available. It is important, at this stage in the valuation, to not focus solely on one potential service (unless there are no alternatives). As the costs of providing each of the potential services have not been calculated, it is not clear if it is possible to provide a particular service even if it provides the most value to the potential client. Therefore, alternatives must be examined as they may have different cost characteristics.

Determining what the market does not want is just as important as determining what the market wants. If a potential service provides less value to the client than existing services or potential competition, then this service will not provide additional value to the market. Thus, it can be dismissed and the work of continuing the valuation of that particular service can be avoided.

\subsubsection{What is the value of the service to the client?}

The purpose of this part of the valuation is to determine how much a potential client is willing to pay, regardless of how much it costs, and how large the market is for each potential service. This implies the revenue stream of the potential service provider.

The amount that a potential client is willing to pay for a service is dependent upon the value it provides them. If the service is a replacement for current services or products, then the price they are willing to pay is a percentage of the value that it provides them. If the service is in addition to current services or products its value is simply the additional value it provides over the current service.

As the client's value for the service is already estimated in the trade studies, estimating what the client is willing to pay is simply a matter of comparing the service to current and 
competition services and estimating what percentage of the client's value for the service they are willing to pass on to the service provider. Although this percentage may be hard to estimate, a sensitivity analysis to this number can be utilized to see how it affects the final provider value.

In addition to the price the client is willing to pay, one needs to know how many clients there are to determine the revenue stream. Determining the market size requires making an estimation of potential clients. In the case of space systems, there are many available forecasts for launches and satellites (Commercial Space Transportation Forecasts, 2001).

\subsubsection{How much will it cost to provide service?}

After the revenue stream for the provider for each potential service is determined, the costs of each potential service must be examined to see if the service costs less to provide than the price the client is willing to pay. The costs should include development costs and production or operating costs (depending on whether it is a product or service that is being provided).

\subsubsection{What is the value of the project to the provider?}

Now that the revenues and costs are known, finding the value of providing the service is a matter of using these numbers and accounting for flexibility in the project. ${ }^{6}$ Just as determining the value to the client forces the use of trade studies to determine the best way of serving the client, determining the value to the provider of providing the service forces the use of trades.

This is the final part of the valuation and determines if the market is viable. If the servicing provider's total present value for providing the service is negative, they will choose not to provide the service.

\footnotetext{
${ }^{6}$ For a more in depth discussion of valuing flexibility and the different types of flexibility or options available see Section 2.3.
} 


\subsubsection{Learning by applying the valuation framework}

In the course of applying the framework and attempting to predict the value of a particular project, one will inevitably gain insights into many of the interactions between the economics and technology, client and provider values, and design trades and flexibility. Equally as important, if not more so, as the final "answer" in the valuation model is the information gained during the process.

Trying to value a project is like climbing a mountain. Although getting to the top, i.e. coming up with an "answer", is important, the climbing process and lessons learned along the way are equally, if not more, important.

In the case of space systems, many important insights are gained during the valuation process. For example, people's perception of the risk involved with developing and operating a novel system are more clearly understood after the valuation process is performed. This information is very useful in determining how much effort (marketing, studies, etc.) is necessary to convince a potential client of the technical feasibility of the project.

The primary objective of a climb is generally to reach the summit. There are many reasons for this; one of which is to see the view. The view from the summit depends on where the climber looks and who the climber is. There may not even be a view, depending on the weather. If the view is not clear, does that mean that the climb is pointless? Of course not. The climber had to go through essentially the same process of getting to the summit, as she would have on a day with a beautiful view.

Similarly, when performing a valuation, the goal is often to get "the" answer. Just as there are many different views from the top of a mountain, there are many different ways of interpreting a valuation. What one sees is generally dependent on who one is. In addition, the answer may not be as clear as one would like it to be. Is all lost? No. The information gained along the way exists regardless of the clarity of the answer.

Although it is very exciting (and generally exhausting) to reach the summit of a mountain, all good climbers recognize that summiting is not the end of a climb. The climber must be able to use the information gained on the ascent to guide her down the 
mountain. In a valuation, getting the answer is not the end of the process either. One must interpret the results and perform sensitivity analysis to determine the relative significance of particular inputs.

\subsubsection{Valuation Framework Flowchart}

The valuation framework presented above is used throughout the remainder of this thesis to evaluate the satellite servicing market. Much of the previous research in the field of on-orbit servicing focuses on the cost benefit of specific types of servicing (Davinic, 1997) and (Leisman, 1999). Although this is an important aspect of the economic picture, it is certainly not the entire picture. The cost benefit approach is solely the supply-side. The demand-side must be examined as well, to determine if there is a potential servicing market. Figure 3-2 shows a scenario in which the demand for servicing is lower than the cost of servicing, which makes the market unviable.

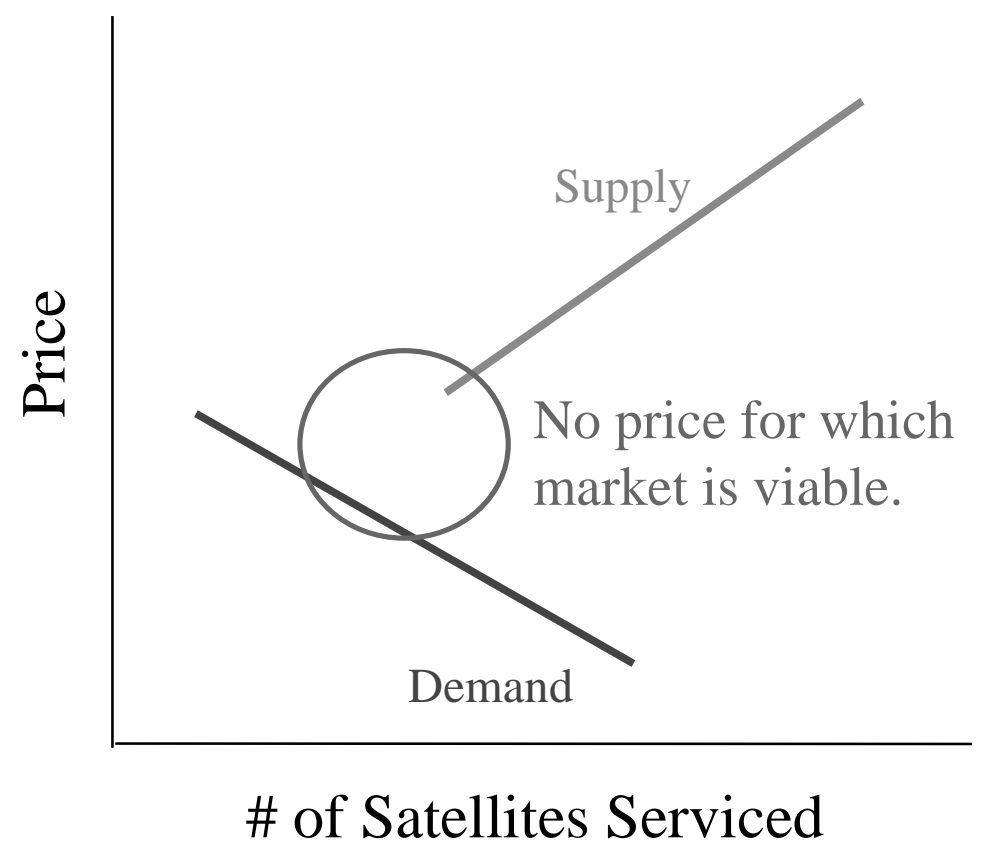

Figure 3-2 Example of Scenario Where Servicing Market is Not Feasible. 
This is only one example of how the supply and demand curves do not cross. It is not meant to be a representation of the actual supply and demand curves for the servicing market. Rather, it is presented to illustrate that one must examine the demand of a market, as well, to determine its viability.

The flow of the valuation analysis is presented in Figure 3-3. Its setup is derived from both the valuation framework and the need to examine the project from the prospective of both the client and provider. 


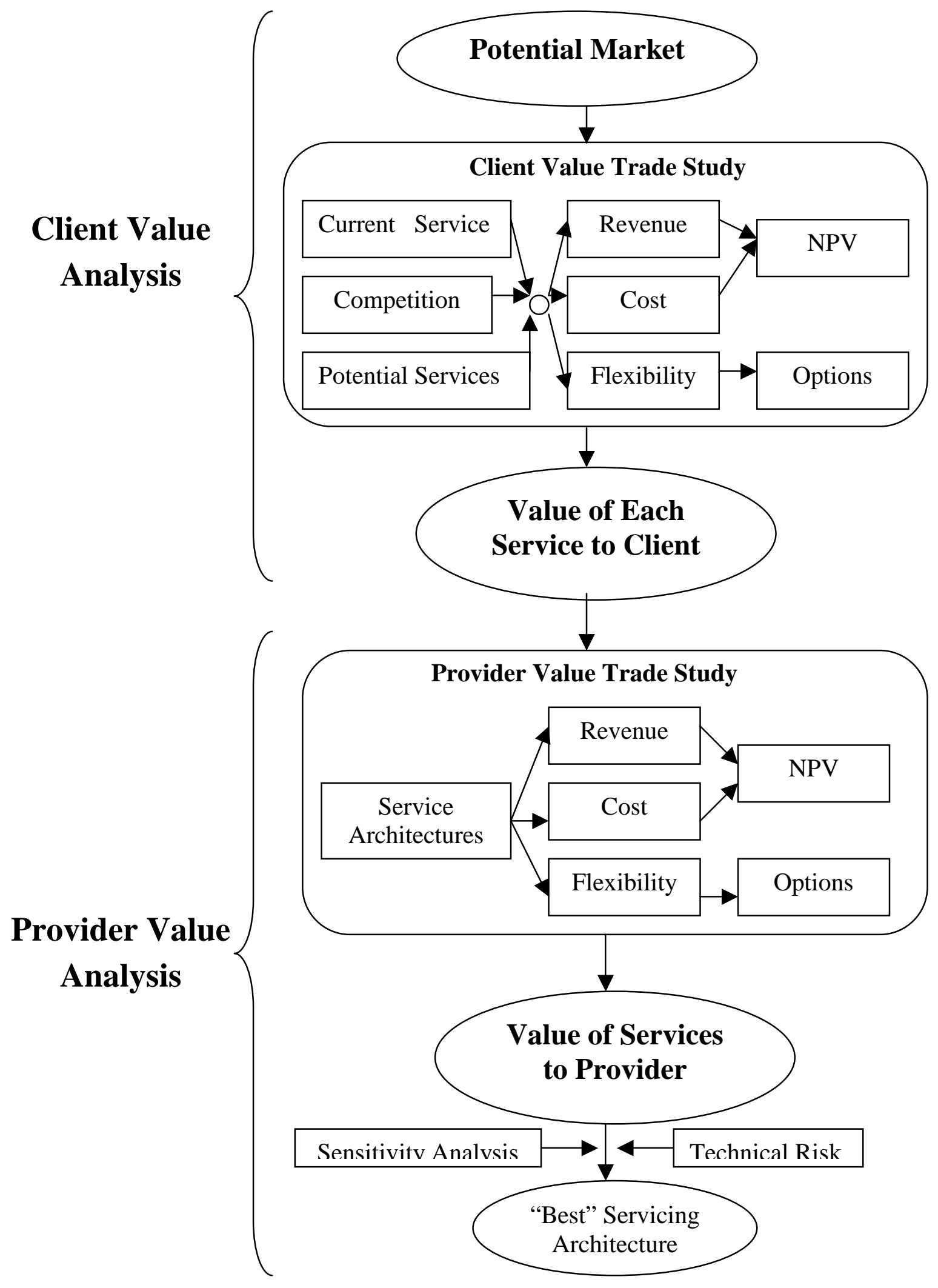

Figure 3-3 Flowchart for Valuations 
Chapter 4 presents the client value analysis by analyzing the current, future competition, and potential services to determine the value to the client of each case. Chapter 5 presents the provider value analysis by using the information gained in Chapter 4 to investigate the necessary architecture and value of each potential service.

\subsection{Conclusions}

A new valuation technique is necessary because current techniques neglect to address many important aspects of a technical project. First, they do not generally take into account both the economics and technical aspects of a project by considering both the potential client and provider perspectives. Second, they do not quantitatively consider the value of flexibility. Third, by neglecting the first two aspects, valuations do not aid in the determination of the "right" service approach. Part of the reason that many current valuations are flawed is because of the lack of understanding that engineers tend to have of economics and that finance/marketing employees tend to have of technology.

This chapter introduced a new valuation framework, which uses the economic, technical, and flexibility aspects of a project to determine the best means of adapting technology to meet the needs of a market. The framework stresses the necessity of examining a potential market from the perspective of both the potential client and the provider.

By examining the market from the perspective of the two primary stakeholders, the client and the provider, it is possible to determine the best service approach to provide the greatest value for both the potential client and the provider. An additional bonus to performing the valuation using the framework presented in this chapter is that it forces the use of trade studies, which provides many opportunities for learning about the technological, economic, and flexibility aspects of a project, as well as the interface between these three. 


\section{Chapter 4. Satellite Servicing: Client Value Analysis}

\subsection{Introduction}

A satellite servicing analysis is presented in this chapter. The primary purpose of this chapter is to establish the viability, or lack thereof, of the commercial GEO satellite servicing/refueling market from the client's perspective. The chapter begins with the purpose of the study, and then moves on to the overall structure of the analysis. The analysis is broken down into several cases, each examining the viability of a different servicing configuration. Each of the servicing cases is presented individually. Finally, the cases are presented together and each of the cases is discussed in terms of its technical risk.

\subsection{Purpose}

The client value analysis, when completed, serves as the primary input for the provider value analysis, which in this instance is the valuation of the satellite servicing market. In order to determine the value of this market, it is first necessary to determine what the client gains economically by using servicing. This drives the price the client, henceforth referred to as the client, is willing to pay for servicing and the size of the market. If the economic benefit to the client is large, it is likely that more clients will use servicing. If 
the economic benefit is small, it is likely that a smaller number of clients will be willing to undertake the added risk of servicing.

A thorough analysis of the economic benefit of servicing to the client must include the full spectrum of possible alternatives - that is, different means of servicing and the corresponding economic payoffs to the client.

For the purpose of this analysis, servicing is defined as the replenishment of consumables (refueling) and/or the use of another spacecraft to aid in performing maneuvers.

The purpose of this analysis is to determine:

- The economic benefit to a client of having his/her satellite serviced using several servicing approaches

- The highest-value servicing approach for the client

By fulfilling the goals above, the analysis de-couples the costs and benefits of servicing to determine if there is any economic benefit to clients of having their satellites serviced. If there is not, then no commercial market for servicing exists. If, on the other hand, there is an economic benefit to the client, that benefit is quantified. Thus, the client value analysis determines the viability of the commercial GEO satellite servicing market.

All other factors being equal, a satellite owner, henceforth called the client, would be willing to pay a percentage of the benefit they gain from servicing to the servicing provider. Thus, once the benefit to the client is determined, an estimate of how much she is willing to pay to have her satellite serviced can be found. This estimate can then be compared to the cost if servicing to see if an economically viable price exists. It can also be used as a cost constraint measure for design of the designated servicing architecture. The provider's perspective and costs of servicing are presented in the next chapter.

\subsection{Approach}

When determining the economic viability of a technical market, it is important to recognize that the economic and technical aspects of the project are not mutually exclusive. This analysis takes into account both of these aspects by examining the major spacecraft configuration changes associated with each servicing approach and tying it to 
their respective financial rewards and penalties. The analysis also includes a description of the technical risk associated with each case.

It is also important to trade proposed systems against current systems and possible future competition. Thus, this analysis examines both satellites that use servicing and satellites that do not. The satellite servicing cases are labeled AQR \#, where \# indicates the case number. The satellites that do not use servicing include the baseline satellite case Program A - and possible sources of competition, labeled Comp \#. The baseline, AQR, and Comp cases are traded against each other. The final trade is based upon economic benefit provided to the client and the consideration of risk.

An underlying assumption to this analysis is that the client's goal is to maximize profits. In the simplest form, profits are a function of revenue and expenses.

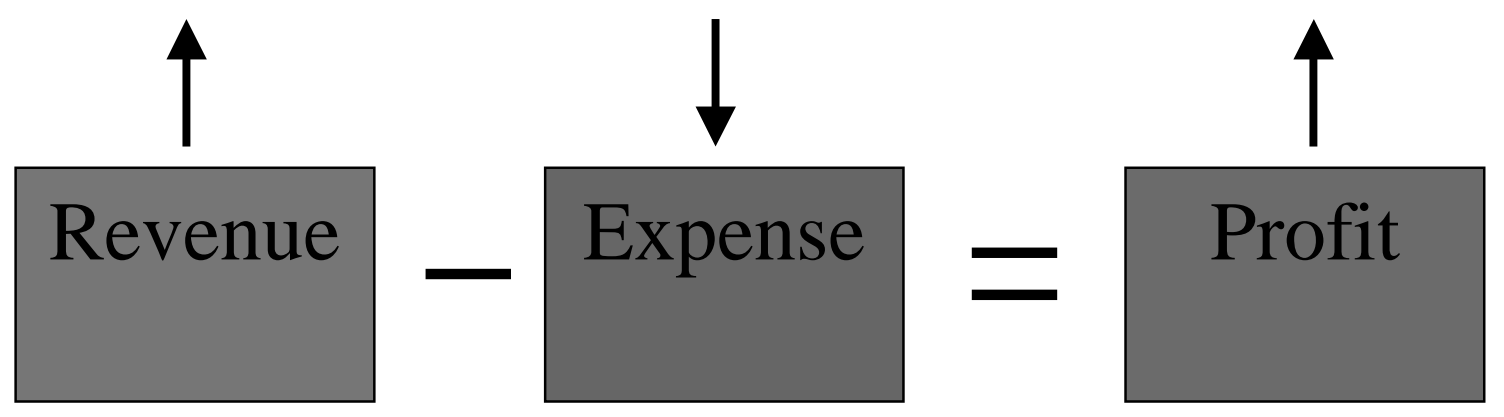

\section{Figure 4-1 Means of Increasing Profit}

Thus, there are effectively two means of profit maximization: reduce expenses and/or increase revenue.

This leads to the approach taken in the analysis in which both of these options are pursued. The most effective means of reducing expenses is to reduce the cost of launch. The first two servicing cases use this approach, by reducing the mass of the spacecraft, thus allowing for the possibility of either putting two spacecraft on one launch vehicle or simply using a smaller, less expensive launch vehicle. 
The other servicing cases take the approach of increasing revenue. This is accomplished by adding more revenue generating transponders to the spacecraft. Although this increases the cost of the spacecraft, the analysis is used to determine if the increased revenue compensates for these increased costs. The baseline and servicing cases are listed below in Table 4-1. The competition cases are listed in Table 4-2. 
Table 4-1 GEO Servicing and Baseline Configuration Cases

\begin{tabular}{|c|c|c|c|c|}
\hline Case & $\begin{array}{l}\text { Servicing } \\
\text { Task }\end{array}$ & $\begin{array}{l}\text { Initial } \\
\text { Orbit }\end{array}$ & Propulsion & $\begin{array}{l}\text { Configuration } \\
\text { Approach }\end{array}$ \\
\hline $\begin{array}{c}\text { Baseline } \\
\text { (Program A) }\end{array}$ & None & $\mathrm{GTO}^{7}$ & Standard Biprop & Current s/c design \\
\hline AQR 1 & $\begin{array}{l}\text { Fuel 1-Time } \\
\text { at Staging } \\
\text { Orbit for OR } \\
\text { and SK }\end{array}$ & Staging $^{9}$ & $\begin{array}{l}\text { Standard Biprop } \\
\text { (launched dry) }\end{array}$ & $\begin{array}{l}\text { Reduced Launch } \\
\text { Cost } \\
\text { 1a: } 2 \mathrm{~s} / \mathrm{c}, 1 \mathrm{LV} \\
\text { 1b: Cheaper LV }\end{array}$ \\
\hline AQR 2 & $\begin{array}{l}\text { Tug for OR } \\
\text { and NSSK }\end{array}$ & Staging & $\begin{array}{l}\text { Biprop used } \\
\text { only for EWSK } \\
\text { and contingency }\end{array}$ & $\begin{array}{l}\text { 2a: Reduced } \\
\text { Launch Cost } \\
\text { 2b: Additional } \\
\text { Transponders }\end{array}$ \\
\hline AQR 3 & Tug for NSSK & GEO & $\begin{array}{l}\text { Biprop used } \\
\text { only for EWSK } \\
\text { and contingency }\end{array}$ & $\begin{array}{c}\text { Additional } \\
\text { Transponders }\end{array}$ \\
\hline AQR 4 & $\begin{array}{l}\text { Refuel before } \\
\text { each NSSK } \\
\text { maneuver }\end{array}$ & GEO & $\begin{array}{c}\text { Refuelable } \\
\text { Biprop Tanks }\end{array}$ & $\begin{array}{c}\text { Additional } \\
\text { Transponders }\end{array}$ \\
\hline AQR 5 & $\begin{array}{l}\text { "Optimal Just } \\
\text { in Time" } \\
\text { Refueling }\end{array}$ & GEO & $\begin{array}{c}\text { Refuelable } \\
\text { Biprop Tanks }\end{array}$ & $\begin{array}{c}\text { Additional } \\
\text { Transponders }\end{array}$ \\
\hline AQR 6 & Tug for OR & Staging & $\begin{array}{l}\text { OR: Biprop } \\
\text { SK: Electric } \\
\text { Propulsion }\end{array}$ & $\begin{array}{l}\text { 6a: Reduced } \\
\text { Launch Cost } \\
\text { 6b: Additional } \\
\text { Transponders }\end{array}$ \\
\hline AQR 7 & Tug for NSSK & Staging & $\begin{array}{c}\text { Use Separable } \\
\text { Biprop Apogee } \\
\text { Stage for OR }\end{array}$ & $\begin{array}{c}\text { Additional } \\
\text { Transponders }\end{array}$ \\
\hline AQR 8 & $\begin{array}{l}\text { Fuel 1-Time } \\
\text { at Staging } \\
\text { Orbit for OR }\end{array}$ & Staging & $\begin{array}{l}\text { OR: Biprop } \\
\text { SK: Electric } \\
\text { Propulsion }\end{array}$ & $\begin{array}{c}\text { Reduced Launch } \\
\text { Cost or } \\
\text { Additional } \\
\text { Transponders }\end{array}$ \\
\hline
\end{tabular}

\footnotetext{
${ }^{7}$ The Geosynchronous Transfer Obit used has perigee at $200 \mathrm{~km}$ altitude (radius $6578 \mathrm{~km}$ ), apogee at $35786 \mathrm{~km}$ altitude (radius $42164 \mathrm{~km}$ or geosynchronous radius) and inclination of approximately 27.5 degrees.

${ }^{8} \mathrm{OR}$ is orbit raising, SK is stationkeeping, and NSSK is North-South Stationkeeping.

${ }^{9}$ The staging orbit is circular at $10600 \mathrm{~km}$ altitude (radius about $17000 \mathrm{~km}$ ) and inclination is 28.5 degrees. It is utilized to minimize fuel consumption of the on orbit servicing architecture.
} 
Table 4-2 GEO Competition Cases

\begin{tabular}{|c|c|c|c|}
\hline Case & Initial Orbit & Propulsion & $\begin{array}{c}\text { Configuration } \\
\text { Approach }\end{array}$ \\
\hline Comp 1 & GEO & $\begin{array}{c}\text { SK: Electric } \\
\text { Propulsion }\end{array}$ & $\begin{array}{c}\text { Additional } \\
\text { Transponders }\end{array}$ \\
\hline Comp 2 & GTO & $\begin{array}{c}\text { OR and SK: Electric } \\
\text { Propulsion }\end{array}$ & $\begin{array}{c}\text { Additional } \\
\text { Transponders }\end{array}$ \\
\hline Comp 3 & GEO & $\begin{array}{c}\text { Use Biprop for } \\
\text { Stationkeeping }\end{array}$ & $\begin{array}{c}\text { Additional } \\
\text { Transponders }\end{array}$ \\
\hline Comp 4 & Staging & $\begin{array}{c}\text { Use Separable } \\
\text { Biprop Apogee } \\
\text { Stage for OR and } \\
\text { Biprop for SK }\end{array}$ & $\begin{array}{c}\text { Additional } \\
\text { Transponders }\end{array}$ \\
& & & \\
\hline
\end{tabular}

Because satellite servicing represents a complete paradigm shift in the commercial satellite market, the assumption in made that it is necessary to completely redesign a spacecraft to optimize spacecraft for servicing. This analysis does not explicitly examine what the new configuration should be; rather it assumes that the same major limitations placed on spacecraft today will still hold in the future. (i.e. mass and volume will continue to be limited) These limitations are primarily dictated by the capacity of launch vehicles and are not expected to change dramatically in the timeline of this potential new market.

\subsubsection{Important Technological Aspects of Each Case}

One important aspect of each case that must be considered is its technological implications, as they relate to the economic viability of the market.

\section{AQR 1}

This case requires a servicing vehicle to dock with and fuel the spacecraft one time on orbit. From a technological standpoint, this case is associated with the least amount of risk because docking and refueling are only required a single time over the lifetime of the spacecraft. In addition, this docking and refueling is performed at a time when there is no risk of interrupting the client spacecraft's services to its clients. 


\section{$A Q R 2$}

This case requires a servicing vehicle to tug the spacecraft from the staging orbit to GEO and then provide stationkeeping tugs approximately every 41 days. From a technological standpoint, using a tug vehicle instead of refueling reduces the risk each time the servicer and client dock because no fluid transfer takes place.

However, using a tug vehicle makes the client spacecraft completely reliant upon the servicing vehicle because the client spacecraft has a very limited supply of contingency fuel on-board, which cannot be refueled. In addition, the frequent dockings (approximately 134 times over the lifetime of the spacecraft) are performed while the client spacecraft is providing service to its own clients. Docking has to be performed in such a way as to maintain the client spacecraft's ability to provide service to its client, which a nontrivial issue.

\section{$A Q R 3$}

This case requires a launch vehicle to insert the client spacecraft directly into GEO and a servicing spacecraft to provide stationkeeping tug maneuvers approximately every 41 days. There is increased risk associated with direct-GEO insertion (DGI), as opposed to launching to a lower orbit: the launch vehicle may under-perform. However, the client spacecraft has a docking mechanism on it, which would allow for a tug vehicle to tug the client to the correct orbit, if the launch vehicle does under perform. As discussed above, from a technological standpoint, using a tug vehicle instead of refueling reduces the risk each time the servicer and client dock because no fluid transfer takes place.

However, several issues presented in $\mathrm{AQR} 2$ apply to $\mathrm{AQR} 3$ as well: client spacecraft is completely reliant upon the servicing vehicle, and docking is performed while the client is providing services.

\section{$A Q R 4$}

This case requires a launch vehicle to insert the client spacecraft directly in GEO and a servicing spacecraft to refuel the client for stationkeeping approximately every 41 days. This case has the same risks, presented in AQR 3, associated with DGI and reliance upon a servicer. However, the client spacecraft is slightly less reliant on the servicer because it 
has contingency fuel that can be replenished if it is used for an emergency. In addition to the risks discussed in $A Q R$ 3, the $A Q R 4$ spacecraft is refueled, which adds the risk of fluid transfer on orbit.

\section{$A Q R 5$}

This case requires a launch vehicle to insert the client spacecraft directly in GEO and a servicing spacecraft to refuel the client for stationkeeping approximately 12 times over the lifetime of the client spacecraft. This case has the same risks, presented in AQR 3, associated with DGI and being reliant upon a servicer. However, the client spacecraft is much less reliant on the servicer because it is refueled much less frequently and has contingency fuel that can be replenished if it is used for an emergency. In addition to the risks discussed in AQR 3, the AQR 5 spacecraft is refueled, which adds the risk of fluid transfer on orbit.

\section{AQR 6}

This case requires a servicing vehicle to tug the spacecraft from the staging orbit to GEO and then the client spacecraft provides its own stationkeeping using electric propulsion. As the servicing provided only requires docking one time on orbit before the client spacecraft begins service to its clients, the risk associated with the servicing is minimal compared to the other cases. Using electric propulsion (EP) for stationkeeping is a less utilized technology than using bipropellant, which may slightly increase its risk. However, EP has been successfully utilized on spacecraft for many years, specifically in Russia. (Wertz and Larson, 1999).

\section{AQR 7}

The idea behind the case is to launch a separable apogee stage to the staging orbit on a less expensive launch vehicle (the Long March), where it docks with the client spacecraft to perform its orbit raising. Once in GEO, the apogee stage separates and the client spacecraft uses a tug vehicle for NSSK. Unfortunately, adding the cost of an additional launch makes the case much more expensive than the other AQR cases, without providing enough additional revenue to compensate for the increased costs. Thus, after the analysis, this case was discarded because it was uneconomical. 


\section{AQR 8}

This case was also discarded after the analysis, because it is severely limited by volume. The case involves launching the spacecraft with an empty orbit raising fuel tank and adding an electric propulsion system onboard. However, the spacecraft does not provide a cheaper launch because it is unable to fit into the smaller launch vehicle fairing of a Delta 2 and is also too large to allow two spacecraft to fit into one Atlas IIAS fairing. It is also limited by volume in terms of adding additional transponders and launching a single spacecraft on an Atlas IIAS. It is limited to the point, where it is not comparable to the other servicing cases.

\section{Comp 1}

This case requires a launch vehicle to insert the client spacecraft directly in GEO and the spacecraft to provide for its own stationkeeping using electric propulsion. As in some of the other cases, there is an increased risk associated with DGI. However, unlike the servicing cases, if the launch vehicle under performs, this spacecraft does not have docking abilities that would allow another spacecraft to perform any necessary orbit raising maneuvers. As discussed above, the use of electric propulsion for stationkeeping adds minimal risk to the spacecraft.

\section{Comp 2}

This case requires a launch vehicle to launch the spacecraft to GTO and then the spacecraft to perform all of its own orbit raising and stationkeeping using electric propulsion. The major issue with using electric propulsion for orbit raising is that it is much slower than using bipropellant. This causes two problems. First, the spacecraft effectively loses a month's worth of revenue (the difference in time between using EP and bipropellant) while it performs its orbit raising maneuvers. This loss of a month of service may cause other issues, as well. For instance, the client could lose market share because it is unable to begin servicing its clients earlier.

In addition to the issues with the revenue stream, using EP for orbit raising exposes the satellite to increased radiation as it passes through the Van Allen belts (Wertz and Larson, 1999). In order to compensate for this the spacecraft must utilize more radiationhardened materials, which increases the cost and mass of the spacecraft. 
After considering the increased time to orbit and the increased radiation exposure, for the purposes of this analysis, the AQR cases will only be compared to the Comp 1 spacecraft. However, the Comp 2 detailed analysis is presented below for completeness.

\section{Comp 3}

Comp 3 uses an Atlas IIAS to launch a spacecraft directly to GEO. The spacecraft then uses its own bipropellant propulsion system to perform all of the necessary stationkeeping maneuvers on orbit. This case is discarded because it is severely mass limited, in terms of the number of additional transponders that can be added to the spacecraft. Using DGI reduces the mass the launch vehicle can handle and the biprop propellant mass needed for stationkeeping also reduces the mass available to additional transponders. The mass limitation translates to revenue limitation, which makes the case only slightly economically better than the baseline case and much worse than the EP competition cases.

\section{Comp 4}

Comp 4 is the same as AQR 7, except that the Comp 4 spacecraft performs its own stationkeeping maneuvers, using its onboard biprop propulsion system. As with the AQR 7 spacecraft, adding the cost of an additional launch makes the case much more expensive than the other cases, without providing enough additional revenue to compensate for the increased costs. Thus, Comp 4 was discarded.

\section{Discarded Cases}

Although each case discussed above underwent the same analysis, the following section does not present details for cases that were discarded due to either economic or technical issues.

\subsubsection{Transforming the Baseline Satellite to the Case Configurations}

To determine the value of each case, each trade begins with a baseline satellite. The baseline satellite represents a commercial GEO satellite designed and built by Space Systems/LORAL (SS/L). The baseline satellite will be referred to as Program A. The quantitative technical and cost information used in this analysis is representative of 
typical spacecraft designs but does not precisely reflect any specific SS/L design in order to protect SS/L proprietary data.

The initial part of each trade is broken into four areas. These areas are mass, volume, launch vehicle and spacecraft cost, and revenue. The mass, volume, and spacecraft costs are based upon Program A data and data from similar programs. The approach is to determine the changes in these areas associated with each trade configuration. Various spacecraft components are subtracted from or added to the baseline satellite in order to "transform" into the appropriate configuration.

After the appropriate parts are subtracted or added to the baseline satellite to form the serviced or competition satellite, the additional volume and mass available for additional transponders is examined. It is necessary to determine if volume or mass is the limiting factor in terms of the number of additional transponders that can be added to the spacecraft. Once the number of additional transponders is calculated, the additional cost and revenue associated with the additional transponders are added to the total cost and total yearly revenue for the satellite. A discounted cash flow analysis is then used to determine the net present value (NPV), before options, of the satellite case. This NPV is the value of the satellite before the flexibility of servicing is taken into account. The value of the option for life extension is then calculated and summed with the NPV to get the total present value of the configuration. The flow chart for this analysis structure is shown below, in Figure 4-2. 


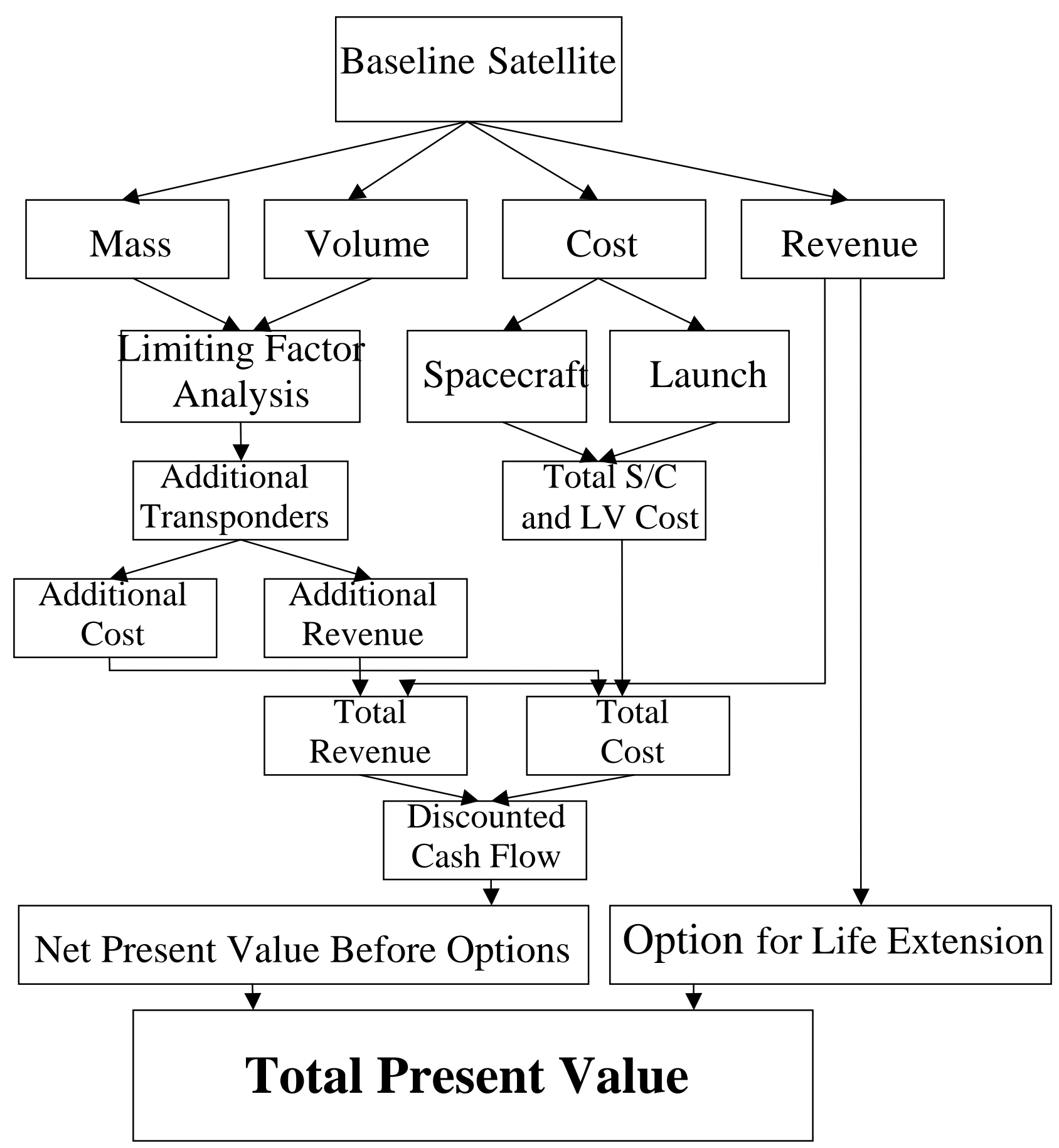

Figure 4-2 Flow-chart for Analysis of Each Case

As there are many different definitions of flexibility, it is important to define the way it is being used in this analysis. For the purpose of this study, flexibility is defined as the ability of a spacecraft to adapt, on-orbit, to changes in its initial requirements and 
objectives. For example, the ability to use servicing to extend the life of a serviced spacecraft is considered flexibility.

Value is another widely used term, which has many different definitions. Value is defined here as economic benefit. In this chapter, it is the economic benefit to the client. The total value, after flexibility is taken into account, is the theoretical upper limit of the amount that the client is willing to pay to have their satellite serviced.

In order to determine the "true" value of servicing, one must take into account the value of flexibility. The two aspects of flexibility discussed in this analysis are the flexibility for life-extension and the flexibility to maneuver a satellite on-orbit to capture a different market. The value of flexibility is added to the value of servicing before flexibility to determine the client's total present value of servicing.

After the value of each case is determined, the servicing cases and competition cases are compared to each other to determine which trade provides the most value to the client.

\subsubsection{Baseline Calculations for Subtraction or Addition of Parts}

The components that are added to or removed from the baseline satellite are different for each case. The components that are removed include fuel tanks, structural mass, pressurant tanks, and propulsion capabilities. The components that are added include fuel tanks, fuel, docking and refueling mechanisms, pressurant tanks, and electric propulsion components. Each of these is discussed below.

\section{Fuel Tanks}

This analysis assumes biprop fuel is used for all maneuvers in the baseline and AQR cases. The Comp cases utilize electric propulsion. The size and mass of the baseline satellite fuel tanks is found using the mass and volume of fuel needed to perform orbit raising maneuvers from a geosynchronous transfer orbit (GTO), North-South stationkeeping (NSSK), and East-West station-keeping (EWSK) maneuvers over the satellite's 15-year on-orbit life.

The rocket equation is the primary equation used to size the fuel tanks: 


$$
\frac{M_{0}}{M}=\operatorname{Exp}\left(\frac{\Delta V}{g I_{s p}}\right),
$$

where the pre-maneuver spacecraft wet-mass is $\mathrm{M}_{0}$, the post-maneuver spacecraft mass is $\mathrm{M}$, the specific impulse, $I_{\mathrm{sp}}=310 \mathrm{~s}$ for orbit raising $(\mathrm{OR})$ and $\mathrm{I}_{\mathrm{sp}}=292 \mathrm{~s}$ for stationkeeping maneuvers, the standard mean acceleration of gravity on earth, $\mathrm{g}=9.8$ $\mathrm{m} / \mathrm{s}^{2}$, and $\Delta \mathrm{v}$ is in $\mathrm{m} / \mathrm{s}$ and varies with the maneuver performed. Since

$$
M_{0}=M+M_{\text {Fuel Used }}
$$

it is straightforward to find the mass of the fuel used during a maneuver. It is simply

$$
M_{\text {Fuel }}=M\left(\operatorname{Exp}\left(\frac{\Delta v}{g I_{s p}}\right)-1\right)
$$

Using the rocket equation with $\Delta \mathrm{v}=1800 \mathrm{~m} / \mathrm{s}$ for orbit raising maneuvers, the following ratio is found:

$$
\frac{M_{G T O}}{M_{B O L}}=1.8
$$

where $\mathrm{M}_{\mathrm{GTO}}$ is the mass of the spacecraft at geosynchronous transfer orbit and $\mathrm{M}_{\mathrm{BOL}}$ is the mass of the spacecraft at the beginning of its life upon reaching geosynchronous orbit (GEO). Given that

$$
M_{\text {GTO }}=M_{\text {Orbit Raising Fuel }}+M_{\text {BOL }}
$$

the mass of the fuel for orbit raising (OR) from geosynchronous transfer orbit is

$$
M_{\text {Fuel OR }}=0.8 M_{B O L} .
$$

In addition to the orbit raising fuel, the baseline satellite must carry fuel for NSSK and EWSK maneuvers. Using the rocket equation with $\Delta \mathrm{v}=750 \mathrm{~m} / \mathrm{s}$, for a 15 year lifetime, and $I_{\mathrm{sp}}=292 \mathrm{~s}$,

$$
M_{B O L}=1.3 M_{E O L}
$$

where $\mathrm{M}_{\mathrm{EOL}}$ is the mass of the spacecraft at the end of its on-orbit life, which is effectively the spacecraft dry mass. Given that 


$$
M_{B O L}=M_{\text {Stationkeeping Fuel }}+M_{E O L}
$$

the mass of the stationkeeping (SK) fuel is

$$
M_{\text {Fuel } S K}=0.3 M_{E O L} \text {. }
$$

Thus, the total fuel onboard is

$$
M_{\text {Fuel Total }}=0.8\left(1.3 M_{E O L}\right)+0.3 M_{E O L}=1.34 M_{E O L}=1.34 M_{D r y} .
$$

Using a baseline dry mass of $1547 \mathrm{~kg}, \mathrm{M}_{\text {Fuel Total }}=2072 \mathrm{~kg}$. The fuel tank mass can now be calculated using

$$
M_{\text {Fuel Tank }}=0.029 M_{\text {Fuel }}
$$

and the volume can be found using ${ }^{10}$

$$
V_{\text {Fuel Tanks }}=\left(\frac{0.0009 \mathrm{~m}^{3}}{\mathrm{~kg} \text { fuel }}\right) M_{\text {Fuel }}
$$

Fuel tank volume and mass are found for the other cases using the same basic method. However, all of the serviced satellites are launched to the staging orbit, which requires more fuel for OR maneuvers to GEO. Using the above method, with $\Delta \mathrm{v}=2395 \mathrm{~m} / \mathrm{s}$, the wet mass at staging is $\mathrm{M}_{\mathrm{STG}}=2.2 \mathrm{M}_{\mathrm{BOL}}$, which leads to

$$
M_{\text {Fuel OR from Staging }}=1.2 M_{B O L} \text {. }
$$

Where the method of fuel tank sizing differs significantly from that presented above, the differences are outlined in the individual case sections.

The cost of the fuel tanks is taken into account as well. The costs of the various fuel tank sizes are from company data. ${ }^{11}$

\footnotetext{
10 The mass of the fuel tank per $\mathrm{kg}$ of fuel is an estimate from a propulsion engineer at Space Systems/LORAL.

${ }^{11}$ The cost data is an estimate from a propulsion engineer at Space Systems/LORAL who is familiar with fuel tank costs.
} 


\section{Pressurant Tanks}

A pressurant tank filled with helium is included in the "dry mass" of the baseline satellite. This gas is included in the dry mass because it will not be refueled on orbit and thus must be taken into account in the mass and volume that is launched into orbit. This mass is referred to as "dry" because it does not include the fuel mass.

The mass and volume of the baseline pressurant tank are calculated because some of the cases require a different size pressurant tank. Thus, in order to calculate the new "dry" mass it is necessary to subtract the standard baseline tank mass and volume and add the mass and volume of the pressurant tank that is appropriate for the individual case.

The Ideal Gas Law is used to calculate the volume of the baseline pressurant tank. Recognizing that the helium must fill the volume of the fuel used for orbit raising at a pressure of $\mathrm{P}_{\text {Fuel Tank }}=180 \mathrm{psi}$ and the helium is stored at $\mathrm{P}_{\text {helium }}=4000 \mathrm{psi},{ }^{12}$ the volume of the baseline pressurant tank is

$$
V_{\text {Baseline He Tank }}=\frac{\left(P_{\text {Fuel Tank }}\right)\left(V_{\text {OR Portion of Fuel Tank }}\right)}{P_{\text {He Tank }}}=0.065 m^{3} .
$$

The calculation of the pressurant tank for each case is shown in the individual case sections.

\section{Propulsion Systems}

As mentioned above, the baseline case is a standard bipropellant propulsion system used for both orbit raising and stationkeeping. Each of the other cases has its own modified propulsion system. For example, in AQR 2, the satellite does not need orbit raising or NSSK capabilities because these maneuvers are performed by a tug. Thus, the parts of the propulsion system that are specific to orbit raising are removed. The redundant parts specific to NSSK are removed as well. The NSSK capability is not removed entirely because that would significantly increase the risk of servicing. The case specific propulsion capability changes are discussed in the individual case sections.

\footnotetext{
${ }^{12}$ The tank pressure, along with information about the functionality of pressurant tanks, is an estimate from a propulsion engineer at Space Systems/LORAL, who is familiar with both the baseline satellite and satellite refueling.
} 
In addition to the changes made to the biprop cases, the electric propulsion cases are discussed in more detail in each of their own sections. As expected, use of electric propulsion requires much larger modifications to the satellite propulsion system.

\section{Docking and Refueling Mechanisms}

In order to service a satellite, the satellite must have a method to interface with the servicing satellite. In the cases where the satellite is tugged, a redundant docking mechanism is needed. In the refueling cases, a redundant docking mechanism and refueling interface are necessary. The mass and cost of each of these items are taken into account in the appropriate cases.

\section{Structural Savings}

The baseline satellite configuration includes a central cylinder and other support structures that serve to support the fuel tanks during the harsh launch environment. When the satellite configuration is changed such that the fuel tanks are either removed or minimized, much of this support structure is unnecessary. Therefore, for each of the cases, the estimated mass and cost of the unnecessary support structure is subtracted from the baseline satellite mass and cost. ${ }^{13}$ The volume of removed support structure is minimal and is neglected in this analysis.

\subsubsection{Mass}

The mass of the satellite is limited only by the capacity of the launch vehicle. The launch vehicle mass capacity for each of the cases is reduced from the baseline case because the servicing cases utilize a different orbit. Four of the servicing cases assume the spacecraft is launched to a staging orbit. This staging orbit is not the same as GTO. The nonstandard staging orbit is necessary because it is an orbit in which minimal propulsion is needed to maintain an on-orbit supply of fuel. Since the servicing cases involving orbit raising require fuel on orbit, whether it is used by the spacecraft itself or a tug vehicle, all of them utilize the same staging orbit. In addition to the satellites being launched to a staging orbit, several of the cases involve direct GEO insertion (DGI), which reduces the

\footnotetext{
${ }^{13}$ The estimates in this analysis are from a satellite structures engineer at Space Systems/LORAL.
} 
launch vehicle capacity even further. The total mass capacity for launch is $85 \%$ and $76 \%$ of the mass capacity to GTO, for spacecraft launched directly to staging and GEO, respectively (Mission Planner's Guide for Atlas Launch Vehicle Family, Rev. 5, 1995) and (Turner, Ref. 2, 2000).

The final result of the mass analysis is the extra mass available for payload. Ultimately, this number along with the extra available volume determines the number of additional transponders that can be added to the satellite. Taking the dry mass of Program A and subtracting or adding components as necessary determines the extra mass. For example, if a satellite is going to be continually refueled, the standard fuel tanks, pressurant tank, and some of the structural mass can be removed from the satellite. In their place, smaller fuel tanks, refueling and docking mechanisms and a smaller pressurant tank must be included in the new configuration. This subtraction and addition, along with the constraint of launch vehicle capacity leads to a $\Delta$ mass, which can be filled with revenue generating transponders.

\subsubsection{Volume}

The volume calculations are only slightly more complicated than the mass calculations. The same basic approach of subtraction or addition of parts is taken. The difference lies in that the volume of the baseline satellite is assumed to fill the launch vehicle fairing, thus the launch vehicle volume capacity is not examined for each case individually. It is however, crucial to the case $\mathrm{AQR} 1$, as this case tries to reduce the cost of launching the satellite by either putting two spacecraft on one launch vehicle or one spacecraft on a smaller, less expensive launch vehicle. This requires redesign of the spacecraft, such that it can utilize the entire volume of the fairing, including the upper cone-shaped section.

\subsubsection{Spacecraft Cost}

Spacecraft cost is taken into account by finding a $\Delta$ cost for each case. This is accomplished by tracking the changes associated with each case and estimating their corresponding increase or decrease in cost. For each case, this $\Delta$ cost and a $\Delta$ (transponder 
cost) are then added to an assumed baseline cost of $\$ 95 \mathrm{M}$ to find the total cost of the new satellite. ${ }^{14}$

Another important aspect to spacecraft cost is the cost of redesign. As each of the cases would require some level of redesign, this cost could have a significant impact on the overall cost of the satellite. The cost of redesign is assumed to be $\$ 40 \mathrm{M}$ or $\$ 60 \mathrm{M}$, for the cases where the number of transponders are decreased or increased from the baseline case, respectively. ${ }^{15}$ The expected redesign cost for each case is amortized over the expected number of satellites that will utilize that particular servicing configuration. It is assumed that a maximum of one servicing configuration will be adopted, as the infrastructures behind the various servicing options are very different, expensive, and complex.

\subsubsection{Launch Vehicle Cost}

For many commercial GEO satellites, launch vehicle costs are approximately equivalent the cost of the spacecraft itself. The baseline launch vehicle in this study is the Atlas IIAS. Although it is recognized that this launch vehicle will probably not be available during the timeline that satellite servicing will be commercially available, the basic launch technology and relative costs are believed to be remaining fairly constant throughout this time period. Thus, even if another launch vehicle is used in the future, the relative costs are assumed to scale with the mass and volume capacity of the launch vehicle, which allows a current launch vehicle to be used in this analysis.

The baseline launch cost is for an Atlas IIAS carrying one spacecraft to GTO. This cost is $\$ 97.5 \mathrm{M}$ (Isakowitz, 1999). This is simply an average of the low and high estimates for the Atlas IIAS launch vehicle cost. The Atlas IIAS was chosen as the baseline launch vehicle for several reasons. First, its mass limit to GTO is very close to the baseline satellite mass. This reduces the impact of excess launch vehicle mass capability on the

\footnotetext{
${ }^{14}$ The Program A cost is from a business development employee at Space Systems/LORAL who is familiar with the actual cost of Program A. This number is not the actual cost of the spacecraft, as that number is proprietary, but is within $\$ 5 \mathrm{M}$.

15 The redesign costs are estimates from a research and development employee as Space Systems/LORAL. The employee is has extensive experience with redesigning satellites and the costs associated with redesign.
} 
number of additional transponders. Second, it has a very high reliability (100\% to date). This minimizes the effect of launch risk on the analysis.

As the staging orbit is not a standard orbit for current launch vehicles, it is assumed that the cost of the launch would be increased by $10 \%$. This increase in cost is assumed to account primarily for increased costs in software. In reality these costs would be a onetime expense to the launch vehicle manufacturer, who would pass these costs on to the client requesting an unconventional launch. However, to remain conservative, a 10\% launch cost increase is included for all satellites launched to the staging orbit.

No additional increase in cost is assumed for the cases that use one launch vehicle for two spacecraft. This is because the conservative approach of including a $10 \%$ launch cost increase and using an average baseline cost, is assumed to properly account for any additional expenses associated with adding an additional spacecraft to the launch vehicle.

\subsubsection{Limiting Factor Analysis}

The limiting factor analysis is used to determine the number of additional transponders that can be added to the satellite in each case. It begins with the additional volume and mass generated by changing the satellite configuration. The additional number of transponders that can be added is calculated using the following:

$\underset{\text { Transponders }}{\text { Additional }}=\min \left(\frac{\text { Additional Volume }}{\text { Incremental Transponder Volume }}, \frac{\text { Additional Mass }}{\text { Incremental Transponder Mass }}\right)$

The incremental transponder volume is the additional volume associated with adding one more transponder to the spacecraft. This volume is the volume of the transponder itself, as well as the volume for access, packaging, scaleable batteries, waveguide, and OMUX (output multiplexer). ${ }^{16}$ Adding the volumes above, the incremental transponder volume is $0.0227 \mathrm{~m}^{3}$.

The incremental transponder mass is the mass of all of the parts of the baseline satellite that scale with the number of transponders divided by 48 , the total number of

\footnotetext{
${ }^{16}$ The volume of each of these parts is from Space Systems/LORAL internal documents.
} 
transponders on the satellite. ${ }^{17}$ The incremental transponder mass used in this analysis is $27.45 \mathrm{~kg}$.

\subsubsection{Revenue and Cost Calculations}

The calculations for the final revenue/year and cost of the spacecraft begin with the baseline satellite revenue/year and cost. As revenue and cost numbers are generally proprietary, the numbers used are not exact numbers from Program A.

\section{Cost}

The cost of the baseline spacecraft is assumed to be $\$ 95 \mathrm{M}$. The launch cost for the baseline satellite is $\$ 97.5 \mathrm{M}$. This is the current cost of an Atlas IIAS launched to GTO (Isakowitz, 1999). This leads to a total baseline spacecraft and launch cost of $\$ 192.5 \mathrm{M}$.

In addition to the baseline cost, each case has an associated $\Delta$ (spacecraft cost) (the change in the cost due to adding and subtracting components and the cost of redesign), a $\Delta$ (launch cost) (the change in the launch cost due to using a non-standard orbit and/or non-standard configuration), and the cost of adding additional transponders. These costs are summed to determine the total spacecraft and launch cost for each case.

The $\Delta$ (spacecraft cost) is calculated by adding or subtracting the cost of components as they are added or subtracted from the baseline satellite and accounting for the cost of redesigning the spacecraft. The costs of individual components, for example a fuel tank, are generally very small in comparison to the other cost changes. However, they are included in the analysis for the sake of completeness.

\section{Revenue}

The baseline revenue/year is calculated as follows:

$$
\begin{aligned}
& \frac{\text { Revenue }}{y r}=\left(\frac{\text { Revenue }}{\text { Transponder } \times y r}\right)(\# \text { transponders on baseline spacecraft }) \\
& =\$ 2.16 M \times 48 \text { transponders }=\$ 103.8 M
\end{aligned}
$$

\footnotetext{
${ }^{17}$ The incremental mass is calculated using Space Systems/LORAL data from Program A.
} 
The revenue/transponder/year is from The London Satellite Exchange, an online market place for the satellite industry, which sells capacity on spacecraft ("The London Satellite Exchange", 2001). As each market values bandwidth differently (e.g. bandwidth over the U.S. is generally more economically valuable than bandwidth over Africa) an average over the globe is used. In addition, the satellite exchange offers both $\mathrm{C}$ Band and $\mathrm{Ku}$ Band capacity. These numbers are averaged, as well.

It is recognized that the servicing timeline is long and it is very probable that other bands will be used by the time servicing is utilized. For the purpose of this analysis, it is assumed that the revenues will scale with costs in the future.

The total revenue/year for each case is calculated as follows:

$$
\frac{\text { Total } \operatorname{Revenue}}{Y r}=(48 \text { transponders }+ \text { additional transponders })\left(\frac{\$ 2.16 M}{\text { transponder }}\right) \text {. }
$$

\subsubsection{Discounted Cash Flow Analysis}

After revenue and cost numbers are generated for each case the net present value (NPV) for the case is calculated using a discounted cash flow (DCF). The DCF analysis is a realistic representation of the way current satellites are financed. ${ }^{18}$ The model includes taxes, depreciation, debt and equity financing, interest expenses, and satellite operating costs. The model is set up such that the inputs are spacecraft revenue per year and total cost of a spacecraft (including launch). The model calculates the NPV based on a given discount rate. For the purposes of this study the following assumptions are made in the analysis:

- Corporate tax rate $=40 \%$

- $\quad$ Loan amount $=85 \%$ of cash needed

- Depreciation life $=15$ years

- Cost of Debt $=10 \%$

\footnotetext{
18 The DCF model is a modified version of a model used by Space Systems/LORAL employees to determine the viability of new projects.
} 
- Spacecraft and launch costs are distributed evenly over two years before launch date

- Operating Expenses $=15 \%$ of gross revenue generated per year after launch

- Operating Expenses $=\$ 3 \mathrm{M} / \mathrm{year}$ for two years before launch

- Discount rate $=10 \%$ (this number is varied to show sensitivity to cost of capital)

For the baseline case the NPV is calculated using the above assumptions, a total cost of $\$ 192.5 \mathrm{M}$, and revenue per year of $\$ 103.8 \mathrm{M}$. The baseline NPV is $\$ 179.3 \mathrm{M}$.

\subsubsection{Option to Extend Life of Satellite}

An additional benefit to on-orbit servicing is that it provides the possibility of extending the life of the satellite beyond its design lifetime. Assuming the satellite is still operational at the end of its lifetime and the market that it serves is still willing to pay for its services, a tug or refueling spacecraft can be used to maintain the satellite's orbit to allow it to continue to generate revenues.

It is recognized that some spacecraft would be unable to take advantage of this life extension provided by servicing because either the spacecraft has already reached the end of its useful life or the market it serves is not viable. These are taken into account in the following analysis by multiplying the value given by the equation below by the percentage of spacecraft that would be operational and have a market available to them after their 15-year lifetime.

The approach to determining the value of the option for life extension is to apply a modified version of the Black-Scholes equation. (See Section 2.3.6) As it applies to life extension, the Black-Scholes equation is

$V_{\text {OPTION }}=S_{0} \times N\left(d_{1}\right)-e^{-r T_{0}} \times\left(E+C_{\text {ops }}\right) \times N\left(d_{2}\right)$ 
where

$$
\left\{\begin{array}{l}
N(x)=\int \frac{1}{\sqrt{2 \pi}} e^{-t^{2}} d t \\
d_{1}=\frac{\left[\ln \left(S_{0} / E\right)+\left(r+\sigma^{2} / 2\right) \times T_{0}\right]}{\sigma \sqrt{T_{0}}} \quad N \text { : cumulative normal distribution function } \\
d_{2}=d_{1}-\sigma \sqrt{T_{0}}
\end{array}\right.
$$

and

$\mathrm{S}_{0} \quad=$ present value of revenue stream over life extension

$\mathrm{r} \quad=$ risk-free interest rate

$\mathrm{E} \quad=$ cost of servicing

$\mathrm{C}_{\mathrm{ops}}=$ operating cost of spacecraft over life extension

$\mathrm{T}_{0} \quad=$ time of servicing for life extension (i.e. design lifetime of satellite)

$\sigma \quad=$ volatility of the revenues per year of (continuously compounded) rate of return

(Saleh, 2001)

Although each of the variables above is important in the options analysis, the value of the option is more sensitive to some of the variables than others. This is important because the sensitivity of the final answer to each variable should determine the effort put into finding accurate inputs for each of the variables. Thus, a sensitivity analysis was performed on the option value to determine which inputs need to be very accurate. In the range of reasonable inputs and values for this analysis, it was found that the value of the option for life extension was sensitive to the length of life extension, which determines $\mathrm{S}_{0}$; the risk-free interest rate; and the percentage of satellites that, if not limited by fuel, are operational after their 15-year lifetime.

The value of the revenue stream over the life extension, $S_{0}$, is simply the revenue generated per year by the satellite in question, multiplied by the length of life extension. 
The risk-free interest rate is 5.39\% ("Federal Reserve Statistical Release", 2002). The cost of servicing, E, is assumed to be the cost of delivery of the fuel to GEO using Aquarius multiplied by the amount of fuel necessary to maintain the satellite's orbit over the length of time of life extension. The cost of the servicing infrastructure is not included, as it is accounted for in the cost of servicing over the design lifetime of the satellite. The operating cost of the spacecraft over the life extension is assumed to be $10 \%$ of revenues per year ${ }^{19}$. The time of servicing, $\mathrm{T}_{0}$, is assumed to be 15 years. The volatility of the revenues per year is assumed to be 0.087 (Christodoulou, 2001). In addition, the average life extension is assumed to be 2.5 years $^{20}$.

In the appropriate cases (i.e. the cases that use either a tug or refueling) the equation above is used to determine the option value. As mentioned above, not all satellites will be operational after their 15-year design lifetime, which means that not all spacecraft will be able to take advantage of life extension. As this must be reflected in the option value, the value calculated above is multiplied by $70 \%$, the percentage of satellites that are expected to be operational after their design lifetime. ${ }^{21}$ The option value is then added to the NPV for each case to determine the total value of servicing.

\subsubsection{Flexibility to relocate satellite and capture other markets}

Another advantage to servicing is that it gives the client the ability to maneuver its spacecraft to cover other markets without shortening the useful lifetime of the spacecraft. This flexibility has economic value because it allows the client spacecraft to adapt to changing market conditions. For example, market conditions today may indicate that there is an economically viable market in Africa but a few years after beginning service the market collapses, which significantly reduces the revenue stream for the owner of the spacecraft. However, there may be a market for the same services in Asia. With the help of a tug or refueling vehicle, the serviced satellite can relocate over the new market and

\footnotetext{
${ }^{19}$ This number is an estimate from a finance employee at Space Systems/LORAL, who performs financial valuations.

${ }^{20}$ This number is an estimate from a systems engineering employee at Space Systems/LORAL.

${ }^{21}$ This number is an estimate from two independent sources at Space Systems/LORAL.
} 
continue to generate revenue without shortening its lifetime because it uses its limited fuel supply to perform a fuel-intensive orbit maneuver.

Although this flexibility has value and should be considered within the context of the value of satellite servicing, assigning a dollar value to this flexibility for a general case is not a trivial issue. First, it is necessary to know which are the initial and possible final markets. This information is needed to determine the amount of fuel needed to maneuver the spacecraft from the initial to final market. In addition, the volatility of each of these markets must be known as well as the probability that the possible final market will be served by another satellite before the spacecraft in question utilizes the option to relocate there. As these numbers are associated with a particular satellite and not the general case, this analysis should be performed by or for a specific client, in order to determine the value of the option for relocation.

\subsection{AQR 1: Current Satellite Launched Dry}

\subsubsection{Description}

This case examines the client's perspective on the economic viability of launching a slightly modified version of the baseline spacecraft, without fuel, to the staging orbit. The spacecraft is fueled once at the staging orbit, with enough fuel to perform its OR maneuvers and a lifetime of NSSK and EWSK maneuvers. The approach to increasing profit for AQR 1 is to reduce launch costs. This is accomplished because the mass of the dry spacecraft is such that two can be launched on the baseline launch vehicle or one can be placed on a smaller, less expensive vehicle. Thus, AQR 1 is broken down into AQR 1a and AQR 1b, two spacecraft on one launch vehicle and one spacecraft on a smaller launch vehicle, respectively. As the calculations for both $1 \mathrm{a}$ and $1 \mathrm{~b}$ are very similar, they will be presented together throughout this section.

\subsubsection{Mass}

The mass of the serviced spacecraft is found by subtracting the mass of unnecessary components and adding the mass of required components to the baseline spacecraft mass. 


\section{Fuel Tanks}

Although the serviced satellites in this case are essentially the baseline satellite launched dry, it is necessary to re-size the fuel tanks in order to compensate for the increased fuel needed to perform OR from the staging orbit. The mass of the fuel needed for OR and stationkeeping is

$$
M_{\text {Fuel }}=1.86 M_{\text {Dry }}
$$

where $\mathrm{M}_{\text {Dry }}$ is the dry mass of the spacecraft. This dry mass is dictated by the capacity of the launch vehicle. Since AQR 1a and $1 \mathrm{~b}$ use different launch vehicles, their dry masses are different. In AQR 1a, the launched dry mass of the satellite is half of the capacity of the Atlas IIAS, after adapters are considered, which is $1480.6 \mathrm{~kg}$. This leads to a fuel mass of $2754 \mathrm{~kg}$ and a fuel tank mass of

$$
M_{\text {Fuel Tank }}=0.029 M_{\text {Fuel }}=80 \mathrm{~kg} .
$$

The fuel tank mass for AQR $1 \mathrm{~b}$ is found using the same method. The dry mass of the satellite is the launch capacity of the Delta 2/7295 to the staging orbit, which is 1588.7 $\mathrm{kg}$. This leads to a fuel tank mass of $86 \mathrm{~kg}$.

\section{Pressurant Tanks}

As with the fuel tanks, it is necessary to re-size the helium pressurant tanks for AQR 1a and $1 \mathrm{~b}$ to account for the increased fuel needed for OR from the staging orbit. As with the other components, the approach here is to subtract the baseline satellite pressurant tank, which has a mass of $23 \mathrm{~kg}$, and add the newly sized pressurant tanks.

The mass of a pressurant tank is proportional to its volume. Thus, the volume of the pressurant tank is calculated and the mass is found by linearly scaling it with the mass of the baseline pressurant tank. Since the helium is used only to pressurize the volume of the fuel tank left empty after the orbit raising maneuvers are complete, the volume of the helium tank can be found by using the Ideal Gas Law. Therefore volume of the helium tank is

$$
V_{\text {He Tank }}=\frac{\left(P_{\text {Fuel Tank }}\right)\left(V_{\text {OR Fuel }}\right)}{P_{\text {He Tank }}} .
$$


The mass of the tank is found using

$$
M_{\text {He Tank }}=M_{\text {Baseline He Tank }}\left(\frac{V_{\text {He Tank }}}{V_{\text {Baseline He Tank }}}\right) .
$$

This leads to pressurant tank masses of 33 and $35 \mathrm{~kg}$ for AQR 1a and 1b, respectively.

\section{Propulsion System}

As both the AQR 1a and 1b spacecraft use the same propulsion system as the baseline satellite, it is not necessary to add or subtract components. The mass of the propulsion system remains the same.

\section{Docking and Refueling Mechanisms}

The AQR 1 satellites requires the capability to dock with and be refueled by a servicing satellite. Thus, they need both a docking mechanism and a refueling mechanism. The redundant docking mechanism has a mass of $32 \mathrm{~kg}$ and the refueling mechanism has a mass of $50 \mathrm{~kg}$. This leads to a mass addition of $82 \mathrm{~kg}$ to the baseline satellite. ${ }^{22}$

\section{Structural Savings}

A mass savings is realized for the AQR 1 spacecraft because the central cylinder and other associated structural parts do not need to support full fuel tanks during launch. Thus, a savings of $20 \mathrm{~kg}$ is realized for both AQR $1 \mathrm{a}$ and $1 \mathrm{~b}$ satellites. $^{23}$

\section{Launch Vehicle Capacity}

As both of the AQR 1 spacecraft are launched directly to the staging orbit, there is an associated mass penalty. The launch vehicle mass capacity to staging is $85 \%$ of the capability to the baseline GTO. The total mass capacity for Atlas IIAS to GTO is 3719 $\mathrm{kg}$ and the mass capacity for Delta 2/7295 to GTO is $1869 \mathrm{~kg}$ (Mission Planner's Guide for the Atlas Launch Vehicle Family, 1992) and (Delta II Payload Planners Guide, 2000). In addition, using one launch vehicle for two spacecraft requires an additional launch

\footnotetext{
22 These masses are from Orbital Express documents, internal to Space Systems/LORAL.

23 All structural mass and cost savings estimates are from a structural engineer from Space Systems/LORAL, who is familiar with structural cost and mass savings associated with configuration changes.
} 
vehicle adapter. The mass of each adapter is assumed to be $100 \mathrm{~kg}$. This leads to a single-spacecraft mass capacity of $1481 \mathrm{~kg}$ and $1589 \mathrm{~kg}$ for AQR 1a and 1b respectively.

\section{Result}

After the components discussed above and the launch vehicle capacity are taken into account, a final $\Delta$ mass is found for both AQR $1 \mathrm{a}$ and $1 \mathrm{~b}$. The calculations for each case are shown in Appendix A.

The $\Delta$ mass for AQR $1 \mathrm{a}$ is $-157.8 \mathrm{~kg}$. The negative sign indicates that the final $\mathrm{AQR} 1 \mathrm{a}$ spacecraft mass, before the addition or subtraction of transponders, is greater than the launch vehicle capacity. This occurs because AQR 1a requires two spacecraft on one launch vehicle. The $\Delta$ mass for $\mathrm{AQR} 1 \mathrm{~b}$ is $-58.0 \mathrm{~kg}$. Again, the negative sign indicates that the redesigned AQR $1 \mathrm{~b}$ spacecraft is greater than the launch vehicle capacity. The effect of having negative $\Delta$ masses is discussed in the limiting factor analysis.

\subsubsection{Volume}

The primary volume issue for the AQR 1 spacecraft is whether or not two redesigned spacecraft can fit into one Atlas IIAS and/or whether one re-designed spacecraft can fit into the fairing of a Delta 2/7925. In order to determine this, it is necessary to calculate the change in volume over the baseline satellite. The primary drivers of this change in volume are the fuel tank and the pressurant tank. These are discussed below.

After determining the change in volume from the baseline case, the new total volume is calculated and compared to the size of the two respective launch vehicle fairings. It is then determined if either or both cases are feasible.

\section{Fuel Tanks}

As more fuel is needed to perform the OR maneuvers from the staging orbit to GEO than in the baseline case, the volume of the fuel tanks increases. This new volume is found by scaling it with the fuel tank mass found in Section 4.4.2. The method for this is outlined in the fuel discussion of Section 4.3.3. The baseline fuel tank volume is $1.9 \mathrm{~m}^{3}$. The resized fuel tank volumes are 2.5 and $2.7 \mathrm{~m}^{3}$ for AQR $1 \mathrm{a}$ and $1 \mathrm{~b}$, respectively. 


\section{Pressurant Tanks}

As the volume of the fuel necessary to maneuver to GEO from the staging orbit is greater than the volume of fuel needed to maneuver to GEO from the baseline GTO orbit, the volume of the helium needed to fill the fuel volume is greater than in the baseline case. The equation for the volume of the pressurant tank is presented in the pressurant tanks discussion in Section 4.4.2. The deleted baseline pressurant tank has a volume of 0.065 $\mathrm{m}^{3}$, while the volume of the AQR 1a pressurant tank is $0.094 \mathrm{~m}^{3}$ and $1 \mathrm{~b}$ pressurant tank is $0.100 \mathrm{~m}^{3}$.

\section{Launch Vehicle Capacity}

The launch vehicle volume capacity is a concern for the AQR 1a spacecraft because two spacecraft are being launched on one launch vehicle. Twice the AQR 1a spacecraft volume is compared to the volume capacity of the Atlas IIAS fairing to determine that it is possible to fit two of the redesigned spacecraft into one fairing.

The AQR $1 \mathrm{~b}$ volume is also an issue, from the perspective of launch vehicle capacity, because it must fit into the volume of the Delta 2/7925 fairing. The AQR $1 \mathrm{~b}$ volume is calculated and compared to the volume of the Delta 2/7925 fairing to determine if it is possible to fit one redesigned spacecraft into the smaller fairing.

\section{Result}

After re-sizing the baseline fuel tanks and helium pressurant tanks, the differences between the volume of the baseline case and the serviced spacecraft are 0.6 and $0.8 \mathrm{~m}^{3}$ for $\mathrm{AQR} 1 \mathrm{a}$ and $1 \mathrm{~b}$, respectively.

After subtracting this volume from the volume of the baseline spacecraft, each case volume is compared to the volume of its respective launch vehicle fairing. In $A Q R$ 1a the Atlas IIAS will support two-redesigned spacecraftlb (Mission Planner's Guide for the Atlas Launch Vehicle Family, 1992). In AQR, however, the spacecraft volume is larger than the volume of the Delta 2/7925 fairing by $-1.8 \mathrm{~m}^{3}$ (Delta II Payload Planners Guide, 2000). Thus, the AQR $1 \mathrm{~b}$ spacecraft is not a viable option, because reducing its size by $1.8 \mathrm{~m}^{3}$ completely depletes its revenue generating capabilities. As the AQR $1 \mathrm{~b}$ satellite is not a feasible option, it will not be discussed further in this section. 


\subsubsection{Spacecraft Cost}

The $\Delta$ (spacecraft cost), neglecting the cost of redesign and the cost of a change in the number of transponders, is the cost of the refueling interface and docking mechanism minus the value of structural savings. For AQR $1 \mathrm{a}$, this leads to a $\Delta$ (spacecraft cost) of $\$ 4.46 \mathrm{M}$.

The cost of redesign for AQR $1 \mathrm{a}$ is assumed to be $\$ 40 \mathrm{M}$ to account for redesigning the satellite such that it can take advantage of the upper section of the launch vehicle fairing. This cost is amortized over 12 spacecraft, thus leading to a per spacecraft redesign cost of $\$ 3.33$ M. Summing this with the other changes in spacecraft cost gives a total $\Delta$ (spacecraft cost) of $\$ 7.8 \mathrm{M}$.

\subsubsection{Launch Vehicle Cost}

In AQR 1a, because there are two spacecraft on one launch vehicle, half of the cost of using an Atlas IIAS to launch to the staging orbit is subtracted from the baseline launch cost to get a $\Delta$ (launch cost) per spacecraft of $-\$ 43.9 \mathrm{M}$. The negative sign indicates the spacecraft owner saves $\$ 43.9 \mathrm{M}$ on launch costs for each spacecraft.

\subsubsection{Limiting Factor Analysis}

The limiting factor analysis is used for each case to determine the number of transponders that must be subtracted or can be added to the baseline spacecraft, dependent upon the constraints of the launch vehicle. The two constraints that are analyzed are mass and volume.

After calculating the volume of the AQR 1a spacecraft and the useable fairing volume for the launch vehicle to confirm that two AQR 1a spacecraft can fit onto an Atlas IIAS, it is found that mass is the limiting factor in determining the number of additional or fewer transponders. In order to fit two spacecraft on an Atlas IIAS, it is necessary to reduce each spacecraft's mass by $158 \mathrm{~kg}$. This corresponds to reducing the number of transponders by 6 . 


\subsubsection{Revenue and Cost}

\section{Cost}

The total $\Delta$ cost of the spacecraft and launch vehicle is calculated for each case by adding the $\Delta$ costs associated with the spacecraft, additional transponders, and the launch vehicle. In $A Q R$ 1a, the change in the cost associated with reducing the number of transponders by six is $-\$ 10.1 \mathrm{M}$. Adding this change to the other cost changes gives a total cost decrease of $-\$ 46.2 \mathrm{M}$ over the cost of the baseline satellite and launch. This leads to a total spacecraft and launch cost of $\$ 146.3 \mathrm{M}$.

\section{Revenue}

The total revenue/year is the baseline revenue/year plus the change in revenue/year. The change in revenue/year is proportional to the change in the number of transponders. In $\mathrm{AQR} 1 \mathrm{a}$, the decrease in revenue/year is $\$ 13.0 \mathrm{M}$. This leads to a total revenue per year of $\$ 90.8 \mathrm{M}$.

\subsubsection{Discounted Cash Flow and Options}

Using the revenue and cost numbers discussed above, the NPV for AQR 1a is calculated using a DCF analysis. An NPV of $\$ 169.4 \mathrm{M}$ is found using revenues of $\$ 90.8 \mathrm{M} / \mathrm{yr}$, total costs of $\$ 146.3 \mathrm{M}$, and a discount rate of $10 \%$.

In addition to the NPV, the value of the option for life extension is calculated using the method outlined in Section 4.3.11. The option value is found to be $\$ 59.9 \mathrm{M}$. The NPV and life extension option value are summed to get the total present value of $\$ 229.3 \mathrm{M}$.

\subsection{AQR 2: Launch to Staging Orbit and Tug for Orbit Raising (OR) and North-South Stationkeeping (NSSK)}

\subsubsection{Description}

This case examines the client's perspective on the economic viability of launching a redesigned spacecraft to the staging orbit and then using a tug vehicle to perform its OR 
and NSSK maneuvers. By using a tug vehicle for OR and NSSK, a large part of the fuel mass and volume can be removed. As mentioned previously, there are two means of increasing profit when there is excess volume and mass available on a spacecraft. Thus, this case is broken down into AQR $2 \mathrm{a}$ and AQR 2b. AQR 2a examines the effect of reducing launch cost by putting two spacecraft on one launch vehicle. AQR $2 \mathrm{~b}$ examines the effect of filling the excess mass and volume capacity with additional transponders. As the calculations for each case are very similar, they will be presented together throughout this section.

\subsubsection{Mass}

As in the other cases, the serviced spacecraft is formed by subtracting the mass of unnecessary components and adding the mass of required components to the baseline spacecraft mass.

\section{Fuel Tanks}

This case requires substantial changes in the fuel tank mass and volume. As the satellite does not have to perform its own OR or NSSK maneuvers, the fuel tanks associated with these functions are removed. However, the satellite must maintain its ability to do EWSK and have a small amount of contingency fuel onboard for NSSK.

The approach to determining the $\triangle$ mass between the baseline and AQR 2 satellites is to subtract the entire fuel tank, which accounts for OR, NSSK, and EWSK fuel storage, from the baseline satellite and then to add the fuel tank and fuel associated with EWSK and NSSK contingency. The baseline fuel tank is sized using the method outlined in the fuel tanks discussion in Section 4.3.3.

The reason just the tank volume for OR and NSSK is not simply subtracted, allowing for just the EWSK storage capacity to be left, is because the final spacecraft masses are different in AQR 2a and AQR 2b. This leads to different amounts of necessary EWSK and NSSK contingency fuel for the life of the spacecraft.

In order to determine the mass of the fuel tanks for EWSK and NSSK contingency, the fuel needed for these maneuvers is calculated. Although the fuel needed for each case is different, the same method is used. First, the EWSK fuel is calculated. After 
manipulation of the rocket equation, the mass of the EWSK fuel/kg of spacecraft is found to be

$$
\frac{\mathrm{M}_{\text {EWSK fuel }}}{M_{0}}=1-e^{-\frac{\Delta V}{g I_{s p}}}=\frac{0.013 \mathrm{~kg} \text { fuel }}{\mathrm{kg} \text { spacecraft }}
$$

where $\Delta \mathrm{V}=(2.0 \mathrm{~m} / \mathrm{s} / \text { year })^{*}($ Lifetime of Spacecraft in Years $)$ and $\mathrm{I}_{\mathrm{sp}}=230 \mathrm{sec}$. This ratio is then multiplied by the mass of the spacecraft to find the total mass of EWSK fuel needed over the lifetime of the satellite. The NSSK fuel is calculated using the same method, except that it is calculated for only 1 maneuver. This allows the number of NSSK maneuvers that can be supported by the contingency fuel to be varied more easily. For the purposes of this analysis, the NSSK contingency fuel is assumed to support two NSSK maneuvers. This leads to a contingency fuel per $\mathrm{kg}$ of spacecraft of

$$
\frac{\mathrm{M}_{\text {NSSK Contingency fuel }}}{M_{0}}=\frac{0.004 \mathrm{~kg} \text { fuel }}{\mathrm{kg} \text { spacecraft }}
$$

where a $\Delta \mathrm{V}$ of $5.6 \mathrm{~m} / \mathrm{s} / \mathrm{NSSK}$ maneuver and an $\mathrm{I}_{\mathrm{sp}}$ of $268 \mathrm{sec}$ are used.

After using these equations to find the mass of the EWSK and NSSK contingency fuel, the mass of the fuel tanks can be found using the linear relationship found in the fuel tanks discussion in Section 4.3.3. The masses of the fuel and fuel tank are then added to the baseline satellite mass.

\section{Pressurant Tanks}

As the spacecraft in AQR 2 only performs EWSK maneuvers on its own, it does not need a helium pressurant tank. Thus, the pressurant tank associated with Program A is deleted from the baseline satellite in the process of forming the AQR 2 spacecraft. This leads to a mass savings of $23 \mathrm{~kg}$ for both $\mathrm{AQR} 2 \mathrm{a}$ and $\mathrm{AQR} 2 \mathrm{~b}$.

\section{Propulsion System}

Since the AQR 2 spacecraft does not perform its own orbit raising maneuvers, it does not need a main satellite thruster. It also only needs NSSK capabilities for contingency operations. Thus, the main satellite thruster and one level of redundancy for NSSK thrusters are deleted from the baseline satellite. It is not necessary to add any additional 
propulsion capabilities to the baseline satellite to form the AQR 2 spacecraft. This leads to a mass savings of $20 \mathrm{~kg}$ for both AQR $2 \mathrm{a}$ and AQR $2 \mathrm{~b}^{24}$

\section{Docking and Refueling Mechanisms}

As the AQR 2 spacecraft utilizes a tug vehicle to perform its OR and NSSK maneuvers, it is necessary to add docking capabilities to the baseline satellite. Two sets of identical docking mechanisms are added to the baseline satellite, to provide for adequate redundancy. Each of the tug mechanisms has a mass of $16 \mathrm{~kg}$ (Orbital Express Presentation, 2001).

\section{Structural Savings}

As the majority of the fuel and fuel tank volume is deleted from the spacecraft, the central cylinder, the cylinder that surrounds and supports the fuel tanks, can be removed. In addition, a small amount of other structural savings can be realized because the fuel tanks do not need to be supported through the harsh launch environment. This leads to a structural savings estimate of $17 \mathrm{~kg}$ for both cases.

\section{Launch Vehicle Capacity}

As the AQR 2 spacecraft are launched directly to the staging orbit, there is a mass penalty associated with this case. The launch vehicle mass capacity to staging is $85 \%$ of the capability to the baseline GTO. The total mass capacity for Atlas IIAS to GTO is 3719 $\mathrm{kg}$ and the mass capacity for Delta $2 / 7295$ to GTO is $1869 \mathrm{~kg}$. In addition, using one launch vehicle for two spacecraft requires an additional launch vehicle adapter. The mass of each adapter is assumed to be $100 \mathrm{~kg}$. This leads to a single spacecraft mass capacity of $1480.6 \mathrm{~kg}$ and $1589 \mathrm{~kg}$ for AQR 2a and $2 \mathrm{~b}$, respectively.

\section{Results}

After the components listed above and the launch vehicle capacity are taken into account, a final $\Delta$ mass is found for both $A Q R 2 a$ and $A Q R 2 b$. The calculations for each case are shown in Appendix A.

\footnotetext{
24 This mass savings estimate is from a Space Systems/LORAL employee with access to propulsion system component masses.
} 
The $\Delta$ mass for AQR $2 \mathrm{a}$ is $-5.1 \mathrm{~kg}$. The negative sign indicates that the final AQR $2 \mathrm{a}$ spacecraft mass, before the addition or subtraction of transponders, is greater than the launch vehicle capacity. This occurs because AQR 2a requires two spacecraft on one launch vehicle. The effect of this is discussed further in the limiting factor analysis section. The $\Delta$ mass AQR $2 \mathrm{~b}$ is $1546.4 \mathrm{~kg}$.

\subsubsection{Volume}

The primary volume issue for the AQR 2a spacecraft is whether or not two redesigned spacecraft can fit into one Atlas IIAS. The primary issue for the AQR $2 b$ satellite is how much additional volume will be available for the addition of transponders. To solve these issues, it is necessary to calculate the changes in volume over the baseline satellite. The primary drivers of this change in volume are the fuel and pressurant tanks. These are discussed below.

\section{Fuel Tanks}

The approach used to account for the removal of part of the fuel tank is to delete the standard baseline tank and add in a tank with the capacity to hold the EWSK and NSSK contingency fuel. The volume of the standard fuel tank is found to be $1.9 \mathrm{~m}^{3}$ by multiplying its mass by volume per mass percentage. Using the same method, the volume of the EWSK and NSSK contingency tank is found to be 0.02 and $0.05 \mathrm{~m}^{3}$, for AQR 2a and 2b, respectively.

\section{Pressurant Tanks}

As mentioned previously, the AQR 2a and b spacecraft do not need helium pressurant tanks. Thus, the pressurant tank associated with Program A is deleted from the baseline satellite in the process of forming the AQR 2 spacecraft. This leads to a volume savings of $0.065 \mathrm{~m}^{3}$ for both AQR $2 \mathrm{a}$ and $2 \mathrm{~b}$.

\section{Launch Vehicle Capacity}

The launch vehicle volume capacity is a concern for the AQR 2a spacecraft because two spacecraft are being added to one launch vehicle. Twice the AQR 2 a spacecraft volume is compared to the volume capacity of the Atlas IIAS fairing to determine that it is possible to fit two of the redesigned spacecraft into one fairing. 
The AQR $2 b$ volume is not an issue, from the perspective of launch vehicle capacity, because it is sized to have the same volume as the baseline satellite, which is known to fit into the Atlas IIAS fairing.

\section{Result}

After deleting the baseline fuel tanks and helium pressurant tanks and adding the fuel tank for EWSK and contingency NSSK fuel, the additional volume available for transponders is $1.88 \mathrm{~m}^{3}$ for $\mathrm{AQR} 2 \mathrm{~b}$. It is assumed that no additional volume is available for the AQR 2a spacecraft because the total volume of two AQR 2a satellites is greater than one baseline satellite.

\subsubsection{Spacecraft Cost}

The $\Delta$ (spacecraft cost), neglecting the cost of redesign and the cost of additional transponders, is the cost of the EWSK and contingency fuel tank plus the cost of the tug mechanism, minus the cost of the baseline fuel tank, the NSSK redundant thruster system, the main satellite thruster and the savings associated with the reduced structural needs. For both $A Q R 2 a$ and $2 b$, this leads to a $\Delta$ (spacecraft cost) of $-\$ 0.89 \mathrm{M}$. The negative sign indicates a decrease in cost as compared to the baseline case.

In addition, the cost of redesign is assumed to be $\$ 40 \mathrm{M}$ and $\$ 60 \mathrm{M}$, amortized over 12 spacecraft, for $A Q R 2 a$ and $b$, respectively. This leads to a per spacecraft redesign cost of $\$ 3.33 \mathrm{M}$ and $\$ 5 \mathrm{M}$ for $2 \mathrm{a}$ and $\mathrm{b}$ and total $\Delta$ (spacecraft costs) of $\$ 2.44$ and $\$ 4.11 \mathrm{M}$, respectively.

\subsubsection{Launch Vehicle Cost}

Unlike the $\Delta$ (spacecraft cost), the cost of the launch vehicle per spacecraft differs dramatically for $A Q R 2 a$ and $2 b$. In $A Q R 2 a$, because there are two spacecraft on one launch vehicle, half of the cost of using an Atlas IIAS to launch to the staging orbit is subtracted from the baseline launch cost to get a $\Delta$ (launch cost) per spacecraft of $-\$ 43.9$ $\mathrm{M}$. The negative sign indicates the spacecraft owner saves $\$ 43.9 \mathrm{M}$ for each spacecraft.

In $A Q R 2 b$ the change in launch vehicle cost is calculated by subtracting the baseline launch cost from the cost of using an Atlas IIAS to launch to staging orbit. For both 
$A Q R 2 a$ and $2 \mathrm{~b}$ a $10 \%$ increase in launch vehicle cost is assumed to account for launch vehicle modifications, primarily to the vehicle software, necessary to change the final orbit from GTO to the staging orbit. This is a $\$ 9.8 \mathrm{M}$ increase in launch cost.

\subsubsection{Limiting Factor Analysis}

The limiting factor analysis is used for each case to determine the number of transponders that must be subtracted or can be added to the baseline spacecraft, dependent upon the constraints of the launch vehicle. The two constraints analyzed are mass and volume.

After calculating the volume of the AQR 2a spacecraft and the useable fairing volume for the launch vehicle to confirm that two AQR 2a spacecraft can fit onto an Atlas IIAS, it is found that mass is the limiting factor in determining the number of transponders. In order to fit two spacecraft on an Atlas IIAS, it is necessary to reduce each spacecraft's mass by $5.1 \mathrm{~kg}$. Although this can probably be accomplished without having to remove transponders, for the sake of consistency across the other cases one transponder is removed from the AQR 2 a spacecraft.

The AQR 2b spacecraft also has mass as its limiting factor. However, unlike AQR 2a, the AQR $2 \mathrm{~b}$ spacecraft has an additional available mass of $1546 \mathrm{~kg}$, which corresponds to an additional 56 transponders.

\subsubsection{Revenue and Cost}

The final revenue and cost are found by taking into account the previously mentioned changes as well as those associated with the change in the number of transponders from the baseline case.

\section{Cost}

The total $\Delta$ cost of the spacecraft and launch vehicle is calculated by adding the $\Delta$ costs associated with the spacecraft, additional transponders, and the launch vehicle. In AQR $2 \mathrm{a}$ the change in the cost associated with reducing the number of transponders by one is $-\$ 1.7 \mathrm{M}$. Adding this change to the other cost changes gives a cost decrease of $\$ 43.1 \mathrm{M}$ over the cost of the baseline satellite and launch. This leads to a total spacecraft and launch cost of \$149.4 M. 
In $\mathrm{AQR} 2 \mathrm{~b}$ the $\Delta$ cost associated with increasing the number of transponders on the spacecraft by 56 is $\$ 94.2 \mathrm{M}$. Adding this change to the other cost changes gives a total cost increase of $\$ 108.1 \mathrm{M}$. This leads to a total spacecraft and launch cost of $\$ 300.6 \mathrm{M}$.

\section{Revenue}

The total revenue/year is the baseline revenue/year plus the change in revenue/year. In $\mathrm{AQR} 2 \mathrm{a}$ the decrease in revenue/year is $\$ 2.2 \mathrm{M}$. This leads to a total revenue per year of $\$ 101.6 \mathrm{M}$. In $\mathrm{AQR} 2 \mathrm{~b}$ the increase in revenue/year is $\$ 121.0 \mathrm{M}$, leading to a total revenue per year of $\$ 224.8 \mathrm{M}$.

\subsubsection{Discounted Cash Flow and Options}

Using the revenue and cost numbers discussed above, NPVs for AQR 2a and $b$ are calculated using a DCF analysis. NPVs of $\$ 198.3 \mathrm{M}$ and $\$ 459.6 \mathrm{M}$ are found for AQR $2 \mathrm{a}$ and $\mathrm{b}$, respectively, using revenues of $\$ 101.6$ and $\$ 224.8 \mathrm{M} / \mathrm{yr}$, total costs of $\$ 149.4$ and $\$ 300.6 \mathrm{M}$, and a discount rate of $10 \%$.

In addition to the NPV, the value of the option for life extension is calculated using the method outlined in Section 4.3.11. The option values are found to have present values of $\$ 67.0$ and $\$ 148.7 \mathrm{M}$, for AQR 2a and b, respectively. The NPV and life extension option value are summed for total present values of $\$ 265.3$ and $\$ 608.3 \mathrm{M}$ for AQR $2 \mathrm{a}$ and $\mathrm{b}$.

\subsection{AQR 3: Direct GEO Insertion (DGI) with Tug for North-South Stationkeeping (NSSK)}

\subsubsection{Description}

This case examines the client's perspective of the economic viability of using DGI to launch a redesigned spacecraft directly to GEO and using a tug vehicle to perform its North-South Stationkeeping maneuvers. By using DGI and a tug vehicle for NSSK, a large part of the fuel mass and volume can be reduced. Although fuel mass is reduced, there is a significant mass penalty associated with launching directly to GEO and this volume and mass translate to additional transponders. 


\subsubsection{Mass}

As in the other cases, the serviced spacecraft is formed by subtracting the mass of unnecessary components and adding the mass of required components to the baseline spacecraft mass.

\section{Fuel Tanks}

This case requires substantial changes in the fuel tank mass and volume. As the satellite does not have to perform its own OR or NSSK maneuvers, the fuel tanks associated with these functions are removed. However, the satellite must maintain its ability to do EWSK and have a small amount of contingency fuel onboard for NSSK.

The approach to determining the $\triangle$ mass between the baseline and AQR 3 satellite is to subtract the entire fuel tank, which accounts for OR, NSSK, and EWSK fuel storage, from the baseline satellite and add the fuel tank and fuel associated with EWSK and NSSK contingency. The baseline fuel tank is sized using the method outlined in the fuel tanks discussion of Section 4.3.3.

The reason just the tank volume for OR and NSSK is not simply subtracted, allowing for just the EWSK storage capacity to be left, is because the final AQR 3 spacecraft dry mass is different than the baseline spacecraft. This leads to a different amount of EWSK and NSSK contingency fuel necessary for the life of the spacecraft.

In order to determine the mass of the fuel tanks for EWSK and NSSK contingency, the fuel needed for these maneuvers is calculated. First, the EWSK fuel is calculated. After slight manipulation of the rocket equation, the mass of the EWSK fuel/kg of spacecraft is found to be

$$
\frac{\mathrm{M}_{\text {EWSK fuel }}}{M_{0}}=1-e^{-\frac{\Delta V}{g I_{s p}}}=\frac{0.013 \mathrm{~kg} \text { fuel }}{k g \text { spacecraft }}
$$

where $\Delta \mathrm{V}=(2.0 \mathrm{~m} / \mathrm{s} / \text { year })^{*}($ Lifetime of Spacecraft in Years $)$ and $\mathrm{I}_{\mathrm{sp}}=230 \mathrm{sec}$. This ratio is then multiplied by the mass of the spacecraft to find the total mass of EWSK fuel needed over the lifetime of the satellite. The NSSK fuel is calculated using the same method, except that it is calculated for a single maneuver. This allows the number of NSSK maneuvers supported by the contingency fuel to be varied more easily. For the 
purposes of this analysis, the NSSK contingency fuel is assumed to support two NSSK maneuvers. This leads to a contingency fuel per $\mathrm{kg}$ of spacecraft of

$$
\frac{\mathrm{M}_{\text {NSSK Contingency fuel }}}{M_{0}}=\frac{0.004 \mathrm{~kg} \text { fuel }}{k g \text { spacecraft }}
$$

where a $\Delta V$ of $5.6 \mathrm{~m} / \mathrm{s} / \mathrm{NSSK}$ maneuver and an $\mathrm{I}_{\mathrm{sp}}$ of $268 \mathrm{sec}$ are used.

After using these equations to find the mass of the EWSK and NSSK contingency fuel, the mass of the fuel tanks can be found using the linear relationship found in the fuel tanks discussion in Section 4.3.3. This leads to a mass of $45.1 \mathrm{~kg}$ of fuel and $5.0 \mathrm{~kg}$ for the fuel tank itself. The masses of the fuel and fuel tank are then added to the baseline satellite mass.

\section{Pressurant Tanks}

As the spacecraft in AQR 3 only performs EWSK maneuvers on its own, it does not need a helium pressurant tank. Thus, the pressurant tank associated with Program A is deleted from the baseline satellite in the process of forming the AQR 3 spacecraft. This leads to a mass savings of $23 \mathrm{~kg}$.

\section{Propulsion System}

As the AQR 3 spacecraft does not perform its own orbit raising maneuvers, it does not need a main satellite thruster. Furthermore, it only needs NSSK capabilities for contingency operations. Thus, the main satellite thruster and one level of redundancy for NSSK thrusters are deleted from the baseline satellite. It is not necessary to add any additional propulsion capabilities to the baseline satellite to form the AQR 3 spacecraft. This leads to a mass savings of $20 \mathrm{~kg}$.

\section{Docking and Refueling Mechanisms}

The AQR 3 spacecraft utilizes a tug vehicle to perform its NSSK maneuvers. Thus, it is necessary to add docking capabilities to the baseline satellite. Two sets of identical docking mechanisms are added to the baseline satellite, in order to provide for adequate redundancy. Each of the tug mechanisms has a mass of $16 \mathrm{~kg}$, which leads to a total mass addition of $32 \mathrm{~kg}$. 


\section{Structural Savings}

The majority of the fuel and fuel tank volume is deleted from the spacecraft. Therefore, the central cylinder is removed. In addition, a small amount of other structural savings can be realized because the fuel tanks do not need to be supported through the harsh launch environment. This leads to a structural savings estimate of $17 \mathrm{~kg}$.

\section{Launch Vehicle Capacity}

As the AQR 3 spacecraft is launched directly to GEO, there is a mass penalty associated with this case. The launch vehicle mass capacity to GEO is $76 \%$ of the capability to the baseline GTO. The total mass capacity for Atlas IIAS to GTO is $3719 \mathrm{~kg}$. After accounting for the decreased mass capabilities and the $100 \mathrm{~kg}$ adapter, this leads to a total launch vehicle mass capacity of $2726 \mathrm{~kg}$.

\section{Result}

After the components listed above and the launch vehicle capacity are taken into account, a final $\triangle$ mass is found for AQR 3. The calculations for this case are shown in Appendix A. The $\Delta$ mass for AQR 3 is $1217.9 \mathrm{~kg}$. The positive sign indicates that the AQR 3 spacecraft mass, before the addition or subtraction of transponders, is less than the launch vehicle capacity.

\subsubsection{Volume}

The primary volume issue for the $\mathrm{AQR} 3$ spacecraft is the additional volume available for transponders. To determine this, it is necessary to calculate the change in volume over the baseline satellite. The primary drivers of this change in volume are the fuel tank and the pressurant tank. These are discussed below.

\section{Fuel Tanks}

The approach used to account for the removal of part of the fuel tank is to delete the standard baseline tank and add a tank with the capacity to hold the EWSK and NSSK contingency fuel. The volume of the standard fuel tank is found to be $1.9 \mathrm{~m}^{3}$ by multiplying its mass by the volume per mass percentage. Using the same method, the volume of the EWSK and NSSK contingency tank is found to be $0.01 \mathrm{~m}^{3}$. 


\section{Pressurant Tanks}

As mentioned previously, the AQR 3 spacecraft does not need a helium pressurant tank. Thus, the pressurant tank associated with Program A is deleted from the baseline satellite in the process of forming the AQR 3 spacecraft. This leads to a volume savings of 0.065 $\mathrm{m}^{3}$.

\section{Launch Vehicle Capacity}

The AQR 3 volume is not an issue, from the perspective of launch vehicle capacity, because it is sized to have the same volume as the baseline satellite, which is known to fit into the Atlas IIAS fairing.

\section{Result}

After deleting the baseline fuel tanks and helium pressurant tanks and adding the fuel tank for EWSK and contingency NSSK fuel, the additional volume available for transponders is $1.92 \mathrm{~m}^{3}$ for AQR 3 .

\subsubsection{Spacecraft Cost}

The $\Delta$ (spacecraft cost), neglecting the cost of redesign and the cost of additional transponders, is the cost of the EWSK and contingency fuel tank plus the cost of the tug mechanism, minus the cost of the baseline fuel tank, the NSSK redundant thruster system, the main satellite thruster, and the savings associated with the reduced structural needs. This leads to a $\Delta$ (spacecraft cost) of $-\$ 0.89 \mathrm{M}$. The negative sign indicates a decrease in cost from the baseline case.

In addition, the cost of redesign for $\mathrm{AQR} 3$ is assumed to be $\$ 60 \mathrm{M}$, amortized over 12 spacecraft. This leads to a per spacecraft redesign cost of $\$ 5 \mathrm{M}$ and a total $\Delta$ (spacecraft cost) of $\$ 4.11 \mathrm{M}$.

\subsubsection{Launch Vehicle Cost}

The change in launch vehicle cost is calculated by subtracting the baseline launch cost from the cost of using an Atlas IIAS to launch directly to GEO. This is a $\$ 14.6 \mathrm{M}$ increase in launch cost. 


\subsubsection{Limiting Factor Analysis}

The limiting factor analysis is used for each case to determine the number of transponders that must be subtracted or can be added to the baseline spacecraft, dependent upon the constraints of the launch vehicle. The two constraints analyzed are mass and volume.

The AQR 3 spacecraft has mass as its limiting factor. The AQR 3 spacecraft has an additional available mass of $1218 \mathrm{~kg}$, which corresponds to an additional 44 transponders.

\subsubsection{Revenue and Cost}

The final revenue and cost are found by taking into account the previously mentioned changes as well as those associated with the change in the number of transponders from the baseline case.

\section{Cost}

The change in the cost associated with increasing the number of transponders on the spacecraft by 44 is $\$ 74.0 \mathrm{M}$. Adding this change to the other cost changes gives a total cost increase of $\$ 92.8 \mathrm{M}$. This leads to a total spacecraft and launch cost of $\$ 285.3 \mathrm{M}$.

\section{Revenue}

The total revenue/year is the baseline revenue/year plus the change in revenue/year. The change in revenue/year is proportional to the change in the number of transponders. The increase in revenue/year for $\mathrm{AQR} 3$ is $\$ 95.1 \mathrm{M}$, leading to a total revenue per year of \$198.9 M.

\subsubsection{Discounted Cash Flow and Options}

Using the revenue and cost numbers discussed above, the NPV for AQR 3 is calculated using a DCF analysis. An NPV of $\$ 395.1 \mathrm{M}$ is found with a revenue of $\$ 198.9 \mathrm{M} / \mathrm{yr}$, a total cost of $\$ 285.3 \mathrm{M}$, and a discount rate of $10 \%$.

In addition to the NPV, the value of the option for life extension is calculated using the method outlined in Section 4.3.11. The option value is found to be $\$ 131.5 \mathrm{M}$. The NPV and life extension option value are summed for the total present value of \$526.6 M. 


\subsection{AQR 4: Direct GEO Insertion with Just in Time Refueling}

\subsubsection{Description}

This case examines the client's perspective of the economic viability of launching a redesigned spacecraft directly to GEO and using a servicing vehicle to refuel the satellite between each North-South Stationkeeping maneuver. By using DGI and refueling, a large part of the fuel mass and volume can be reduced. Although fuel and tank mass can be reduced, there is a significant mass penalty associated with launching directly to GEO and this volume and mass translates to additional transponders.

\subsubsection{Mass}

As in the other cases, the serviced spacecraft is formed by subtracting the mass of unnecessary components and adding the mass of required components to the baseline spacecraft mass.

\section{Fuel Tanks}

This case requires substantial changes in the fuel tank mass and volume. As the satellite does not have to perform its own OR maneuver, the fuel tanks associated with this function are removed. The satellite must maintain its ability to do EWSK and NSSK maneuvers. The fuel tanks needed to store the fuel for these maneuvers are refueled between each NSSK maneuver (approximately every 41 days), which makes them much smaller than the standard tanks.

The approach to determining the $\triangle$ mass between the baseline and AQR 4 satellite is to subtract the entire fuel tank, which accounts for OR, NSSK, and EWSK fuel storage, from the baseline satellite and add the fuel tank and fuel associated with one 41-day supply of EWSK and NSSK fuel and contingency fuel. The baseline fuel tank is sized using the method outlined in the fuel tanks discussion of Section 4.3.3.

To determine the mass of the fuel tanks for EWSK and NSSK, the fuel needed for these maneuvers is calculated. First, the EWSK fuel is calculated. After slight manipulation of 
the rocket equation, the mass of the EWSK fuel/kg of spacecraft needed between refuelings is found to be

$$
\frac{\mathrm{M}_{\text {EWSK fuel }}}{M_{0}}=1-e^{-\frac{\Delta V}{g I_{s p}}}=\frac{9.97 E-5 \mathrm{~kg} \text { fuel }}{\mathrm{kg} \text { spacecraft }}
$$

where $\Delta \mathrm{V}=(2.0 \mathrm{~m} / \mathrm{s} / \mathrm{year}) *(41$ days of maneuvers $/ 365$ days/year $)$ and $\mathrm{I}_{\mathrm{sp}}=230 \mathrm{sec}$. This ratio is then multiplied by the mass of the spacecraft to find the total mass of EWSK fuel needed between refuelings.

The NSSK fuel is calculated using the same method, except that it is calculated for only one maneuver. This allows the number of NSSK maneuvers that can be supported between refuelings and by the contingency fuel to be varied more easily. For the purposes of this study, the NSSK contingency fuel is assumed to support two NSSK maneuvers. This leads to a contingency fuel per $\mathrm{kg}$ of spacecraft of

$$
\frac{\mathrm{M}_{\text {NSSK Contingency fuel }}}{M_{0}}=\frac{0.004 \mathrm{~kg} \text { fuel }}{\mathrm{kg} \text { spacecraft }}
$$

and the NSSK fuel between refuelings to be

$$
\frac{\mathrm{M}_{\text {NSSK Contingency fuel }}}{M_{0}}=\frac{0.002 \mathrm{~kg} \text { fuel }}{\mathrm{kg} \text { spacecraft }}
$$

where a $\Delta \mathrm{V}$ of $5.6 \mathrm{~m} / \mathrm{s} / \mathrm{NSSK}$ maneuver and an $\mathrm{I}_{\mathrm{sp}}$ of $268 \mathrm{sec}$ are used.

After using these equations to find the mass of the EWSK and NSSK fuel, the mass of the fuel tanks can be found using the linear relationship found in the fuel tanks discussion in Section 4.3.3. This leads to a mass of $16.8 \mathrm{~kg}$ of fuel and $1.8 \mathrm{~kg}$ for the fuel tank itself. The masses of the fuel and fuel tank are then added to the baseline satellite mass.

\section{Pressurant Tanks}

As with the fuel tanks, it is necessary to re-size the helium pressurant tanks for the AQR 4 spacecraft to account for the helium needed to fill the fuel tanks between each refueling. The approach here is to subtract the baseline satellite pressurant tank, which has a mass of $23 \mathrm{~kg}$, and add the newly sized pressurant tanks. 
The mass of a pressurant tank is proportional to its volume. Thus, the volume of the pressurant tank is calculated and the mass found by linearly scaling it with the mass of the baseline pressurant tank. As helium is used only to pressurize the volume of the fuel tank left empty after each NSSK maneuver, the volume of the helium tank can be found by using the ideal gas law. Therefore, the volume of the helium tank is

$$
\mathrm{V}_{\text {He Tank }}=V_{\text {Re fueled Fuel }}\left(\frac{\mathrm{P}_{\text {Fuel Tank }}}{\mathrm{P}_{\text {He Tank }}}\right)
$$

The mass of the tank is found using

$$
\mathrm{M}_{\text {He Tank }}=M_{\text {Baseline He Tank }}\left(\frac{\mathrm{V}_{\text {He Tank }}}{\mathrm{V}_{\text {Baseline He Tank }}}\right) \text {. }
$$

This leads to a pressurant tank mass of $10.7 \mathrm{~kg}$.

\section{Propulsion System}

As the AQR 4 spacecraft does not perform its own orbit raising maneuvers, it does not need a main satellite thruster. Thus, the main satellite thruster is deleted from the baseline satellite, resulting in a mass savings of $5.0 \mathrm{~kg}$.

\section{Docking and Refueling Mechanisms}

The AQR 4 satellite requires capabilities to dock with and be refueled by a servicing satellite. Therefore, it needs both docking and refueling mechanisms. The redundant docking mechanism has a mass of $32 \mathrm{~kg}$ and the refueling mechanism has a mass of 50 $\mathrm{kg}$. This leads to a mass addition of $82 \mathrm{~kg}$ to the baseline satellite. ${ }^{25}$

\section{Structural Savings}

The majority of the fuel and fuel tank volume is deleted from the spacecraft. Therefore, the central cylinder is removed. In addition, a small amount of other structural savings can be realized because the fuel tanks do not need to be supported through the harsh launch environment. This leads to a structural savings estimate of $17 \mathrm{~kg}$.

\footnotetext{
25 These numbers are from an employee at Space Systems/LORAL, who has experience with satellite servicing studies.
} 


\section{Launch Vehicle Capacity}

As the AQR 4 spacecraft is launched directly to GEO, there is a mass penalty associated with this case. The launch vehicle mass capacity to GEO is $76 \%$ of the capability to the baseline GTO. The total mass capacity for Atlas IIAS to GTO is $3719 \mathrm{~kg}$. After accounting for the reduced launch capability and the mass of a 100-kg launch vehicle adapter, this leads to a total launch vehicle mass capacity of $2726 \mathrm{~kg}$.

\section{Result}

After the components listed above and the launch vehicle capacity are taken into account, a final $\Delta$ mass is found for the AQR 4 spacecraft. The calculations for this case are shown in Appendix A. The $\Delta$ mass for AQR 4 is $1173.6 \mathrm{~kg}$. The positive sign indicates that the final $\mathrm{AQR} 4$ spacecraft mass, before the addition or subtraction of transponders, is less than the launch vehicle capacity.

\subsubsection{Volume}

The primary volume issue for the $\mathrm{AQR} 4$ spacecraft is the volume available for additional transponders. In order to determine this, it is necessary to calculate the change in volume over the baseline satellite. The primary drivers of this change in volume are the fuel tank and the pressurant tank. These are discussed below.

\section{Fuel Tanks}

The approach used to account for the removal of part of the fuel tank is to delete the standard baseline tank and add in a tank with the capacity to hold the a 41 day supply of EWSK and NSSK fuel and NSSK contingency fuel for maneuvers. The volume of the standard fuel tank is found to be $1.9 \mathrm{~m}^{3}$ by multiplying its mass by the volume per mass percentage. Using the same method, the volume of the AQR 4 EWSK and NSSK tank is $0.016 \mathrm{~m}^{3}$.

\section{Pressurant Tanks}

The AQR 4 helium in the pressurant tank is used only to pressurize the volume of the fuel tank left empty after each NSSK maneuver. The equation for the volume of the pressurant tank is presented in the pressurant tanks discussion in Section 4.7.2. The 
deleted baseline pressurant tank has a volume of $0.065 \mathrm{~m}^{3}$, while the volume of the AQR 4 pressurant tank is $0.033 \mathrm{~m}^{3}$.

\section{Launch Vehicle Capacity}

The AQR 4 volume is not an issue, from the perspective of launch vehicle capacity, because it is sized to have the same volume as the baseline satellite, which is known to fit into the Atlas IIAS fairing.

\section{Result}

After deleting the baseline fuel tanks and helium pressurant tanks and adding the resized fuel and pressurant tanks, the additional volume available for transponders is $1.88 \mathrm{~m}^{3}$ for AQR 4.

\subsubsection{Spacecraft Cost}

The $\Delta$ (spacecraft cost), neglecting the cost of redesign and the cost of additional transponders, is the cost of the resized fuel tank plus the cost of the docking and refueling mechanisms, minus the cost of the baseline fuel tank, the NSSK redundant thruster system, the main satellite thruster, and the savings associated with reduced structural needs. This leads to a $\Delta$ (spacecraft cost) of $\$ 5.91 \mathrm{M}$. The positive sign indicates an increase in cost from the baseline case.

The cost of redesign for AQR 4 is assumed to be $\$ 60 \mathrm{M}$, amortized over 12 spacecraft. This leads to a per spacecraft redesign cost of $\$ 5 \mathrm{M}$ for $\mathrm{AQR} 4$ and a total $\Delta$ (spacecraft cost) of \$10.91 M.

\subsubsection{Launch Vehicle Cost}

The change in launch vehicle cost is calculated by subtracting the baseline launch cost from the cost of using an Atlas IIAS to launch directly to GEO. This is a $\$ 14.6 \mathrm{M}$ increase in launch cost. 


\subsubsection{Limiting Factor Analysis}

The limiting factor analysis is used for each case to determine the number of transponders that must be subtracted or can be added to the baseline spacecraft, dependent upon the constraints of the launch vehicle. The two constraints analyzed are mass and volume.

The AQR 4 spacecraft has mass as its limiting factor. The AQR 4 spacecraft has an additional available mass of $1174 \mathrm{~kg}$, which corresponds to an additional 42 transponders.

\subsubsection{Revenue and Cost}

The final revenue and cost are found by taking into account the previously mentioned changes as well as those associated with the change in the number of transponders from the baseline case.

\section{Cost}

The change in the cost associated with increasing the number of transponders on the spacecraft by 42 is $\$ 70.7 \mathrm{M}$. Adding this change to the other cost changes gives a total cost increase of $\$ 96.2 \mathrm{M}$. This leads to a total spacecraft and launch cost of $\$ 288.7 \mathrm{M}$.

\section{Revenue}

The total revenue/year is the baseline revenue/year plus the change in revenue/year. The change in revenue/year is proportional to the change in the number of transponders. The increase in revenue/year for $\mathrm{AQR} 4$ is $\$ 90.8 \mathrm{M}$, leading to a total revenue per year of $\$ 194.5$ M.

\subsubsection{Discounted Cash Flow and Options}

Using the revenue and cost numbers discussed above, the NPV for AQR 4 is calculated using a DCF analysis. An NPV of $\$ 380.6 \mathrm{M}$ is found with a revenue of $\$ 194.5 \mathrm{M} / \mathrm{yr}$, a total cost of $\$ 288.7 \mathrm{M}$, and a discount rate of $10 \%$.

In addition to the NPV, the value of the option for life extension is calculated using the method outlined in Section 4.3.11. The option is found to have a present value of $\$ 128.7$ 
M. The NPV and life extension option value are summed for a total present value of $\$ 509.3 \mathrm{M}$.

\subsection{AQR 5: Direct GEO Insertion with Lower Frequency Refueling}

\subsubsection{Description}

This case examines the client's perspective of the economic viability of using (DGI) to launch a redesigned spacecraft directly to GEO and using a servicing vehicle to refuel the satellite for its North-South stationkeeping maneuvers. The difference between this case and AQR 4 is that the AQR 5 spacecraft is refueled less often. The AQR 5 spacecraft is refueled approximately once a year. It is refueled 12 times over the course of its 15-year lifetime.

This frequency of refueling was chosen because the number of additional transponders that can be added to the spacecraft is a step function of the frequency of refueling. This is because mass is the limiting factor. The slight increases in mass associated with reducing the frequency of refueling by one to two NSSK maneuvers does not necessarily reduce the number of additional transponders that can be added to the spacecraft. Since the number of transponders aboard the spacecraft is a primary driver of cost and revenue, it is optimal to maximize it for a given range of refueling frequency. A serviced spacecraft can support an additional 40 transponders if it is serviced with either $8,9,10$, or 11 NSSK maneuvers between servicings or 39 transponders if it is serviced with either 12,13 , or 14 NSSK maneuvers between servicings. The optimal solution in this range is to have it serviced the least frequently (i.e. 11 NSSK maneuvers between refuelings) with the greatest amount of revenue generation capabilities (i.e. 40 transponders). Having eleven maneuvers between refuelings requires refueling every 451 days, or 12 refuelings over the course of the satellite's 15-year lifetime. The range of 8-14 maneuvers per refueling examined here is approximately one refueling every 1 to 1.5 years. 
This case is important for two reasons. First, the less frequently a servicing vehicle has to visit each of its client spacecraft, the more clients it can have. This reduces the cost of servicing because the on-orbit infrastructure is reduced. Second, the risk associated with servicing can be reduced if the docking and refueling is performed less frequently.

\subsubsection{Mass}

As in the other cases, the serviced spacecraft is formed by subtracting the mass of unnecessary components and adding the mass of required components to the baseline spacecraft mass.

\section{Fuel Tanks}

This case requires substantial changes to the fuel tank mass and volume. As the satellite does not have to perform its own OR maneuvers, the fuel tanks associated with this function are removed. However, the satellite must maintain its ability to do EWSK and NSSK maneuvers. The fuel tanks needed to store the fuel for these maneuvers are replenished after 11 NSSK maneuvers (approximately once every 1.2 years), which makes them much smaller than the standard tanks.

The approach to determining the $\Delta$ mass between the baseline and AQR 5 satellite is to subtract the entire fuel tank, which accounts for OR, NSSK, and EWSK fuel storage, from the baseline satellite and add the fuel tank and fuel associated with a 1.2-year supply of EWSK and NSSK fuel and contingency fuel. The baseline fuel tank is sized using the method outlined in the fuel tanks discussion of Section 4.3.3.

In order to determine the mass of the fuel tanks for EWSK and NSSK, the fuel needed for these maneuvers is calculated. First, the EWSK fuel is calculated. The mass of the EWSK fuel $/ \mathrm{kg}$ of spacecraft needed between refuelings is found to be

$$
\frac{\mathrm{M}_{\text {EWSK fuel }}}{M_{0}}=1-e^{-\frac{\Delta V}{g_{s p p}}}=\frac{0.0011 \mathrm{~kg} \text { fuel }}{\mathrm{kg} \text { spacecraft }}
$$

where $\Delta \mathrm{V}=(2.0 \mathrm{~m} / \mathrm{s} / \mathrm{year}) *(451$ days between refuelings $/ 365$ days $/$ year $)$ and $\mathrm{I}_{\mathrm{sp}}=230$ sec. This ratio is multiplied by the mass of the spacecraft to find the total mass of EWSK fuel needed between refuelings. 
The NSSK fuel is calculated using the same method, but for only one maneuver. This allows the number of NSSK maneuvers that can be supported between refuelings and by the contingency fuel to be varied more easily. For the purposes of this study, the NSSK contingency fuel is assumed to support 2 NSSK maneuvers. This leads to a contingency fuel per $\mathrm{kg}$ of spacecraft of

$$
\frac{\mathrm{M}_{\text {NSSK Contingency fuel }}}{M_{0}}=\frac{0.004 \mathrm{~kg} \quad \text { fuel }}{\mathrm{kg} \text { spacecraft }}
$$

and NSSK fuel between refuelings of

$$
\frac{\mathrm{M}_{\text {NSSK Contingency fuel }}}{M_{0}}=\frac{0.022 \mathrm{~kg} \text { fuel }}{\mathrm{kg} \text { spacecraft }}
$$

using a $\Delta \mathrm{V}$ of $5.6 \mathrm{~m} / \mathrm{s} / \mathrm{NSSK}$ maneuver and an $\mathrm{I}_{\mathrm{sp}}$ of $268 \mathrm{sec}$.

After using these equations to find the mass of the EWSK and NSSK fuel, the mass of the fuel tanks is found using the linear relationship found in the fuel tanks discussion in Section 4.3.3. This leads to a mass of $72.8 \mathrm{~kg}$ of fuel and $8.0 \mathrm{~kg}$ for the fuel tank itself. The masses of the fuel and fuel tank are then added to the baseline satellite mass.

\section{Pressurant Tanks}

It is also necessary to re-size the helium pressurant tanks for the AQR 5 spacecraft to account for the helium needed to fill the fuel tanks between each refueling. The approach here is to subtract the baseline satellite pressurant tank, which has a mass of $23 \mathrm{~kg}$, and add the newly sized pressurant tanks.

The mass of a pressurant tank is proportional to its volume. This allows the volume of the pressurant tank to be calculated and the mass found by linearly scaling it with the mass of the baseline pressurant tank. As the helium is used only to pressurize the volume of the fuel tank left empty after each NSSK maneuver, the volume of the helium tank can be found by using the Ideal Gas Law. Therefore, the volume of the helium tank is

$$
\mathrm{V}_{\text {He Tank }}=V_{\text {Re fulled Fuel }}\left(\frac{\mathrm{P}_{\text {Fuel Tank }}}{\mathrm{P}_{\text {He Tank }}}\right)
$$

The mass of the tank is found using 


$$
\mathrm{M}_{\text {He Tank }}=M_{\text {Baseline He Tank }}\left(\frac{\mathrm{V}_{\text {He Tank }}}{\mathrm{V}_{\text {Baseline He Tank }}}\right)
$$

This leads to a pressurant tank mass of $10.7 \mathrm{~kg}$.

\section{Propulsion System}

As the AQR 5 spacecraft does not perform its own orbit raising maneuvers, it does not need a main satellite thruster. Thus, the main satellite thruster is deleted from the baseline satellite, resulting in a mass savings of $5 \mathrm{~kg}$.

\section{Docking and Refueling Mechanisms}

The AQR 5 satellite requires capabilities to dock with and be refueled by a servicing satellite. Therefore, it needs both docking and refueling mechanisms. The redundant docking mechanism has a mass of $32 \mathrm{~kg}$ and the refueling mechanism has a mass of 50 $\mathrm{kg}$. This leads to a mass addition of $82 \mathrm{~kg}$ to the baseline satellite.

\section{Structural Savings}

The majority of the fuel and fuel tank volume is deleted from the spacecraft. Therefore, the central cylinder can be removed. In addition, a small amount of other structural savings can be realized because the fuel tanks do not need to be supported through the harsh launch environment. This leads to a structural savings estimate of $17 \mathrm{~kg}$.

\section{Launch Vehicle Capacity}

As the AQR 5 spacecraft is launched directly to GEO, there is a mass penalty associated with this case. The launch vehicle mass capacity to GEO is $76 \%$ of the capability to the baseline GTO. The total mass capacity for Atlas IIAS to GTO is $3719 \mathrm{~kg}$. After accounting for the decreased launch capability and the mass of a $100 \mathrm{~kg}$ launch vehicle adapter, this leads to a total launch vehicle mass capacity of $2726 \mathrm{~kg}$.

\section{Result}

After the components listed above and the launch vehicle capacity are taken into account, a final $\Delta$ mass is found for the AQR 5 spacecraft. The calculations for this case are shown in Appendix A. The $\Delta$ mass for AQR 4 is $1111.4 \mathrm{~kg}$. The positive sign indicates that the 
final AQR 5 spacecraft mass, before the addition or subtraction of transponders, is less than the launch vehicle capacity.

\subsubsection{Volume}

The primary volume issue for the AQR 5 spacecraft is the additional volume available for transponders. In order to determine this, it is necessary to calculate the change in volume over the baseline satellite. The primary drivers of this change in volume are the fuel tank and the pressurant tank. These are discussed below.

\section{Fuel Tanks}

The approach used to account for the removal of part of the fuel tank is to delete the standard baseline tank and add in a tank with the capacity to hold the a 451-day supply of EWSK and NSSK fuel and NSSK contingency fuel for 2 maneuvers. The volume of the standard fuel tank is found to be $1.9 \mathrm{~m}^{3}$ by multiplying its mass by the volume per mass percentage. Using the same method, the volume of the AQR 5 EWSK and NSSK tank is found to be $0.071 \mathrm{~m}^{3}$.

\section{Pressurant Tanks}

In AQR 5 the helium in the pressurant tank is used only to pressurize the volume of the fuel tank left empty after each NSSK maneuver. The equation for the volume of the pressurant tank is presented in the pressurant tanks discussion in Section 4.8.2. The deleted baseline pressurant tank has a volume of $0.065 \mathrm{~m}^{3}$, while the volume of the AQR 5 pressurant tank is $0.033 \mathrm{~m}^{3}$.

\section{Launch Vehicle Capacity}

The AQR 5 volume is not an issue, from the perspective of launch vehicle capacity, because it is sized to have the same volume as the baseline satellite, which is known to fit into the Atlas IIAS fairing.

\section{Result}

After deleting the baseline fuel tanks and helium pressurant tanks and adding the resized fuel and pressurant tanks, the additional volume available for transponders is $1.83 \mathrm{~m}^{3}$ for AQR 5. 


\subsubsection{Spacecraft Cost}

The $\Delta$ spacecraft cost, neglecting the cost of redesign and the cost of additional transponders, is the cost of the resized fuel tank plus the cost of the docking and refueling mechanisms, minus the cost of the baseline fuel tank, the NSSK redundant thruster system, the main satellite thruster, and the savings associated with the reduced structural needs. This leads to a $\Delta$ spacecraft cost of $\$ 5.91 \mathrm{M}$. The positive sign indicates an increase in cost from the baseline case.

The cost of redesign for AQR 5 is assumed to be $\$ 60 \mathrm{M}$, amortized over 12 spacecraft. This leads to a per spacecraft redesign cost of $\$ 5 \mathrm{M}$ and a total $\Delta$ (spacecraft cost) of \$10.91 M.

\subsubsection{Launch Vehicle Cost}

The change in launch vehicle cost is calculated by subtracting the baseline launch cost from the cost of using an Atlas IIAS to launch directly to GEO. It is assumed that $15 \%$ increase in launch vehicle cost is incurred to account for launch vehicle modifications, primarily to the vehicle software, necessary to change the final orbit from GTO to GEO. This is a $\$ 14.6 \mathrm{M}$ increase in launch cost.

\subsubsection{Limiting Factor Analysis}

The limiting factor analysis is used for each case to determine the number of transponders that must be subtracted or can be added to the baseline spacecraft, dependent upon the constraints of the launch vehicle. The two constraints analyzed are mass and volume.

The AQR 5 spacecraft has mass as its limiting factor. The AQR 5 spacecraft has an additional available mass of $1111 \mathrm{~kg}$, which corresponds to an additional 40 transponders.

\subsubsection{Revenue and Cost}

The final revenue and cost are found by taking into account the previously mentioned changes as well as those associated with the change in the number of transponders from the baseline case. 


\section{Cost}

The change in the cost associated with increasing the number of transponders on the spacecraft by 40 is $\$ 67.3 \mathrm{M}$. Adding this change to the other cost changes gives a total cost increase of $\$ 102.8 \mathrm{M}$. This leads to a total spacecraft and launch cost of $\$ 285.3 \mathrm{M}$.

\section{Revenue}

The total revenue/year is the baseline revenue/year plus the change in revenue/year. The change in revenue/year is proportional to the change in the number of transponders. The increase in revenue/year for AQR 5 is $\$ 86.5 \mathrm{M}$, leading to a total revenue per year of $\$ 190.2 \mathrm{M}$.

\subsubsection{Discounted Cash Flow and Options}

Using the revenue and cost numbers discussed above, the NPV for AQR 5 is calculated using a DCF analysis. An NPV of $\$ 370.3 \mathrm{M}$ is found with a revenue of $\$ 190.2 \mathrm{M} / \mathrm{yr}$, a total cost of $\$ 285.3 \mathrm{M}$, and a discount rate of $10 \%$.

In addition to the NPV, the value of the option for life extension is calculated using the method outlined in Section 4.3.11. The option value is found to be $\$ 125.9$ M. The NPV and life extension option value are summed for a total present value of $\$ 496.2 \mathrm{M}$.

\subsection{AQR 6: Launch to Staging, Use Tug for Orbit Raising, and Electric Propulsion (EP) for Stationkeeping}

\subsubsection{Description}

This case examines the client's perspective of the economic viability of using servicing and EP on the same spacecraft. The satellite is launched to the staging orbit, where it docks with a tug vehicle that performs its orbit raising maneuvers. The satellite then uses Xenon plasma thrusters to perform all of its own stationkeeping maneuvers. This case is interesting because it takes advantage of the benefits of both servicing and EP. By using a biprop tug vehicle for OR, the spacecraft saves fuel and tank mass and volume. In addition, it is able to get to GEO faster than it would if it were to rely on EP for OR. By 
using EP for stationkeeping, the spacecraft also frees up volume and mass for additional transponders without having to rely on another satellite.

As mentioned previously, there are two means of increasing profit when there is excess volume and mass on a spacecraft. Therefore, the case is broken down into AQR 6a and AQR 6b. AQR 6a examines the effect of reducing launch cost by putting two spacecraft on one launch vehicle. AQR $6 \mathrm{~b}$ examines the effect of filling the excess mass and volume capacity with additional transponders. As the calculations for each case are very similar, they will be presented together throughout this section.

\subsubsection{Mass}

The serviced spacecraft is formed by subtracting the mass of unnecessary components and adding the mass of required components to the baseline spacecraft mass.

\section{Fuel Tanks and Launched Fuel}

This case requires substantial changes in the fuel tank mass and volume. As the satellite does not have to perform its own OR maneuver, the fuel tanks associated with this function are removed. Although the satellite must maintain its ability to do EWSK and NSSK maneuvers, the baseline fuel tanks associated with these functions must be removed to accommodate the added EP fuel tanks.

The approach to determining the $\triangle$ mass between the baseline and AQR 5 satellite is to subtract the entire baseline fuel tank, which accounts for OR, NSSK, and EWSK fuel storage, and add the fuel tank and fuel associated with a lifetime of Xenon fuel for NSSK and EWSK maneuvers. The baseline fuel tank mass is included in the total biprop system mass that is deleted from the baseline satellite. The mass of the EP fuel tanks is included in the mass of the entire EP system that is added to the satellite. This mass is found using the mass of the fuel itself and a ratio of tank mass to fuel mass.

In order to calculate the mass of the Xenon tanks, it is necessary to calculate the mass of the Xenon fuel that is used to fill the fuel tanks. To determine the mass of the fuel for EWSK and NSSK, the rocket equation is used.

The mass of the necessary NSSK and EWSK fuel/kg of spacecraft for the lifetime of the spacecraft is 


$$
M_{\text {Xenon fuel }}=\left(1-e^{-\frac{\Delta V}{g I_{s p}}}\right) M_{\text {Spacecraft }}
$$

where $\Delta \mathrm{V}=(50.0 \mathrm{~m} / \mathrm{s} /$ year $) *(15$ years $)$ and $\mathrm{I}_{\mathrm{sp}}=1507 \mathrm{sec}$. This leads to Xenon fuel masses of $75.0 \mathrm{~kg}$ and $155.1 \mathrm{~kg}$ and fuel tank masses of $9.0 \mathrm{~kg}$ and $18.6 \mathrm{~kg}$ for AQR $6 \mathrm{a}$ and $6 b$, respectively.

\section{Pressurant Tanks}

As the spacecraft in AQR 6 only perform stationkeeping maneuvers on their own, they do not need the baseline helium pressurant tank. Thus, the pressurant tank associated with Program $\mathrm{A}$ is deleted from the baseline satellite in the process of forming the AQR 6 spacecraft. This leads to a mass savings of $23 \mathrm{~kg}$.

\section{Propulsion System}

The AQR 6 spacecraft relies on a tug for orbit raising and electric propulsion for stationkeeping. This eliminates the need for a biprop propulsion system. To form the new propulsion system, the baseline biprop system is deleted and an EP system is added. The baseline biprop system has a mass of $100.9 \mathrm{~kg}$ and the EP system has a mass of $126.4 \mathrm{~kg}$ (Pollard and Janson, 1996).

\section{Docking and Refueling Mechanisms}

As the AQR 6 spacecraft utilizes a tug vehicle to perform its OR maneuvers, it is necessary to add docking capabilities to the baseline satellite. Two sets of identical docking mechanisms are added to the baseline satellite, to provide for adequate redundancy. Each of the docking/tug mechanisms has a mass of $16 \mathrm{~kg}$.

\section{Structural Savings}

The majority of the fuel and fuel tank volume is deleted from the spacecraft. Therefore, a majority of the central cylinder can be removed. In addition, a small amount of other structural savings can be realized because the fuel tanks do not need to be supported through the harsh launch environment. This leads to a structural savings estimate of 12 and $10 \mathrm{~kg}$ for $\mathrm{AQR} 6 \mathrm{a}$ and $\mathrm{b}$, respectively. The slight difference between the structural savings between the two cases arises because the AQR $6 \mathrm{~b}$ spacecraft has a greater mass 
than the AQR 6a spacecraft. This requires the $6 \mathrm{~b}$ spacecraft to carry more Xenon for stationkeeping. This fuel must be supported through the harsh launch environment, which requires slightly more structural support.

\section{Launch Vehicle Capacity}

As the AQR 6 spacecraft are launched directly to the staging orbit, there is a mass penalty associated with this case. The launch vehicle mass capacity to staging is $85 \%$ of the capability to the baseline GTO. The total mass capacity for Atlas IIAS to GTO is 3719 $\mathrm{kg}$.. In addition, using one launch vehicle for two spacecraft requires an additional launch vehicle adapter. The mass of each adapter is assumed to be $100 \mathrm{~kg}$. This leads to a single spacecraft mass capacity of $1480.6 \mathrm{~kg}$ and $3061.2 \mathrm{~kg}$ for AQR 6a and 6b, respectively.

\section{Results}

After the components listed above and the launch vehicle capacity are taken into account, a final $\Delta$ mass is found for both AQR $6 a$ and $b$. The calculations for each case are shown in Appendix A.

The $\Delta$ mass for AQR $6 \mathrm{a}$ is $-172.5 \mathrm{~kg}$. The negative sign indicates that the final AQR $6 \mathrm{a}$ spacecraft mass, before the addition or subtraction of transponders, is greater than the launch vehicle capacity. This occurs because AQR 6a requires two spacecraft on one launch vehicle. The effect of this is discussed further in the limiting factor analysis section. The $\Delta$ mass AQR $6 \mathrm{~b}$ is $1316.4 \mathrm{~kg}$.

\subsubsection{Volume}

The primary volume issue for the AQR 6a spacecraft is whether or not two redesigned spacecraft can fit into one Atlas IIAS. The primary issue for the AQR $6 b$ satellite is the amount of additional volume available for the addition of transponders. It is necessary to calculate the changes in volume over the baseline satellite to solve these issues. The primary drivers of this change in volume are the fuel tanks and the pressurant tank. These are discussed below. 


\section{Fuel Tanks}

The approach used to account for the removal of part of the fuel tank is to delete the standard baseline tank and add a Xenon tank with the capacity to hold a Xenon supply capable of performing all EWSK and NSSK maneuvers over the life of the satellite. The volume of the standard fuel tank is found to be $1.9 \mathrm{~m}^{3}$ by multiplying its mass by the volume per mass percentage. Using the same method with the appropriate volume to mass fraction for a Xenon tank, the volumes of the AQR 6a and b Xenon tanks are found to be 0.044 and $0.090 \mathrm{~m}^{3}$, respectively.

\section{Pressurant Tanks}

As mentioned previously, the AQR 6 spacecraft do not need helium pressurant tanks. Thus, the pressurant tank associated with Program A is deleted from the baseline satellite in the process of forming the AQR 6 spacecraft. This leads to a volume savings of 0.065 $\mathrm{m}^{3}$ for both $6 \mathrm{a}$ and $\mathrm{b}$.

\section{Launch Vehicle Capacity}

The launch vehicle volume capacity is a concern for the AQR 6a spacecraft because two spacecraft are being added to one launch vehicle. Twice the AQR 6a spacecraft volume is compared to the volume capacity of the Atlas IIAS fairing to determine that it is possible to fit two of the redesigned spacecraft into one fairing.

The AQR $6 \mathrm{~b}$ volume is not an issue, from the perspective of launch vehicle capacity, because it is sized to have the same volume as the baseline satellite, which is known to fit into the Atlas IIAS fairing.

\section{Result}

After deleting the baseline fuel tanks and helium pressurant tanks and adding the Xenon fuel tank for EWSK and NSSK fuel, the additional volume available for transponders is $1.77 \mathrm{~m}^{3}$ for $\mathrm{AQR} 6 \mathrm{~b}$. It is assumed that no additional volume is available for the AQR 6a spacecraft because the total volume of two AQR 6a satellites is greater than one baseline satellite. 


\subsubsection{Spacecraft Cost}

The $\Delta$ spacecraft cost, neglecting the cost of redesign and the cost of additional transponders, is the cost of the electric propulsion system plus the cost of the docking mechanisms, minus the cost of the biprop propulsion system and the savings associated with the reduced structural needs. This leads to a $\Delta$ spacecraft cost of $\$ 1.46 \mathrm{M}$ for both AQR 6a and $b$. The positive sign indicates an increase in cost from the baseline case.

In addition, the costs of redesign for $A Q R$ 6a and $b$ are assumed to be $\$ 40 \mathrm{M}$ and $\$ 60 \mathrm{M}$, amortized over 12 spacecraft, respectively. This leads to a per spacecraft redesign cost of $\$ 3.33 \mathrm{M}$ and $\$ 5 \mathrm{M}$ for $6 \mathrm{a}$ and $\mathrm{b}$ and total $\Delta$ (spacecraft costs) of $\$ 4.79$ and $\$ 6.46 \mathrm{M}$, respectively.

\subsubsection{Launch Vehicle Cost}

Unlike the $\Delta$ (spacecraft cost), the cost of the launch vehicle per spacecraft differs dramatically for $A Q R 6 a$ and $6 b$. In AQR 6a, because there are two spacecraft on one launch vehicle, half of the cost of using an Atlas IIAS to launch to the staging orbit is subtracted from the baseline launch cost to get a $\Delta$ (launch cost) per spacecraft of $-\$ 43.9$ M. The negative sign indicates the spacecraft owner saves $\$ 43.9 \mathrm{M}$ for each spacecraft.

In AQR $6 \mathrm{~b}$ the change in launch vehicle cost is calculated by subtracting the baseline launch cost from the cost of using an Atlas IIAS to launch to staging orbit. This is a $\$ 9.8$ $\mathrm{M}$ increase in launch cost.

\subsubsection{Limiting Factor Analysis}

The limiting factor analysis is used for each case to determine the number of transponders that must be subtracted or can be added to the baseline spacecraft, dependent upon the constraints of the launch vehicle. The two constraints analyzed are mass and volume.

After calculating the volume of the AQR 6a spacecraft and the useable fairing volume for the launch vehicle to confirm that two AQR 6a spacecraft can fit onto an Atlas IIAS, it is found that mass is the limiting factor in determining the number of additional transponders. In order to fit two spacecraft on an Atlas IIAS and to accommodate the 
electric propulsion system and fuel, it is necessary to reduce each spacecraft's mass by $172.5 \mathrm{~kg}$. This requires the removal of 7 transponders.

The AQR $6 \mathrm{~b}$ spacecraft also has mass as its limiting factor. However, unlike AQR 6a, the AQR 6b spacecraft has an additional available mass of $1316 \mathrm{~kg}$, which corresponds to an additional 47 transponders.

\subsubsection{Revenue and Cost}

The final revenue and cost are found by taking into account the previously mentioned changes as well as those associated with the change in the number of transponders from the baseline case.

\section{Cost}

The total $\Delta$ cost of the spacecraft and launch vehicle is calculated for each case by adding the $\Delta$ costs associated with the spacecraft, additional transponders, and the launch vehicle. In $A Q R$ 6a the change in the cost associated with reducing the number of transponders by 7 is $-\$ 11.8 \mathrm{M}$. Adding this change to the other cost changes gives a total cost decrease of $\$ 50.9 \mathrm{M}$ over the cost of the baseline satellite and launch. This leads to a total spacecraft and launch cost of \$141.5 M.

In $\mathrm{AQR} 2 \mathrm{~b}$ the $\Delta$ cost associated with increasing the number of transponders on the spacecraft by 47 is $\$ 79.1 \mathrm{M}$. Adding this change to the other cost changes gives a total cost increase of $\$ 95.3 \mathrm{M}$. This leads to a total spacecraft and launch cost of $\$ 287.8 \mathrm{M}$.

\section{Revenue}

The total revenue/year is the baseline revenue/year plus the change in revenue/year. The change in revenue/year is proportional to the change in the number of transponders. For $\mathrm{AQR}$ 6a the decrease in revenue/year is $\$ 15.1 \mathrm{M}$. This leads to a total revenue per year of $\$ 88.6 \mathrm{M}$. For $\mathrm{AQR} 6 \mathrm{~b}$ the increase in revenue/year is $\$ 101.6 \mathrm{M}$, leading to a total revenue per year of $\$ 205.3 \mathrm{M}$.

\subsubsection{Discounted Cash Flow and Options}

Using the revenue and cost numbers discussed above, NPVs for AQR 6a and $b$ are calculated using a DCF analysis. NPVs of $\$ 166.0 \mathrm{M}$ and $\$ 411.8 \mathrm{M}$ are found for AQR 
6a and b respectively, using revenues of $\$ 88.6$ and $\$ 205.3 \mathrm{M} / \mathrm{yr}$, total costs of $\$ 141.5$ and $\$ 287.8 \mathrm{M}$, and a discount rate of $10 \%$.

In addition to the NPV, the value of the option for life extension is calculated using the method outlined in Section 4.3.11. The options are found to have present values of $\$ 58.4$ and \$135.7 M, for AQR 6a and b, respectively. The NPV and life extension option value are summed for total present values of \$224.4 and \$547.5 M.

\subsection{Comp 1: Direct GEO Insertion with Electric Propulsion for Stationkeeping}

\subsubsection{Description}

This case examines the potential client's perspective of the economic viability of using a launch vehicle for Direct GEO Insertion and then performing all stationkeeping maneuvers with electric propulsion (EP). The satellite is launched directly to GEO where it uses Xenon plasma thrusters to perform all of its own stationkeeping maneuvers. This case is interesting because it is the primary source of competition for servicing. Many believe that by using EP, one can gain all of the benefits of servicing (i.e. smaller fuel tanks and thus, more capacity for transponders), without having to rely on another spacecraft. By comparing this case to the AQR cases, the validity of this statement is tested.

While many of the AQR cases examine two distinct cases - one in which the costs are reduced by launching two spacecraft on one launch vehicle and one in which the revenue is increased by increasing the number of transponders on the spacecraft - only one case is examined here: the increased revenue case. This is because the reduced launch capacity to GEO eliminates the possibility of launching two spacecraft on one launch vehicle, without significantly reducing the number of revenue generating transponders onboard such that the case becomes uneconomical.

\subsubsection{Mass}

The spacecraft is formed by subtracting the mass of unnecessary components and adding the mass of required components to the baseline spacecraft mass. 


\section{Fuel Tanks and Launched Fuel}

This case requires substantial changes in the fuel tank mass and volume. As the satellite does not have to perform its own OR maneuver, the fuel tanks associated with this function are removed. Although the satellite must maintain its ability to do EWSK and NSSK maneuvers, the baseline fuel tanks associated with these functions must be removed to accommodate the added EP fuel tanks.

The approach to determining the $\Delta$ mass between the baseline and Comp 1 satellite is to subtract the entire baseline fuel tank, which accounts for OR, NSSK, and EWSK fuel storage, and add the fuel tank and fuel associated with a lifetime of Xenon fuel for NSSK and EWSK maneuvers. The baseline fuel tank mass is included in the total biprop system mass that is deleted from the baseline satellite. The mass of the EP fuel tanks is included in the mass of the entire EP system that is added to the satellite. This mass is found using the mass of the fuel itself and a ratio of tank mass to fuel mass.

In order to calculate the mass of the Xenon tanks, it is necessary to calculate the mass of the Xenon fuel that is used to fill the fuel tanks. The rocket equation is used to determine the mass of the fuel for EWSK and NSSK. The mass of the NSSK and EWSK fuel $/ \mathrm{kg}$ of spacecraft needed for the lifetime of the spacecraft is

$$
M_{\text {Xenon fuel }}=\left(1-e^{-\frac{\Delta V}{g I_{s p}}}\right) M_{\text {Spacecraft }}=138 \mathrm{~kg}
$$

where $\Delta \mathrm{V}=(50.0 \mathrm{~m} / \mathrm{s} /$ year $) *(15$ years $)$ and $\mathrm{I}_{\mathrm{sp}}=1507 \mathrm{sec}$. This leads to a Xenon fuel tank mass of $16.6 \mathrm{~kg}$.

\section{Pressurant Tanks}

As the Comp 1 spacecraft, only performs stationkeeping maneuvers on its own, it does not need the baseline helium pressurant tank. Thus, the pressurant tank associated with Program A is deleted from the baseline satellite in the process of forming the Comp 1 spacecraft. This leads to a mass savings of $23 \mathrm{~kg}$. 


\section{Propulsion System}

The Comp 1 spacecraft relies on the launch vehicle to get to GEO and electric propulsion for stationkeeping. This eliminates the need for a biprop propulsion system. To form the new propulsion system, the baseline biprop system is deleted and an EP system is added. The baseline biprop system has a mass of $100.9 \mathrm{~kg}$ and the EP system has a mass of $126.4 \mathrm{~kg}$.

\section{Structural Savings}

The baseline fuel tank volume is deleted from the spacecraft. A much smaller Xenon tank replaces it. Therefore, a majority of the central cylinder can be removed. In addition, a small amount of other structural savings can be realized because the fuel tanks do not need to be supported through the harsh launch environment. This leads to a structural savings estimate of $11 \mathrm{~kg}$.

\section{Launch Vehicle Capacity}

As the Comp 1 spacecraft is launched directly to GEO, there is a mass penalty associated with this case. The launch vehicle mass capacity to GEO is $76 \%$ of the capability to the baseline GTO. The total mass capacity for Atlas IIAS to GTO is $3719 \mathrm{~kg}$. After accounting for the decreased launch capability and the mass of a 100-kg launch vehicle adapter, this leads to a total launch vehicle mass capacity of $2726 \mathrm{~kg}$.

\section{Results}

After the components listed above and the launch vehicle capacity are taken into account, a final $\Delta$ mass is found for Comp 1 . The calculations for each case are shown in Appendix A. The $\Delta$ mass for Comp 1 is $1094 \mathrm{~kg}$.

\subsubsection{Volume}

The primary volume issue for the Comp 1 spacecraft is the additional volume available for transponders. To determine this volume, it is necessary to calculate the change in volume over the baseline satellite. The primary drivers of this change are the substitution of the biprop fuel tanks with a smaller Xenon tank and the deletion of the pressurant tank. These are discussed below. 


\section{Fuel Tanks}

The approach used to account for the removal of part of the fuel tank is to delete the standard baseline tank and add a Xenon tank with the capacity to hold a Xenon supply capable of sustaining all EWSK and NSSK maneuvers over the life of the satellite. The volume of the standard fuel tank is found to be $1.9 \mathrm{~m}^{3}$ by multiplying its mass by the volume per mass percentage. Using the same method with the appropriate volume to mass fraction for a Xenon tank, the volume of the Comp 1 Xenon tank is found to be $0.080 \mathrm{~m}^{3}$.

\section{Pressurant Tanks}

As mentioned previously, the Comp 1 spacecraft does not need helium pressurant tanks. Thus, the pressurant tank associated with Program A is deleted from the baseline satellite in the process of forming the Comp 1 spacecraft. This leads to a volume savings of 0.065 $\mathrm{m}^{3}$.

\section{Launch Vehicle Capacity}

The Comp 1 spacecraft volume is not an issue, from the perspective of launch vehicle capacity, because it is sized to have the same volume as the baseline satellite, which is known to fit into the Atlas IIAS fairing.

\section{Result}

After deleting the baseline fuel tanks and helium pressurant tanks and adding the Xenon fuel tank for EWSK and NSSK fuel, the additional volume available for transponders is $1.88 \mathrm{~m}^{3}$ for Comp 1 .

\subsubsection{Spacecraft Cost}

The $\Delta$ spacecraft cost, neglecting the cost of redesign and the cost of additional transponders, is the cost of the electric propulsion system minus the cost of the biprop propulsion system and the savings associated with the reduced structural needs. This leads to a $\Delta$ spacecraft cost of $\$ 0.96 \mathrm{M}$. The positive sign indicates an increase in cost from the baseline case. 
The cost of redesign for Comp 1 is assumed to be $\$ 60 \mathrm{M}$, amortized over 12 spacecraft. This leads to a per spacecraft redesign cost of $\$ 5 \mathrm{M}$ and a total $\Delta$ (spacecraft cost) of $\$ 5.96 \mathrm{M}$.

\subsubsection{Launch Vehicle Cost}

The change in launch vehicle cost is calculated by subtracting the baseline launch cost from the cost of using an Atlas IIAS to launch directly to GEO. It is assumed that $15 \%$ increase in launch vehicle cost is incurred to account for launch vehicle modifications, primarily to the vehicle software, necessary to change the final orbit from GTO to GEO. This is a $\$ 14.6 \mathrm{M}$ increase in launch cost.

\subsubsection{Limiting Factor Analysis}

The limiting factor analysis is used for each case to determine the number of transponders that must be subtracted or can be added to the baseline spacecraft, dependent upon the constraints of the launch vehicle. The two constraints that are analyzed are mass and volume.

The Comp 1 spacecraft also has mass as its limiting factor. The Comp 1 spacecraft has an additional available mass of $1094 \mathrm{~kg}$, which corresponds to an additional 39 transponders.

\subsubsection{Revenue and Cost}

The final revenue and cost are found by taking into account the previously mentioned changes as well as those associated with the change in the number of transponders from the baseline case.

\section{Cost}

The total $\Delta$ cost of the spacecraft and launch vehicle is calculated for each case by adding the $\Delta$ costs associated with the spacecraft, additional transponders, and the launch vehicle. In Comp 1 the change in cost associated with increasing the number of transponders by 39 is $\$ 65.6 \mathrm{M}$. Adding this change to the other cost changes gives a total cost increase of $\$ 86.2 \mathrm{M}$ over the cost of the baseline satellite and launch. This leads to a total spacecraft and launch cost of $\$ 278.7 \mathrm{M}$. 


\section{Revenue}

The total revenue/year is the baseline revenue/year plus the change in revenue/year. The change in revenue/year is proportional to the change in the number of transponders. In Comp 1 the increase in revenue/year is $\$ 84.3 \mathrm{M}$. This leads to a total revenue per year of $\$ 188.1 \mathrm{M}$.

\subsubsection{Discounted Cash Flow and Options}

Using the revenue and cost numbers discussed above, the NPV for Comp 1 is calculated using a DCF analysis. An NPV of $\$ 368.2 \mathrm{M}$ is found with a revenue of $\$ 188.1 \mathrm{M} / \mathrm{yr}$, a total cost of $\$ 278.7 \mathrm{M}$, and a discount rate of $10 \%$.

\subsection{Comp 2: Launch to Staging Orbit and use Electric Propulsion for Orbit Raising and Stationkeeping}

\subsubsection{Description}

This case examines the potential client's perspective of the economic viability of an entirely EP-based propulsion system. The satellite is launched to the staging orbit where it then uses Xenon plasma thrusters to perform all of its own orbit raising and stationkeeping maneuvers. This case is interesting because it is the other primary source of competition for servicing. Many believe that by using EP, one can gain all of the benefits of servicing (i.e. smaller fuel tanks and thus, more capacity for transponders), without having to rely on another spacecraft. By comparing this case to the $A Q R$ cases, the validity of this statement is tested. This case is also useful in determining whether it is more economical to launch to staging and use EP for OR and SK or to use DGI and EP only for stationkeeping, as in Comp 1.

Another aspect of this case is that using electric propulsion is a much slower means of orbit raising. This is important because the revenue stream for this case needs to be shifted by the additional time it takes to get to GEO. 


\subsubsection{Mass}

The spacecraft is formed by subtracting the mass of unnecessary components and adding the mass of needed components to the baseline spacecraft mass.

\section{Fuel Tanks and Launched Fuel}

This case requires substantial changes in the fuel tank mass and volume. Although the satellite performs all of its own orbit raising and stationkeeping maneuvers, the baseline fuel tanks associated with these functions must be removed to accommodate added EP fuel tanks.

The approach to determining the $\Delta$ mass between the baseline and Comp 2 satellites is to subtract the entire baseline fuel tank, which accounts for OR, NSSK, and EWSK fuel storage, and add the fuel tank and fuel associated with a lifetime of Xenon fuel for orbit raising, NSSK, and EWSK maneuvers. The baseline fuel tank mass is included in the total biprop system mass that is deleted from the baseline satellite. The mass of the EP fuel tanks is included in the mass of the entire EP system that is added to the satellite. This mass is found using the mass of the fuel itself and a ratio of tank mass to fuel mass.

In order to calculate the mass of the Xenon tanks, it is necessary to calculate the mass of the Xenon fuel that is used to fill the fuel tanks. The rocket equation is used to determine the mass of the fuel for orbit raising, EWSK, and NSSK. The mass of the total fuel $/ \mathrm{kg}$ of spacecraft needed for the lifetime of the spacecraft is

$$
M_{\text {Xenon fuel }}=\left(1-e^{-\frac{\Delta V}{g I_{s p}}}\right) M_{\text {Spacecraft }}=577.3 \mathrm{~kg}
$$

where $\Delta \mathrm{V}=1800 \mathrm{~m} / \mathrm{s}+(50.0 \mathrm{~m} / \mathrm{s} / \text { year })^{*}(15$ years $)$ and $\mathrm{I}_{\mathrm{sp}}=1507 \mathrm{sec}$. This leads to a Xenon fuel tank mass of $69.3 \mathrm{~kg}$.

\section{Pressurant Tanks}

As the Comp 2 spacecraft, performs all of its maneuvers using its electric propulsion system a separate pressurant tank is not necessary. Thus, the pressurant tank associated with Program A is deleted from the baseline satellite in the process of forming the Comp 2 spacecraft. This leads to a mass savings of $23 \mathrm{~kg}$. 


\section{Propulsion System}

The Comp 2 spacecraft relies solely on electric propulsion for its maneuvers. This eliminates the need for a biprop propulsion system. To form the new propulsion system, the baseline biprop system is deleted and an EP system is added. The baseline biprop system has a mass of $100.9 \mathrm{~kg}$ and the EP system has a mass of $126.4 \mathrm{~kg}$.

\section{Structural Savings}

The baseline fuel tank volume is deleted from the spacecraft. A much smaller Xenon tank replaces it. Therefore, a majority of the central cylinder can be removed. In addition, a small amount of other structural savings can be realized because the fuel tanks do not need to be supported through the harsh launch environment. This leads to a structural savings estimate of $11 \mathrm{~kg}$.

\section{Launch Vehicle Capacity}

As the Comp 2 spacecraft is launched directly to the staging orbit, there is a mass penalty associated with this case. The launch vehicle mass capacity to staging is $85 \%$ of the capability to the baseline GTO (Turner, Ref. 2, 2001). The total mass capacity for Atlas IIAS to GTO after accounting for the $100 \mathrm{~kg}$ adapter is $3619 \mathrm{~kg}$.

\section{Results}

After the components listed above and the launch vehicle capacity are taken into account, a final $\Delta$ mass is found for Comp 2. The calculations for each case are shown in Appendix A. The $\Delta$ mass for Comp 1 is $1481 \mathrm{~kg}$.

\subsubsection{Volume}

The primary volume issue for the Comp 1 spacecraft is the amount of additional volume available for transponders. In order to determine this volume, it is necessary to calculate the change in volume over the baseline satellite. The primary drivers of this change in volume are the substitution of the biprop fuel tank with smaller a smaller Xenon tank and the deletion of the pressurant tank. These are discussed below. 


\section{Fuel Tanks}

The approach used to account for the removal of a portion of the fuel tank is to delete the standard baseline tank and add a Xenon tank with the capacity to hold a Xenon supply capable of sustaining all orbit raising, EWSK, and NSSK maneuvers over the life of the satellite. The volume of the standard fuel tank is found to be $1.9 \mathrm{~m}^{3}$ by multiplying its mass by the volume per mass percentage. Using the same method with the appropriate volume to mass fraction for a Xenon tank, the volume of the Comp 2 Xenon tank is found to be $0.34 \mathrm{~m}^{3}$.

\section{Pressurant Tanks}

As mentioned previously, the Comp 2 spacecraft does not need helium pressurant tanks. Thus, the pressurant tank associated with Program A is deleted from the baseline satellite in the process of forming the Comp 2 spacecraft. This leads to a volume savings of 0.065 $\mathrm{m}^{3}$.

\section{Launch Vehicle Capacity}

The Comp 2 volume is not an issue, from the perspective of launch vehicle capacity, because it is sized to have the same volume as the baseline satellite, which is known to fit into the Atlas IIAS fairing.

\section{Result}

After deleting the baseline fuel tanks and helium pressurant tanks and adding the Xenon fuel tank for the orbit raising, EWSK, and NSSK fuel, the additional volume available for transponders is $1.05 \mathrm{~m}^{3}$ for Comp 2 .

\subsubsection{Spacecraft Cost}

The $\Delta$ spacecraft cost, neglecting the cost of redesign and the cost of additional transponders, is the cost of the electric propulsion system minus the cost of the biprop propulsion system and the savings associated with the reduced structural needs. This leads to a $\Delta$ spacecraft cost of $\$ 0.96 \mathrm{M}$. The positive sign indicates an increase in cost from the baseline case. 
Additionally, the cost of redesign for Comp 2 is assumed to be $\$ 60 \mathrm{M}$, amortized over 12 spacecraft. This leads to a per spacecraft redesign cost of $\$ 5 \mathrm{M}$ and a total $\Delta$ (spacecraft cost) of $\$ 5.96 \mathrm{M}$.

\subsubsection{Launch Vehicle Cost}

There is no change in the launch vehicle cost from the baseline case because the Comp 2 spacecraft uses the Atlas IIAS launch vehicle to launch to GTO.

\subsubsection{Limiting Factor Analysis}

The limiting factor analysis is used for each case to determine the number of transponders that must be subtracted or can be added to the baseline spacecraft, dependent upon the constraints of the launch vehicle. The two constraints analyzed are mass and volume.

The Comp 2 spacecraft has volume as its limiting factor. The Comp 2 spacecraft has an additional available volume of $1.05 \mathrm{~m}^{3}$, which corresponds to 46 additional transponders.

\subsubsection{Revenue and Cost}

The final revenue and cost are found by taking into account the previously mentioned changes as well as those associated with the change in the number of transponders from the baseline case.

\section{Cost}

The total $\Delta$ cost of the spacecraft and launch vehicle is calculated for each case by adding the $\Delta$ costs associated with the spacecraft, additional transponders, and the launch vehicle. In Comp 2 the change in the cost associated with increasing the number of transponders by 46 is $\$ 77.4 \mathrm{M}$. Adding this change to the other cost changes gives a total cost increase of $\$ 83.3 \mathrm{M}$ over the cost of the baseline satellite and launch. This leads to a total spacecraft and launch cost of $\$ 275.8 \mathrm{M}$.

\section{Revenue}

The total revenue/year is the baseline revenue/year plus the change in revenue/year. The change in revenue/year is proportional to the change in the number of transponders. In 
Comp 2 the increase in revenue/year is $\$ 99.4 \mathrm{M}$. This leads to a total revenue per year of $\$ 203.2 \mathrm{M}$.

\subsubsection{Discounted Cash Flow and Options}

Using the revenues and costs discussed above, the NPV for Comp 2 is calculated using a DCF analysis. As using EP for orbit raising increases the time to GEO by approximately 30 days, the satellite will be unable to provide services to its clients until 30 days after a satellite that utilizes biprop for orbit raising. Thus, its revenues must be shifted by 30 days to account for the increased time for orbit raising. Taking the shifted revenues into account, an NPV of $\$ 408.7 \mathrm{M}$ is found with a revenue of $\$ 203.2 \mathrm{M} / \mathrm{yr}$, a total cost of $\$ 275.8 \mathrm{M}$, and a discount rate of $10 \%$.

\subsection{Results and Discussion}

Six Aquarius servicing cases and two competition cases are analyzed and presented individually. This section illustrates the results of each of the cases and how they compare to each other. 
Table 4-3 Value of Satellite Servicing from Client Perspective

\begin{tabular}{|c|c|c|c|c|c|c|}
\hline Case \# & \begin{tabular}{|c} 
Redesign \\
Costs \\
(amortized over \\
$12 \mathrm{~s} / \mathrm{c})$ \\
$(\$ \mathrm{M})$
\end{tabular} & $\begin{array}{c}\text { Total S/C } \\
\text { and Launch } \\
\text { Cost before } \\
\text { Servicing } \\
\text { Charges } \\
(\$ M)\end{array}$ & $\begin{array}{c}\text { Revenue/yr } \\
\text { (\$M) }\end{array}$ & $\begin{array}{c}\text { Project } \\
\text { NPV after } \\
\text { redesign } \\
\text { costs (\$M) }\end{array}$ & $\begin{array}{c}\text { Life } \\
\text { Extension } \\
\text { Option } \\
\text { Value (\$M) }\end{array}$ & $\begin{array}{c}\text { Total } \\
\text { Present } \\
\text { Value }(\$ M)\end{array}$ \\
\hline Baseline & 0 & 192.5 & 103.8 & 179.3 & - & 179.3 \\
\hline AQR 1a & 40 & 146.3 & 90.78 & 169.4 & 59.9 & 229.3 \\
\hline AQR 2a & 40 & 149.4 & 101.6 & 198.3 & 67.0 & 265.3 \\
\hline AQR 2b & 60 & 300.6 & 224.8 & 459.6 & 148.7 & 608.3 \\
\hline AQR 3 & 60 & 280.3 & 198.9 & 395.1 & 131.5 & 526.6 \\
\hline AQR 4 & 60 & 288.7 & 194.5 & 380.6 & 128.7 & 509.3 \\
\hline AQR 5 & 60 & 285.3 & 190.2 & 370.3 & 125.9 & 496.2 \\
\hline AQR 6a & 40 & 141.5 & 88.6 & 166 & 58.4 & 224.4 \\
\hline AQR 6b & 60 & 287.8 & 205.3 & 411.8 & 135.7 & 547.5 \\
\hline AQR 7 & 60 & 350.5 & 181.6 & 307.8 & - & 307.8 \\
\hline Comp 1 & 60 & 278.7 & 188.1 & 368.2 & - & 368.2 \\
\hline Comp 2 & 60 & 275.8 & 203.2 & 412.8 & - & 408.7 \\
\hline
\end{tabular}

It is interesting to note that before servicing costs are taken into account, all of the servicing cases, as well as the competition cases, have higher total present values than the baseline case. This indicates that both satellite servicing and electric propulsion provide economic benefit to the client spacecraft.

Based solely on the information provided in Table 4-3, the best means of servicing a satellite, from the client's economic perspective, is AQR $2 b$, utilizing a tug vehicle for both OR and NSSK. This allows for the most additional transponders to be added to the 
spacecraft for the least cost, providing the highest total present value for the client spacecraft. ${ }^{26}$

In order to determine the client's preferred method of servicing, it is necessary to also consider her confidence in the reliability of servicing (See Section 4.3.1). Although AQR $2 \mathrm{~b}$ provides the greatest economic value for the client, it requires the client to be completely reliant upon the provider. $\mathrm{AQR} 4,5$, and $6 \mathrm{~b}$ have lower total present values, but they require less reliance on the provider. Depending on the technical risk aversion of the client, the client may prefer these cases to AQR $2 b$.

Although the cases that present the greatest economic benefit to the client are servicing cases, as the client determines her confidence in the reliability of servicing, she may decide to forgo the added benefit of servicing over the competition cases because EP may provide less risk. As the risk of EP versus servicing is out of the scope of this thesis, it will not be considered throughout the remainder of the servicing analysis. However, EP remains the primary competition for servicing, and as such its client NPV will be considered in the process of determining the amount a client is willing to pay for servicing.

The numbers presented above do not take into account the value of the option to relocate the spacecraft over a more profitable market. This value is believed to be comparable the value of life extension. However, as the value of the option to relocate is dependent upon many factors that are specific to a particular spacecraft mission (see Section 4.3.12), it is not included in the generalized analysis presented in this thesis.

\subsection{Conclusions}

This chapter presents both the economic and technical issues associated with potential satellite servicing configurations. The client's economic value of servicing and the technical issues associated with each are presented.

A potential client, needs to consider both the economic and technological aspects of each of the cases before determining which servicing configuration, if any, is best for her

\footnotetext{
${ }^{26}$ This is before the costs of servicing are taken into account. These costs are discussed in the next chapter.
} 
purposes. The client also needs to consider electric propulsion as a means of receiving the benefits that satellite servicing provides, namely increasing mass and volume available to transponders, with potentially less technical risk.

From a purely economic view, the client spacecraft gains more by utilizing satellite servicing than electric propulsion. Of the servicing cases, the largest NPV is earned by building the AQR $2 b$ spacecraft, a satellite that utilizes a tug for both orbit raising and North-South stationkeeping.

From a purely technical standpoint, there are many trades that need to be performed to determine the risk associated with each servicing approach. The technical feasibility of frequent dockings, tugs, and refuelings needs to be investigated. However, it is reasonable to assume that the client prefers to minimize her dependence on the servicing spacecraft. Given more information about the technical risk associated with each servicing approach, a potential client can determine which servicing case is "best" based upon the risk she is willing to incur to maximize her NPV.

The analysis presented in this chapter indicates the viability of the satellite servicing market from the client's perspective. Based on current knowledge, by considering both the technical and economic issues, best configuration for the client spacecraft is AQR 5 . This case has a significant increase in NPV over the baseline case and has minimal interactions with the servicing spacecraft. (less than one time per year). 



\section{Chapter 5. Satellite Servicing: Provider Value Analysis}

\subsection{Introduction}

This chapter uses the information and insights gained from the satellite servicing analysis in Chapter 4 to demonstrate the process of estimating the value of satellite servicing to the provider. The estimated values for each case are then compared to determine the proper servicing architecture.

First, the purpose and approach of this analysis is presented. Next, the analysis itself, including a sensitivity analysis, is shown. Finally, conclusions about the viability of the servicing market and the proper system architecture are presented.

\subsection{Purpose}

It is important to recognize that the market must provide economic benefit to the provider as well as to the client. If it does not, the provider will be unwilling to provide satellite servicing to the client. While the focus of many valuations is the cost of providing the service or product, this thesis highlights the importance of valuing the technology from the client's perspective, as well. However, in doing so, it is important that the provider's costs are not neglected. Otherwise, the valuation would only examine one side of the 
coin, just as current valuations do when they only consider the problem from the provider's (or cost) perspective.

The purpose of this analysis is to find:

- The revenue stream to the provider from satellite servicing based upon the client's economic benefit

- The necessary architecture to support this servicing approach

- The costs associated with each servicing approach

- The viability of the satellite servicing market using the revenue stream, necessary architectures and their associated costs based on value to the provider

System architecture selection is driven by both economics and technical analysis. If one lets the system architecture be driven by the technical aspects alone, it is very likely that the final product will be technically impressive, but unable to fulfill the needs of the market it is attempting to capture. On the other hand, if the architecture is driven solely by economics it is possible that the system will run into technical obstacles along the way that were not properly accounted for up front. Thus, in addition to the economic analysis, the technological risks associated with each servicing case and its associated servicing architecture are considered in the final analysis.

\subsection{Approach}

To determine the economic viability of a market, it is necessary to consider the price a client is willing to pay and the costs associated with the necessary architecture to provide the service. These are discussed below, followed by a discussion of the discounted cash flow analysis used to determine the servicing provider's NPV for each servicing architecture.

\subsubsection{Estimating Potential Revenues from Servicing}

Estimating the servicing market's potential revenues requires estimating the price a client is willing to pay to have her/his satellite serviced and the number of satellites that will use 
this service. This price estimate is made by comparing the competition cases to the baseline case to determine which provides the most economic benefit to the client. This information is used to find the difference between the NPV of the servicing case and the client's next best option, which determines the value servicing provides. A portion of this value is the price that a client is willing to pay to have her/his satellite serviced. To estimate the number of satellites whose owners will be willing to pay for servicing requires using market forecasts for GEO commercial satellites.

In addition to the revenue estimations, it is very important to determine when the provider is actually paid for its services (i.e. the time at which the revenue is generated). Due to the time value of money, the timing of the revenue has a significant impact on the final NPV calculation.

\subsubsection{Estimating Cost of Servicing}

In addition to estimating the revenue stream a given architecture, it is important to investigate its associated costs. A set of estimates for the cost of each architecture is presented below. These values are not meant to be accurate representations of the costs associated with each architecture, rather they are included to demonstrate how they are to be used with the revenues to determine the provider's NPV.

To estimate the cost of servicing for each case, one must investigate the cost of the onorbit architecture, its associated launch costs, the costs of fuel delivery, and its operations costs. The servicing cases are broken into three classes based upon their on-orbit architectures to facilitate the estimation of spacecraft and launch costs. The fuel delivery costs are calculated as a function of the amount of fuel necessary for each serviced satellite and when the fuel is delivered. The operations costs are estimated to be a percentage of the revenues generated by the provider.

The development costs, in terms of the refueling and docking interfaces, are assumed to be small. Although there will inevitably be some development costs incurred by a servicing provider, much of the technology is currently being developed by groups funded by government agencies (see Section 2.4.2). 
In addition to the actual cost amounts, it is very important to determine when each of the costs is incurred. Due to the effects of discounting, the timing of each of the costs has a significant impact on the final NPV calculation. Each of the costs and their associated timing is discussed below in Section 5.4.2.

\subsubsection{Discounted Cash Flow to Determine Provider's NPV}

After the revenues and costs associated with each case are determined, a discounted cash flow analysis is used to calculate the servicing provider's NPV for each case. The DCF analysis is based upon industry driven assumptions of the financing of current aerospace projects. $^{27}$ It includes taxes, depreciation, debt and equity financing, interest expenses, and satellite operating costs. The model is set up such that the primary inputs are servicing revenue and servicing costs (including architecture and fuel delivery costs) with their appropriate timing. The model's primary output is an NPV based on a given discount rate. For the purposes of this analysis the following assumptions are made:

- Corporate tax rate $=40 \%$

- Loan amount $=80 \%$ of cash needed

- Capital Expenditure depreciated over lifetime of servicing architecture (15 years)

- Interest rate $=10 \%$

- Spacecraft and launch costs are paid evenly during two years before launch date

- $\quad$ Operating Expenses $=10 \%$ of gross revenue generated per year

- Discount rate $=10 \%$ (this number is varied to show sensitivity to cost of capital)

\subsubsection{Break-Even Analysis}

In addition to the DCF analysis, a break-even analysis is performed to determine the maximum servicing architecture cost for which the servicing provider would still be willing to take on the project. This occurs when NPV is slightly positive. The analysis is

\footnotetext{
27 The DCF analysis is a modified version of an analysis used by the finance group at Space Systems/LORAL to value satellite projects.
} 
performed using the DCF analysis and the assumptions listed above. The servicing architecture cost is varied until an NPV of approximately zero is found.

This analysis is important because the cost estimates used in the DCF analysis are just that - estimates. The break-even analysis allows the servicing provider to make her own estimate of the cost of a servicing architecture and to compare it with the break-even cost. It also allows the potential provider to better understand what her cost constraints are.

\subsection{Analysis}

\subsubsection{Estimating Potential Revenues from Servicing}

Estimating the potential revenues generated from servicing requires an analysis of the "value" servicing provides to its clients as well as the number of spacecraft that a given architecture will service. The majority of the client value analysis is presented in Chapter 4. The information from that analysis is used to determine how it affects the servicing provider in terms of how much a client is willing to pay to have her spacecraft serviced. To round out the revenue picture, an estimate is made of the number of spacecraft that will use servicing.

\section{Price Estimation for Servicing}

To determine the price a client is willing to pay requires deciding what her next best option is. As addressed previously, the competition cases are economically more attractive to a potential client than the current spacecraft design (the baseline case). Thus, the "value" of servicing is the difference between the economic value (i.e. NPV) of each of the servicing cases and Comp 1.

Unfortunately, comparing the total NPVs against each other does not directly take into consideration the difference in costs incurred to generate those NPVs. Nor does it directly factor in the fact that there are different numbers of transponders on board each case's spacecraft in each case. A means of solving both of these issues is to normalize each case's NPV with its respective number of transponders and then to compare the NPV difference per transponder. This is the approach taken to estimate the price a client would be willing to pay for servicing. 
Using the total present values presented in Section 4.12, the differences in total present value (standard NPV + value of option to extend spacecraft life) between each of the cases and Comp 1, are calculated and then multiplied by the number of transponders for that respective case.

$$
\begin{gathered}
\text { "Value" of Servicing } \\
\text { for Case } X
\end{gathered}=\left(\begin{array}{c}
\frac{\text { Case X Total Discounted Value }}{\# \text { Transponders in Case } X} \\
\frac{\text { Comp } 1 \text { Total Discounted Value }}{\# \text { Transponders in Comp } 1}
\end{array}\right)\left(\begin{array}{c}
\# \text { Transponders } \\
\text { in Case X }
\end{array}\right)
$$

It is important to recognize that the price a client is willing to pay for servicing is between \$0 and the "value" calculated above. For the purposes of this study it is assumed that this value is split evenly between the provider and the client. Thus, the NPV of the price a client is willing to pay to have his/her satellite serviced is $50 \%$ of the above-calculated value.

In addition to the NPV of the price of servicing, it is important to determine when the provider generates these revenues. Each case is considered individually to determine when it is serviced does this, as that is the approximate time the client would pay for the servicing.

For $A Q R$ 1a and $A Q R$ 6a and b, the only interaction with the provider occurs right after launch (i.e. year 1). Thus, the client will pay the entire price in year 1. AQR 2a and b has a significant portion of the servicing performed in year 1. The rest of the servicing is spread evenly throughout the spacecraft's lifetime. This leads to $50 \%$ of the NPV being paid in year 1 and $50 \%$ being spread out over the life of the spacecraft. AQR 3, 4, and 5 have servicing needs that are evenly distributed throughout their lifetimes, which leads to evenly distributing the NPV of the servicing price over the spacecraft lifetime. In addition to deciding when the provider is paid in each case, the time value of money is taken into account when the actual servicing price is calculated by forecasting the price over the 15-year lifetime and discounting it back to year 0 to confirm that the discounted value of the revenues generated by the provider match the initial NPV of the servicing price. The calculated servicing NPVs and their respective servicing price and timing are shown in Table 5-1. 
Table 5-1 Price Client is Willing to Pay for Servicing

\begin{tabular}{|c|c|c|c|}
\hline Servicing Case & $\begin{array}{c}\text { NPV of Servicing Price } \\
\text { over S/C Lifetime }\end{array}$ & $\begin{array}{c}\text { Servicing Price } \\
\mathbf{( \$ M} \text {, after } \\
\text { discounting) }\end{array}$ & When is Servicer Paid? \\
\hline AQR 1a & 14.62 & 14.62 & $100 \%$ Year 1 \\
\hline AQR 2a & 23.28 & $13.30,1.66 / \mathrm{yr}$ & $50 \%$ Year 1, 50\% over lifetime \\
\hline AQR 2b & 88.28 & $50.45,6.3058 / \mathrm{yr}$ & $50 \%$ Year $1,50 \%$ over lifetime \\
\hline AQR 3 & 69.85 & $9.18 / \mathrm{yr}$ & $100 \%$ over lifetime \\
\hline AQR 4 & 64.97 & $8.537 / \mathrm{yr}$ & $100 \%$ over lifetime \\
\hline AQR 5 & 62.11 & $8.161 / \mathrm{yr}$ & $100 \%$ over lifetime \\
\hline AQR 6a & 14.02 & 14.02 & $100 \%$ Year 1 \\
\hline AQR 6b & 74.73 & 74.73 & $100 \%$ Year 1 \\
\hline
\end{tabular}

\section{Market Forecasts}

After determining the price the client is willing to pay and its associated timing, it is necessary to determine how many spacecraft will utilize servicing. It is estimated that one servicing architecture can service 12 spacecraft (Turner, Ref. 4, 2001). Although each of the different servicing architectures may be able to support a different number of clients, it is assumed that each architecture services 12 spacecraft. To determine if the commercial GEO market be able to accommodate this number of spacecraft in the future, it is necessary to look at market forecasts.

Many firms and agencies attempt to forecast the number of satellites that will be launched over the next few years. A forecast of an average of approximately 30 spacecraft launched per year until 2010 (Commercial Space Transportation Forecasts, 2001) fits well with both historical data ("Space Launch Roundup," 2001) and predicted future satellite demands. These predictions indicate that there is a viable market for 12 serviced GEO spacecraft, from a quantity standpoint.

In addition to simply predicting the number of spacecraft being launched, the forecasts examine the trends in satellite mass. Over the past several years, as indicated by their masses, satellites have been increasing in size. It appears that this trend is not going to change in the foreseeable future (Space Launch Roundup," 2001). The upward trend in satellite mass indicates an increase in satellite capacity and coverage over time 
(Commercial Space Transportation Forecasts, 2001). Along with the number of spacecraft forecast, the trends towards increasing capability and coverage indicate that the commercial GEO market should absorb 12 serviced spacecraft, with an increased number of transponders, without a decrease in the amount of revenue each transponder generates. However, it is unlikely that this market would, in the near-term, support two on-orbit architectures (24 serviced spacecraft), as that would amount to $80 \%$ of the entire predicted GEO market.

\subsubsection{Estimating Costs of Servicing}

The costs of servicing include the on-orbit architecture and its launch costs, fuel delivery costs, and operations costs. Each of these is discussed below.

\section{Architecture costs}

To estimate the cost of each architecture, the architectures associated with each AQR case are separated into groups that have the same on-orbit architectures. The groups, their on-orbit architectures, and estimated costs are presented in Table 5-2.

Table 5-2 Architecture Groups and Associated Cost Estimates

\begin{tabular}{|c|c|c|c|}
\hline $\begin{array}{l}\text { Architecture } \\
\text { Group }\end{array}$ & Cases & $\begin{array}{c}\text { Basic Servicing } \\
\text { Architecture }\end{array}$ & Cost Estimate \\
\hline $\mathrm{A}$ & $\begin{array}{l}\text { AQR } 1 \\
\text { AQR } 6\end{array}$ & $\begin{array}{c}1 \text { Orbital Transfer Vehicle } \\
\text { between LEO and Staging, } \\
1 \text { Servicing Spacecraft and } \\
\text { Fuel Storage Facility at } \\
\text { Staging Orbit }\end{array}$ & $\begin{array}{c}\text { Spacecraft: } \$ 100 \mathrm{M} \\
\text { Storage Facility: } \$ 5 \mathrm{M} \\
\text { Launches: } \$ 94.5 \mathrm{M} \\
\text { Total: } \$ 199 \mathrm{M}\end{array}$ \\
\hline B & AQR 2 & $\begin{array}{c}\text { 1 Orbital Transfer Vehicle } \\
\text { between LEO and Staging, } \\
1 \text { Orbital Transfer Vehicle } \\
\text { between Staging and GEO, } \\
1 \text { Servicing Spacecraft and } \\
\text { Fuel Storage Facility at } \\
\text { Both Staging and GEO } \\
\text { Orbits }\end{array}$ & $\begin{array}{c}\text { Spacecraft: } \$ 200 \mathrm{M} \\
\text { Storage Facilities: } \$ 10 \mathrm{M} \\
\text { Launches: } \$ 144 \mathrm{M} \\
\text { Total: } \$ 354 \mathrm{M}\end{array}$ \\
\hline $\mathrm{C}$ & $\begin{array}{l}\text { AQR } 3 \\
\text { AQR } 4 \\
\text { AQR } 5\end{array}$ & $\begin{array}{c}\text { 1 Orbital Transfer Vehicle } \\
\text { between LEO and GEO, } \\
1 \text { Servicing Spacecraft and } \\
\text { Fuel Storage Facility at } \\
\text { GEO Orbit }\end{array}$ & $\begin{array}{c}\text { Spacecraft: } \$ 100 \mathrm{M} \\
\text { Storage Facility: } \$ 5 \mathrm{M} \\
\text { Launches: } \$ 94.5 \mathrm{M} \\
\text { Total: } \$ 199 \mathrm{M}\end{array}$ \\
\hline
\end{tabular}


The costs listed in Table 5-2 are derived from the following estimations:

- Orbital transfer vehicle (OTV): $\$ 50 \mathrm{M}$

- Servicing spacecraft: $\$ 50 \mathrm{M}$

- On orbit fuel storage facility: $\$ 5 \mathrm{M}$

- Launch on Delta 2: $\$ 45 \mathrm{M}$ to LEO, $\$ 49.5 \mathrm{M}$ to staging

The orbital transfer vehicle and servicing spacecraft costs are estimated by assuming they are small satellites, on the order of $500 \mathrm{~kg}$ (Turner, Ref. 4, 2001), with medium complexity (Bearden, 2001). A single on-orbit storage facility is assumed to be $\$ 5 \mathrm{M} .^{28}$ The launch cost for a Delta 2 to LEO is $\$ 45 \mathrm{M}$ (Isakowitz, 1999). A $10 \%$ increase in cost is assumed for launches to the staging orbit. The total launch costs are derived using the above launch costs and the number of launches as illustrated in Table 5-3.

Table 5-3 Necessary Launches for Each Servicing Architecture Group

\begin{tabular}{|c|c|c|}
\hline $\begin{array}{l}\text { Architecture } \\
\text { Group }\end{array}$ & Launches & $\begin{array}{c}\text { Spacecraft and Storage Facilities on } \\
\text { Launch }\end{array}$ \\
\hline \multirow[t]{2}{*}{$\mathrm{A}$} & 1 Delta 2 to LEO & LEO to Staging OTV \\
\hline & 1 Delta 2 to Staging & $\begin{array}{c}\text { Staging Servicing Spacecraft and Fuel } \\
\text { Storage Facility }\end{array}$ \\
\hline \multirow[t]{3}{*}{ B } & 1 Delta 2 to LEO & $\begin{array}{c}\text { LEO to Staging OTV and Staging Fuel } \\
\text { Storage Facility (tugged to Staging by OTV) }\end{array}$ \\
\hline & 1 Delta 2 to Staging & $\begin{array}{c}\text { Staging to GEO OTV and Staging Servicing } \\
\text { Spacecraft }\end{array}$ \\
\hline & 1 Delta 2 to Staging & $\begin{array}{c}\text { GEO Servicing Vehicle and Fuel Storage } \\
\text { Facility }\end{array}$ \\
\hline \multirow[t]{2}{*}{$\mathrm{C}$} & 1 Delta 2 to LEO & LEO to GEO OTV \\
\hline & 1 Delta 2 to Staging & $\begin{array}{l}\text { GEO Servicing Spacecraft and Fuel Storage } \\
\text { Facility }\end{array}$ \\
\hline
\end{tabular}

For a visual representation of each architecture, refer to Figure 5-1,

\footnotetext{
${ }^{28}$ This estimate is from a Space Systems/LORAL employee who works on possible servicing architectures.
} 
Figure 5-2, and Figure 5-3. From these illustrations, it is clear why architecture B is so much more expensive than the other two architectures. 


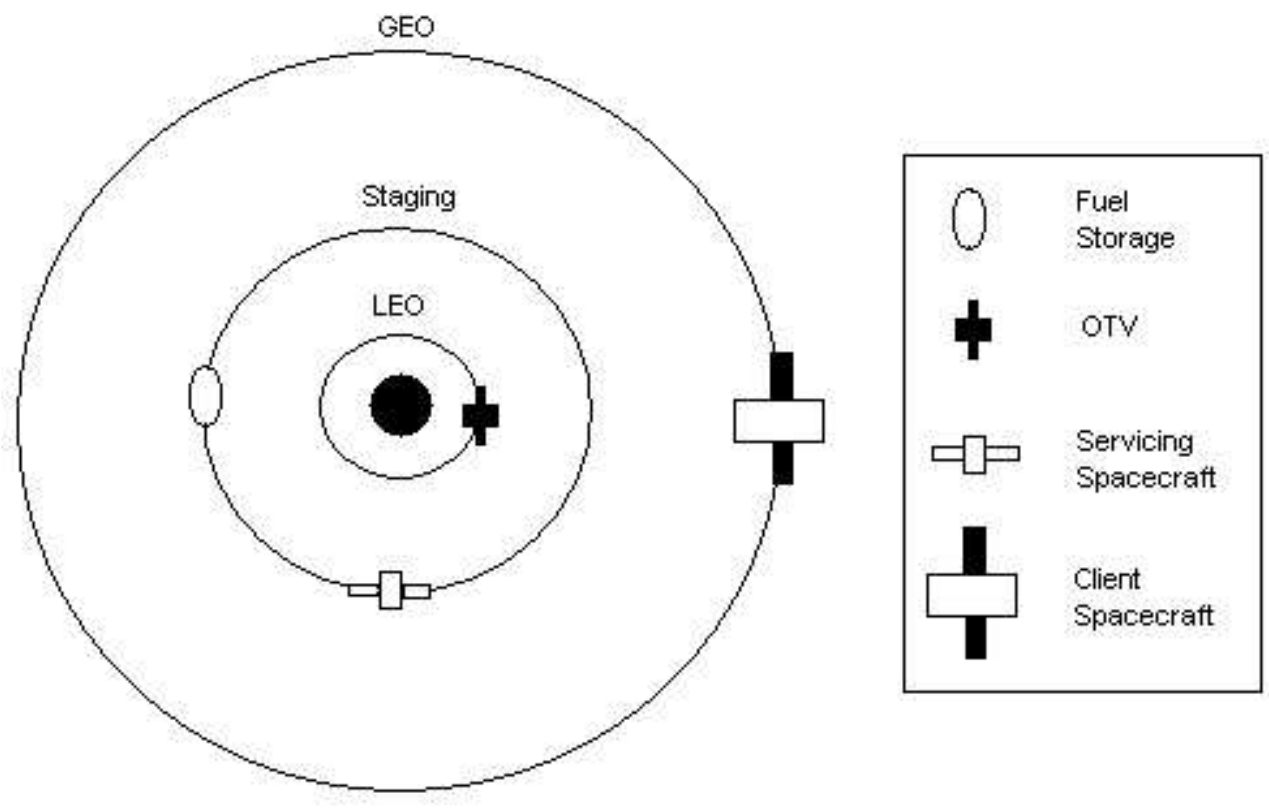

Figure 5-1 Servicing Architecture A

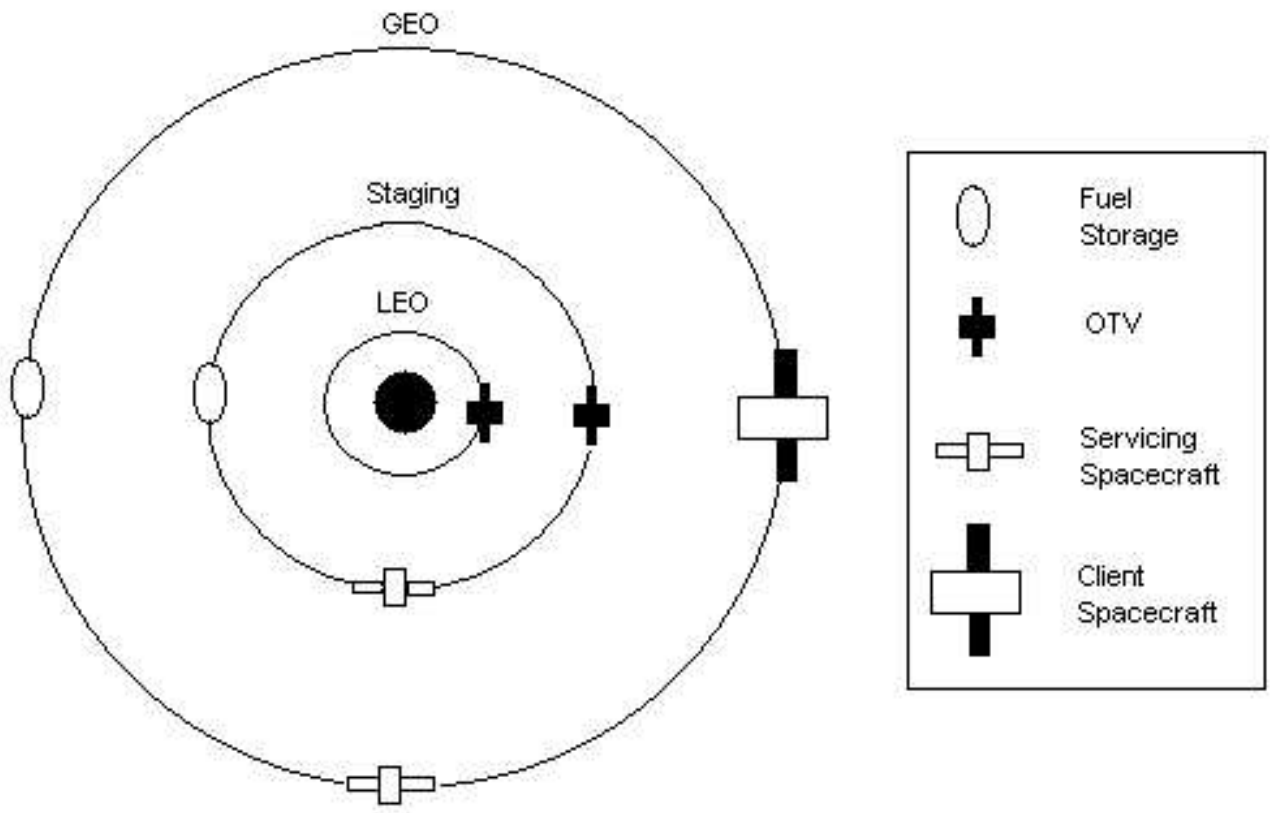

Figure 5-2 Servicing Architecture B 


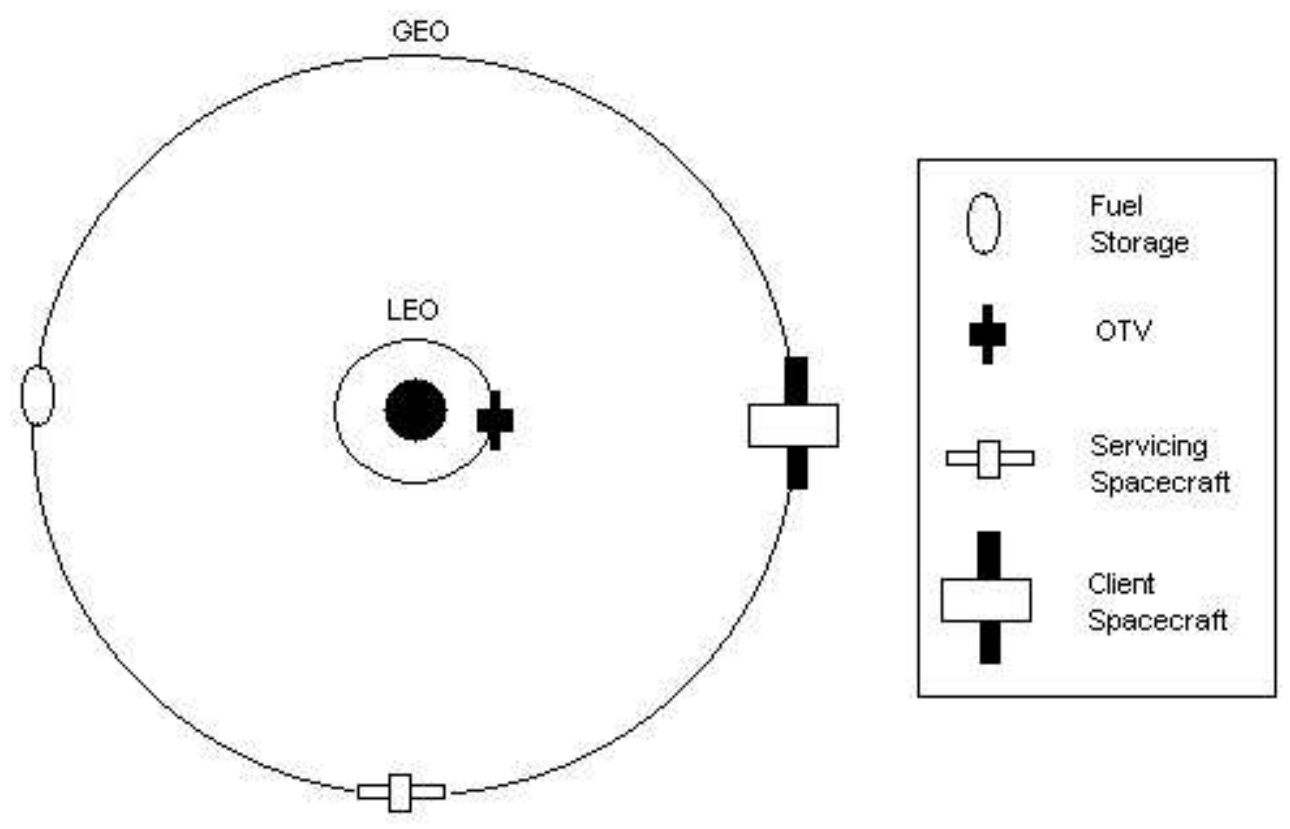

\section{Figure 5-3 Servicing Architecture C}

\section{Fuel delivery costs}

The cost of fuel delivery for each case is estimated by finding the amount of fuel a single client spacecraft needs in each orbit (i.e. staging or GEO), multiplying this number by the number of spacecraft serviced by one servicing architecture, and adding the cost of fuel for the servicing vehicle to transfer between the client spacecraft. As discussed above, it is very important to know the timing of the fuel delivery costs. These times are included in the analysis, as well.

The mass of the fuel a single client spacecraft (and its tug vehicle if appropriate) needs over the course of its lifetime is calculated using the equations presented in the fuel tanks discussion of Section 4.3.3.

The cost of fuel delivery is assumed to be $\$ 13.6 \mathrm{~K} / \mathrm{kg}$ to staging and $\$ 30 \mathrm{~K} / \mathrm{kg}$ to GEO. ${ }^{29}$ Although these numbers are derived assuming the use of a low cost launch vehicle, such as Aquarius, the cost to GEO is not unreasonable compared to current launch costs. ${ }^{30}$

\footnotetext{
${ }^{29}$ These numbers were generated using software developed by Space Systems/Loral.

${ }^{30}$ The specific cost to GEO for the Atlas IIAS vehicle is $\$ 34 \mathrm{~K}$.
} 
After calculating the mass of the fuel to be delivered and multiplying it by the delivery costs, the following costs are found (See Table 5-4).

\section{Table 5-4 Fuel Delivery Costs For Client Maneuvers Per Client Spacecraft}

\begin{tabular}{|c|c|c|}
\hline Servicing Case & $\begin{array}{c}\text { Cost of Client Fuel } \\
\text { Delivery in Year 1 } \mathbf{( \$ M )}\end{array}$ & $\begin{array}{c}\text { Cost of Client Fuel } \\
\text { Delivery per Year } \\
\text { over Lifetime (\$M) }\end{array}$ \\
\hline AQR 1a & 37.6 & - \\
\hline AQR 2a & 33.4 & 1.0 \\
\hline AQR 2b & 60.1 & 1.9 \\
\hline AQR 3 & 1.7 & 1.7 \\
\hline AQR 4 & 1.5 & 1.5 \\
\hline AQR 5 & 1.5 & 1.5 \\
\hline AQR 6a & 32.4 & - \\
\hline AQR 6b & 58.3 & - \\
\hline
\end{tabular}

In addition to the fuel costs listed above, there are fuel delivery costs associated with the fuel necessary to maneuver the servicing spacecraft between the client satellites. These support costs are estimated using the mass of the fuel needed per client spacecraft for the provider to maneuver between and dock with the spacecraft and the fuel storage facility, multiplied by the delivery cost/kg of propellant at GEO.

The fuel necessary to maneuver between and dock with the client and the fuel storage facility is $4.35 \mathrm{~kg} / \mathrm{client}$ per docking. ${ }^{31}$ This number is multiplied by the number of docking operations per year to determine the total mass of the fuel needed for the servicer. The fuel mass multiplied by the fuel delivery cost, leads to support costs below. (See Table 5-5)

\footnotetext{
${ }^{31}$ This number is found by estimating the total annual fuel used by a servicing vehicle that performs all NSSK maneuvers for a spacecraft and services 12 spacecraft and dividing it by 12 spacecraft and the number of times it has to dock with one spacecraft/year.
} 
Table 5-5 Fuel Delivery Costs for Support Maneuvers Per Client Spacecraft

\begin{tabular}{|c|c|c|}
\hline Servicing Case & $\begin{array}{c}\text { Cost of Support Fuel } \\
\text { Delivery in Year } 1 \text { (\$M) }\end{array}$ & $\begin{array}{c}\text { Cost of Support } \\
\text { Fuel Delivery per } \\
\text { Year over Lifetime } \\
(\$ M)\end{array}$ \\
\hline AQR 1a & 0.1 & - \\
\hline AQR 2a & 1.3 & 1.2 \\
\hline AQR 2b & 1.2 & 1.2 \\
\hline AQR 3 & 1.2 & 1.2 \\
\hline AQR 4 & 1.2 & 1.2 \\
\hline AQR 5 & 0.1 & 0.1 \\
\hline AQR 6a & 0.1 & - \\
\hline AQR 6b & 0.1 & - \\
\hline
\end{tabular}

The costs in Table 5-4 and Table 5-5 are summed to get the total fuel delivery cost per client spacecraft.

\section{Operations costs}

The operations costs for each case are estimated to be $10 \%$ of gross revenues. Since the revenues scale with the number of clients as well as the value of the revenues for each client, the operations costs scale with these variables as well. This is expected from operations costs because the more clients the provider has to service and the more valuable each client is, the more expensive operations costs will be.

\subsubsection{Discounted Cash Flow to Determine Provider's NPV}

After calculating the above revenues and costs, these numbers are used as inputs in a DCF analysis. The purpose of this analysis is to determine the servicing provider's NPV. Using a discount rate of $10 \%$ and the other assumptions presented in Section 5.3.3, the following NPVs are found. (See Table 5-6) 
Table 5-6 Servicing Provider's NPV

\begin{tabular}{|c|c|c|c|c|c|}
\hline $\begin{array}{l}\text { Servicing } \\
\text { Case }\end{array}$ & $\begin{array}{c}\text { Servicing Revenue } \\
\text { Year 1, Year 2-15 } \\
\text { (Table 5-1) } \\
(\$ M)\end{array}$ & $\begin{array}{c}\text { Cost of } \\
\text { Servicing } \\
\text { Architecture } \\
\text { (Table 5-2) } \\
\text { (\$M) }\end{array}$ & $\begin{array}{l}\text { Cost of Fuel } \\
\text { Delivery in } \\
\text { Year } 1 \\
\text { (Tables 5-4 } \\
\text { and 5-5) } \\
\text { (\$M) }\end{array}$ & $\begin{array}{l}\text { Cost of Fuel } \\
\text { Delivery per } \\
\text { Year over } \\
\text { Lifetime } \\
\text { (Tables 5-4 } \\
\text { and 5-5) } \\
\text { (\$M) }\end{array}$ & $\begin{array}{c}\text { Provider } \\
\text { NPV } \\
\text { (\$M) }\end{array}$ \\
\hline AQR 1a & $14.6,0 / \mathrm{yr}$ & 199.5 & 37.7 & - & -397.7 \\
\hline AQR 2a & $13.3,1.7 / \mathrm{yr}$ & 354.0 & 34.6 & 2.2 & -561.2 \\
\hline AQR 2b & $50.5,6.3 / \mathrm{yr}$ & 354.0 & 61.3 & 3.0 & -280.4 \\
\hline AQR 3 & $9.2,9.2 / \mathrm{yr}$ & 199.5 & 2.9 & 2.9 & 95.7 \\
\hline AQR 4 & $8.5,8.5 / \mathrm{yr}$ & 199.5 & 2.7 & 2.7 & 80.3 \\
\hline AQR 5 & $8.2,8.2 / \mathrm{yr}$ & 199.5 & 1.6 & 1.6 & 109.0 \\
\hline AQR 6a & $14.2,0 / \mathrm{yr}$ & 199.5 & 32.4 & - & -355.4 \\
\hline AQR 6b & $74.7,0 / \mathrm{yr}$ & 199.5 & 58.3 & - & -103.5 \\
\hline
\end{tabular}

\subsubsection{Break-Even Analysis}

The break-even analysis is performed using the revenue and fuel delivery costs listed in Table 5-6. The following servicing architecture costs are the break-even costs for the servicing provider. (See Table 5-7)

Table 5-7 Break-Even Analysis for Servicing Provider

\begin{tabular}{|c|c|}
\hline Servicing Case & $\begin{array}{c}\text { Architecture cost for } \\
\text { which } \mathbf{~ N P V ~ = ~ 0 ~} \\
\mathbf{( \$ M )}\end{array}$ \\
\hline AQR 1a & negative \\
\hline AQR 2a & negative \\
\hline AQR 2b & 22.4 \\
\hline AQR 3 & 358.8 \\
\hline AQR 4 & 332.9 \\
\hline AQR 5 & 381 \\
\hline AQR 6a & negative \\
\hline AQR 6b & 62.3 \\
\hline
\end{tabular}

As shown in Table 5-7, some of the break-even costs are negative. This indicates that there is no cost at which the servicing provider would take on this project, for the given set of assumptions. In fact, an outside party would actually have to give the provider the on orbit architecture and then pay the provider more than the estimated revenues to make this an economically attractive project for the provider. 


\subsubsection{Sensitivity Analysis}

A sensitivity analysis is presented here to demonstrate the sensitivity of the servicing provider's NPV to each of the inputs into the DCF analysis, as well as to the discount rate. The baseline inputs for the sensitivity analysis are presented in Table 5-6.

\section{Revenues: Percentage of client's NPV client is willing to pay for servicing}

The owner of the client spacecraft is willing to pay between $\$ 0$ and the total "value" servicing provides them to the servicing provider for their spacecraft to be serviced. In the DCF analysis presented above, it is assumed that the client is willing to pay the provider $50 \%$ of their increased value due to servicing. To determine the sensitivity of the DCF analysis to this percentage, each of the cases is examined using $25 \%$ and $75 \%$. The results are presented in Figure 5-4.

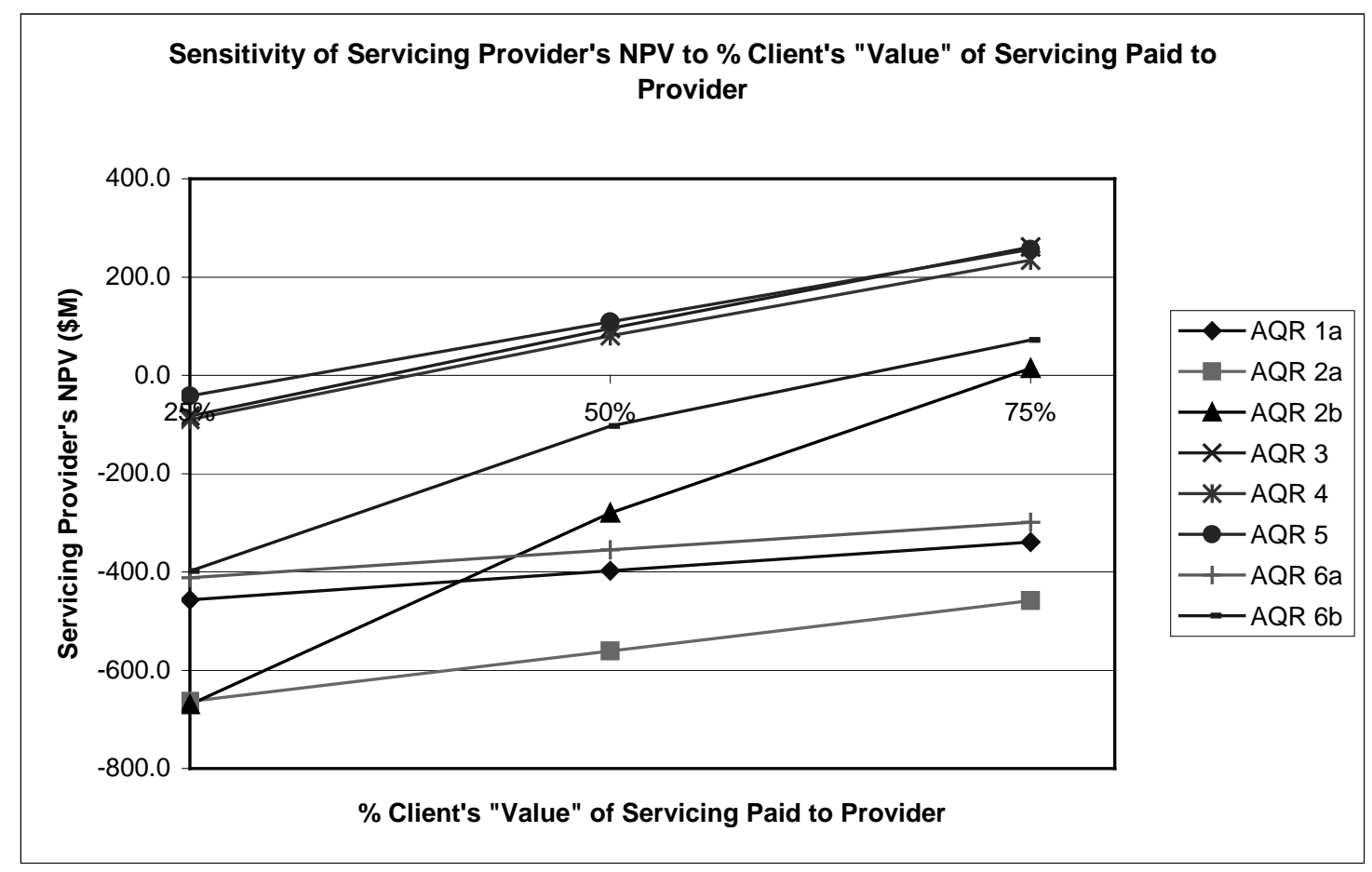

Figure 5-4 Sensitivity of Provider's NPV to \% of Client's Value Paid to Provider 
Figure 5-4 demonstrates several important issues. First, no cases are viable if the servicing revenue is less than $32 \%$ of the client's value for servicing. Second, two of the cases, AQR $2 \mathrm{~b}$ and $6 \mathrm{~b}$, become economically viable at $73 \%$ and $65 \%$, respectively.

It is also interesting to note that $\mathrm{AQR} 1 \mathrm{a}, 2 \mathrm{a}$, and $6 \mathrm{a}$ are the only cases that are linear. (Although the other cases may appear linear on this graph, they are slightly non-linear.) This occurs because the provider revenue associated with each of these cases is much lower than any of the other cases. Due to the effects of taxes in the DCF model, cases that generate very little revenue do not receive as much of a tax shield because they have fewer revenues to shield. This effect leads to non-linearities in the cases that generate more revenue. Lower revenues also lead to smaller changes in the provider's NPV when the percentage value is changed. This is confirmed by examining the slope of the lines.

\section{Servicing Architecture Costs}

Spacecraft costs are very difficult to estimate. Even with the most sophisticated estimation tools, programs often incur cost over-runs. To demonstrate the sensitivity of the servicing architecture costs to the provider's NPV, each of the cases is examined using a $50 \%$ increase and a $25 \%$ decrease in total servicing architecture cost. The results are presented in Figure 5-5. A 50\% decrease in cost was not examined as it seems out of the realm of possibility for these architectures. 


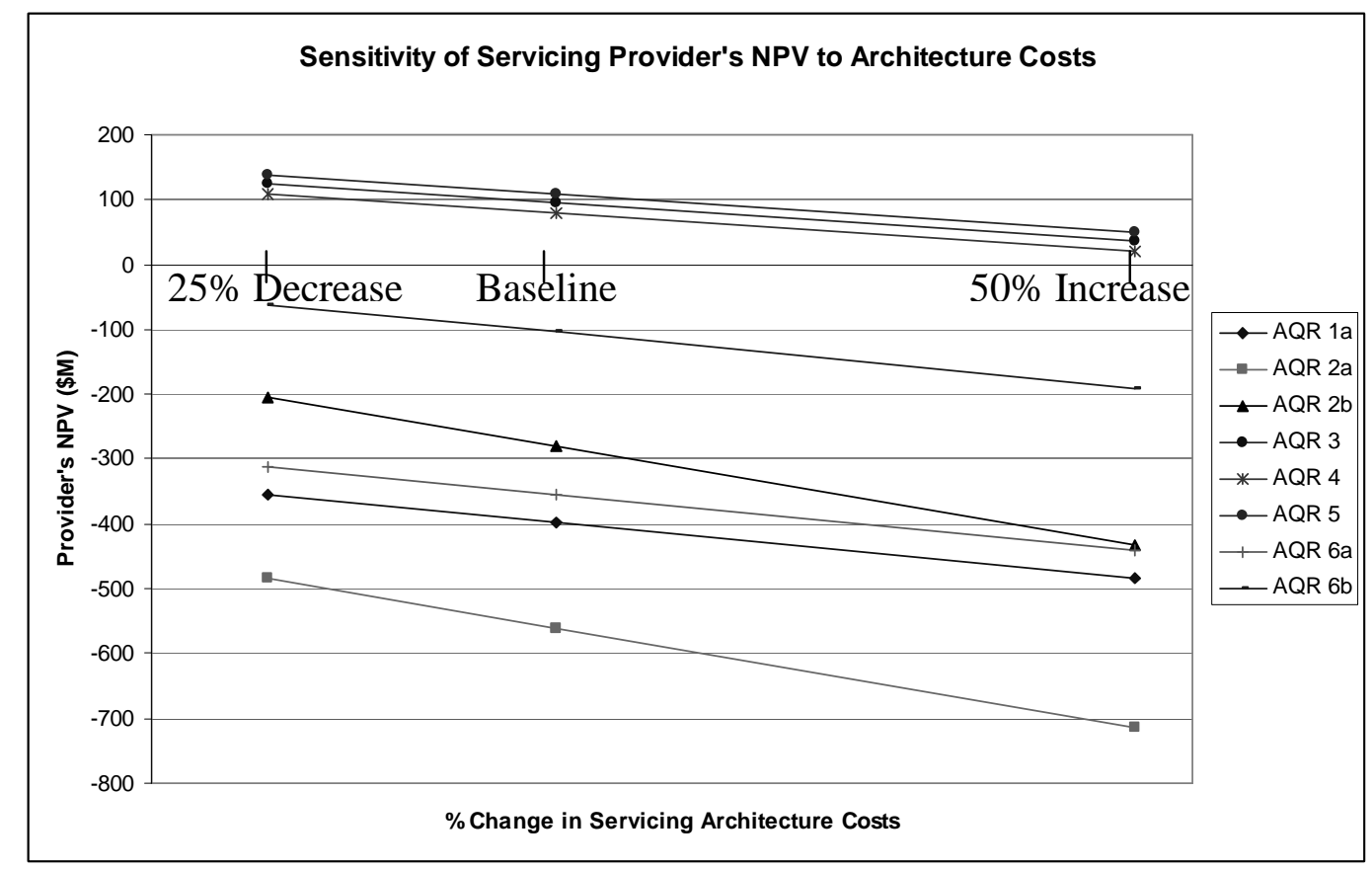

\section{Figure 5-5 Sensitivity of Provider NPV to Servicing Architecture Cost}

As can be seen in Figure 5-5, the servicing provider's NPV has a linear relationship with the servicing architecture cost. The figure also illustrates that the provider's NPV is quite sensitive to the architecture cost. Although this is an expected result, it highlights the importance of having an accurate cost estimate.

\section{Fuel Delivery Costs}

Just as spacecraft costs are difficult to accurately predict, fuel delivery costs are also hard to estimate. In this analysis, they depend on the design, manufacturing, and operating costs of Aquarius, or another low-cost launch vehicle. As these costs are a significant portion of the cost of servicing and have high uncertainty due to the technical risks associated with developing a low-cost launch vehicle, the sensitivity of the provider's NPV to the fuel delivery costs is examined using a 50\% increase and decrease in these costs. 


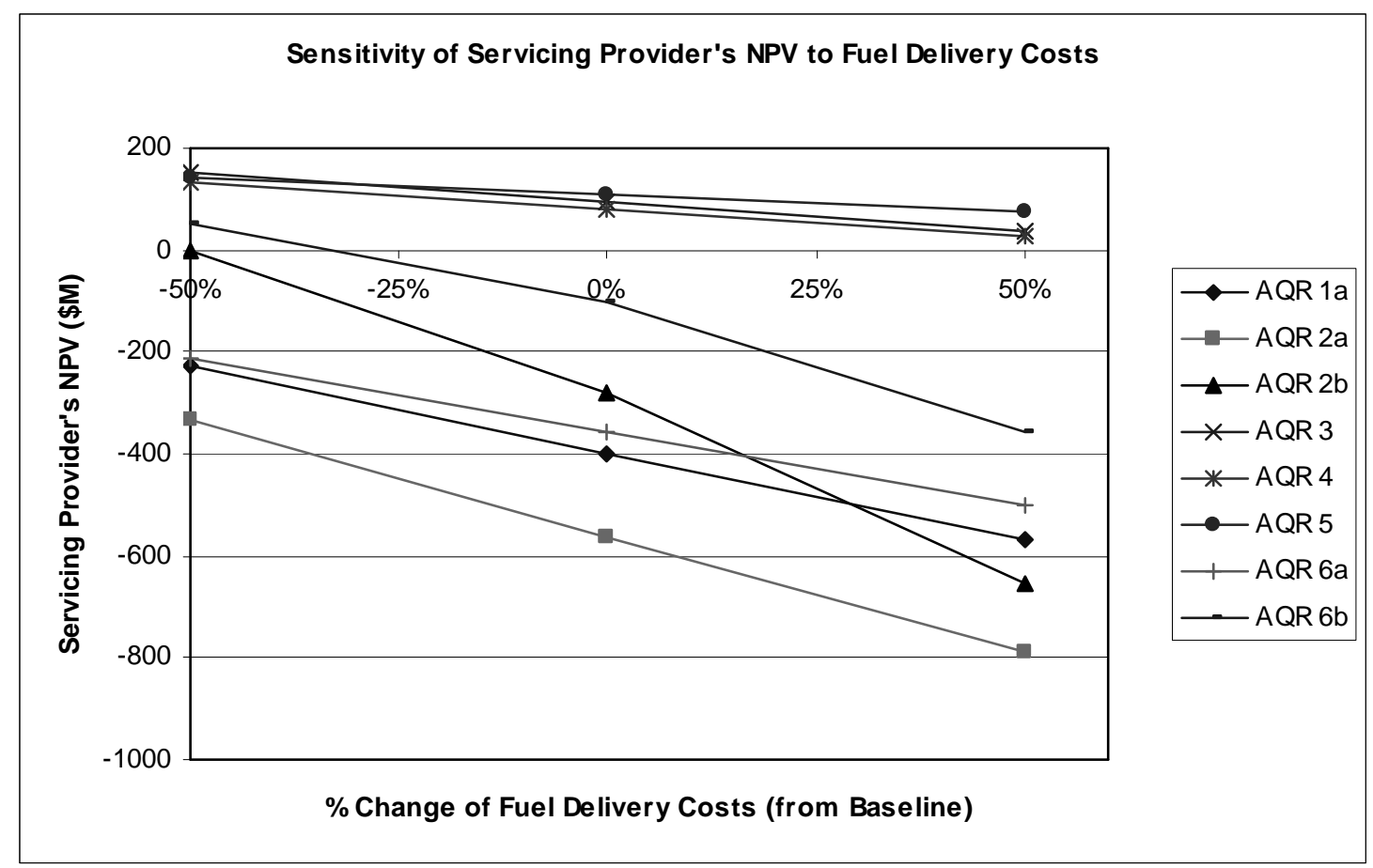

Figure 5-6 Sensitivity of Servicing Provider's NPV to Fuel Delivery Costs

As expected, the servicing provider's NPV decreases as the fuel delivery costs increase. It is interesting to note that the servicing architectures that have positive NPVs with the original fuel delivery costs, continue to have positive NPVs, even with a $50 \%$ increase in fuel delivery costs. The architectures with positive NPVs are the cases that require fuel delivery only in GEO. Thus, these cases are still viable if the fuel delivery launch vehicle used is the Atlas IIAS instead of Aquarius, because the cost difference of fuel delivery is less than the $50 \%$ increase assumed in the sensitivity analysis.

Additionally, AQR $2 \mathrm{~b}$ and $6 \mathrm{~b}$ become economically feasible (i.e. they have positive NPVs) when the fuel delivery costs are decreased by $50 \%$.

\section{Discount Rate}

The discount rate of a project is a very important aspect of the project's valuation. Generally, the determination of the viability of a project, based on the NPV, is significantly affected by the discount rate. As aerospace projects are generally discounted at approximately 10\% ("Cost of Capital by Sector," 2001), only one other 
discount rate, $15 \%$, is used for comparison. This discount rate is carried through the entire analysis, from determining the client's NPV associated with each case, to the servicing provider's NPV calculations. The results are shown in Figure 5-7.

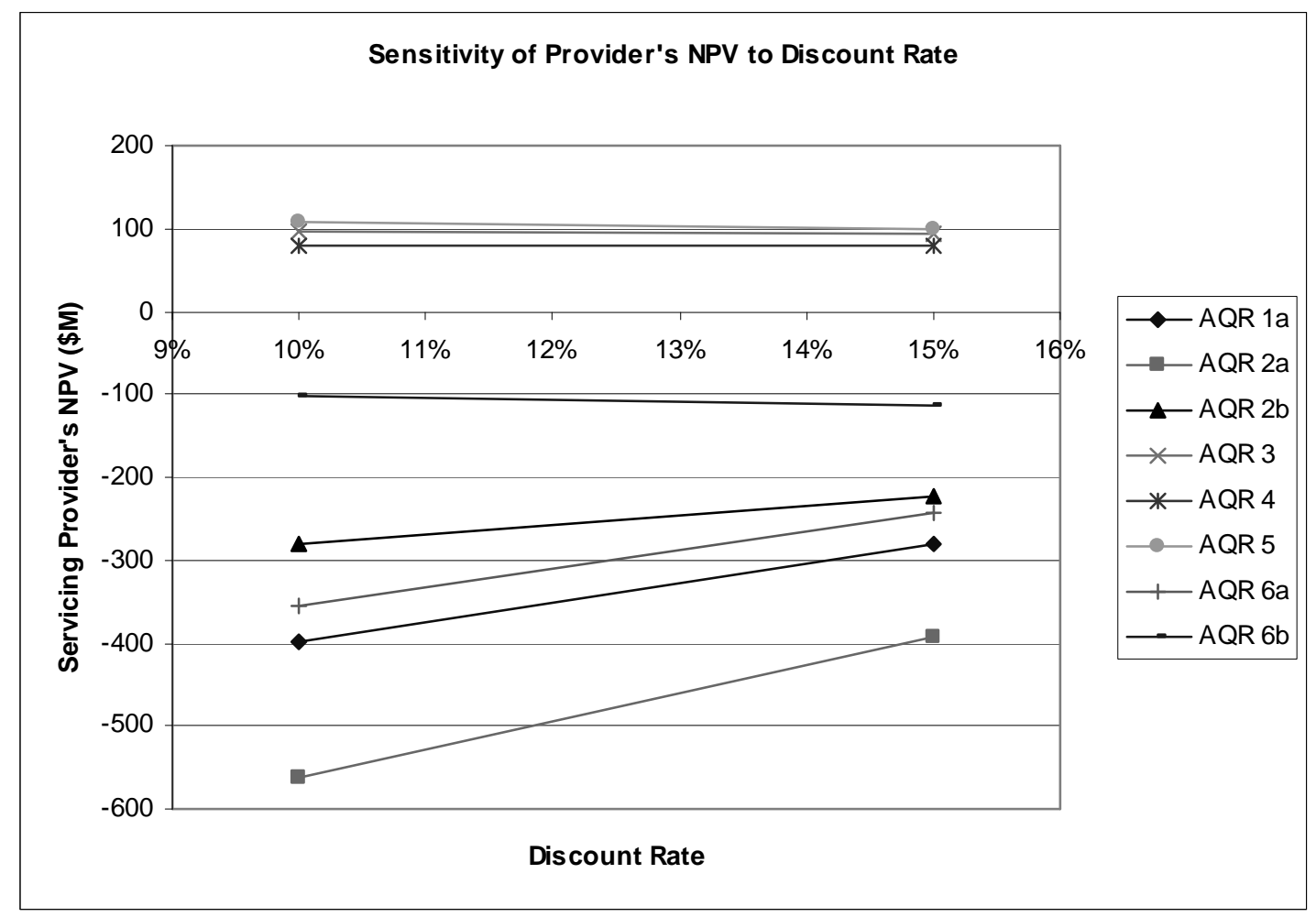

\section{Figure 5-7 Sensitivity of Provider's NPV to Discount Rate}

Increasing the discount rate has, at first glance, unexpected results. In general, when the discount rate is increased, NPV decreases. This is because discounting future cash flows with a higher discount rate decreases their present value. However, in the cases, AQR 1a, $2 \mathrm{a}, 2 \mathrm{~b}$, and $6 \mathrm{a}$, the NPV actually increases with an increased discount rate. This is because these cases have negative NPVs when discounted at $10 \%$. Thus, when the discount rate is increased, the negative cash flows (i.e. costs) are discounted backward and become smaller in magnitude, increasing the overall NPV.

Also unexpected is the lack of sensitivity of $\mathrm{AQR} \mathrm{3,4}$, and 5 to the discount rate. This happens because the potential client's cases are also discounted at 15\%. Although this decreases the client's NPVs, the normalized difference between each case and Comp 1 
(the NPV of the provider's revenues) remains nearly constant. This leads to only a slight decrease in provider NPV for each of these 3 cases.

\subsection{Conclusions}

This chapter completes the economic picture of a potential servicing market by using the information from Chapter 4, the client's perspective on servicing, to determine if there is a potential market for servicing. In addition, if this market exists, it examines what the proper system architecture is and what the provider stands to gain by building this architecture.

The servicing architecture that provides the most economic benefit to the provider is AQR 5, where the serviced satellite is inserted directly into GEO by the launch vehicle and utilizes a refueling satellite to be refueled approximately once per year. The AQR 5 configuration is also the best case from the perspective of the client, after taking into account the technical and economic aspects associated with the case. This indicates there is a market for servicing and that the proper system architecture is Architecture $\mathrm{C}$ (see Table 5-2 and Figure 5-3).

In addition to examining the best servicing architecture, it was found that the cases that have positive NPVs tend to remain positive under the assumptions in the sensitivity analysis. This indicates that these cases, AQR 3, 4, and 5, are the most viable candidates for satellite servicing, as they provide value to both the client and provider even under varying assumptions. The cases that consistently have positive NPVs, indicating their market viability, are all cases that utilize DGI. 



\section{Chapter 6. Conclusion}

This chapter presents a summary of the research presented in this thesis. The primary goal of the research was to develop and apply a new approach to valuation that addresses some of the downfalls of current valuation techniques. This goal is addressed throughout the thesis.

\subsection{Research Conclusions Overview}

\subsubsection{Background}

The thesis began by presenting a background discussion of the Aquarius launch vehicle concept, the topics of real options and flexibility, and previous satellite servicing research. The Aquarius launch vehicle concept was a catalyst for this research, as a low cost launch vehicle is one of the enablers for a satellite servicing market. Including real options in a valuation allows one to properly account for the flexibility inherent in many projects. While previous satellite servicing research has focused primarily on specific architectures for upgrading satellites, this research explored the viability of the commercial GEO satellite servicing market. 


\subsubsection{A New Valuation Technique}

Chapter 2 discussed the downfalls of current valuation techniques. A new valuation technique was presented - one that accounts for the interactions between technology and economics, accounts for the flexibility in a project, and aids in the determination of the "right" product.

\subsubsection{Client Value Analysis for Satellite Servicing}

The valuation technique presented in Chapter 3 was utilized in Chapters 4 and 5 to determine the viability of the GEO satellite servicing market. Chapter 4 presented an analysis of the client's value for various competition and servicing approaches.

The analysis indicates that both servicing and EP provide for increased NPV over the current satellite configuration. Although servicing provides greater NPV than EP, servicing may also increase on-orbit risk. A client's decision between EP and servicing would be based upon both confidence in the reliability of servicing and economic reward. The most economically valuable type of servicing from the perspective of the client is to use a tug spacecraft to perform both OR and NSSK maneuvers for the client spacecraft. However, this case is completely reliant upon the provider, which increases its riskiness. A more viable type of servicing is one that provides value and less risk. The two refueling cases, $\mathrm{AQR} 4$ and 5, accomplish this.

\subsubsection{Provider Value Analysis for Satellite Servicing}

After the client's value for each type of servicing is analyzed, the value to the provider must be considered. Chapter 5 presents this analysis by evaluating the architectures, costs, and revenues associated with each servicing configuration. The value of each case is examined.

The initial and sensitivity analyses indicate that the GEO satellite servicing market is viable. The cases that are most attractive to both the client and the provider are the cases that utilize DGI. Additionally, the assumption of a low-cost launch vehicle was relaxed to determine that the economically viable cases remain viable using the Atlas IIAS for fuel delivery to GEO. After taking into account risk, the recommended method of 
servicing is AQR 5, which utilizes DGI and low frequency (less than one time per year) refueling for stationkeeping. This configuration provides value to both the client and provider and is less reliant upon the provider, reducing the client's risk.

\subsection{Enabling Future Research}

This thesis provides many opportunities for future research in the areas of valuation and satellite servicing. These opportunities include:

- Applying the valuation framework to different systems, including nonaerospace systems

- Adapting the valuation framework to military and civil space systems by using different metrics. (e.g. utility or \% increase in available payload instead of revenues)

- Using the valuation framework to account for other types flexibility inherent in servicing, building upon the satellite servicing analysis presented in this thesis

- Quantitatively accounting for risk in valuations. One approach for this is to estimate the insurance costs associated with different services.

- Improve the cost modeling for servicing

- Investigate the value of other types of satellite servicing, including upgrades and inspection

In addition to those listed above, many promising and uncharted research opportunities remain in the area of on-orbit servicing. 



\section{REFERENCES}

Amram, Martha and Kulatilaka, Nalin. 1999. Real Options: Managing Strategic Investment in an Uncertain World. Boston, MA: Harvard Business School Press.

Bearden, David A. Small Satellite Costs. Crosslink: The Aerospace Corporation magazine of advances in aerospace technology, p. 33-45, Winter 2000/2001.

Boer, Peter F. "Traps, Pitfalls and Snares in the Valuation of Technology." Research Technology Management, no. September-October 1998:54.

Brealey, Richard A. and Myers, Stewart C. 1996. Principles of Corporate Finance, $5^{\text {th }}$ ed. New York: McGraw-Hill.

Christodoulou, Kostis. "Dealing with Satellite Spot Price Volatility." Satellite Finance. Issue 40: p. 42-43, October 2001.

"Commercial Space Transportation Forecasts." Federal Aviation Administration's Associate Administrator for Commercial Space Transportation and the Commercial Space Transportation Advisory Committee, May 2001.

Copeland, Thomas E. and Keenan, Philip T. "How much is flexibility worth?" The McKinsey Quarterly, 1998 Number 2.

"Cost of Capital by Sector."

http://www.stern.nyu.edu/ adamodar/New_Home_Page/datafile/wacc.html. January 18,2001 .

Davinic, N. Chappie, S., Arkus, A., and Greenberg, J. "Spacecraft Modular Architecture Design Study: Cost Benefit Analysis of On-Orbit Satellite Servicing." IAF, $48^{\text {th }}$ International Astronautical Congress (IAA-97-1.4.07), Turin, Italy, October 1997.

"Decisioneering UK: Analyzing Engineering Projects Using Real Options-Intelligent Investments in the Face of Uncertainty." http://www.decisioneering.co.uk/stories/boeing_ro.html. January 23, 2001..

"Delta II Payload Planners Guide." 2000. Huntington Beach, CA: The Boeing Company.

Dixit, Avinash and Pindyck, Robert S. 1994. Investment Under Uncertainty. Princeton, NJ: Princeton University Press.

Draim, Captain J. "The U.S. Navy's HYDRA Project, and Other Floating-Launch Rocket Programs." $48^{\text {th }}$ International Astronautical Congress (IAA-97-IAA.2.2.02), Turin, Italy. October 1997. 
"Federal Reserve Statistical Release", http://www.federalreserve.gov/releases/H15/update. January 23, 2002.

Hall, Eric, and Papadopoulos, Michael. "GPS Structural Modifications for On-Orbit Servicing." Space Technology Conference and Exposition (AIAA-99-4430), Albuquerque, New Mexico, September 1999.

Isakowitz, Steven J., Hopkins, Joseph Jr., Hopkins, Joshua B. International Reference Guide to Space Launch Systems, $3^{\text {rd }}$ ed. 1999. Reston, VA: American Institute of Aeronautics and Astronautics.

Lamassoure, Elizabeth S. "A Framework to Account for Flexibility in Modeling the Value of On-Orbit Servicing for Space Systems." S.M Thesis in Aeronautics and Astronautics at the Massachusetts Institute of Technology. June 2001.

Leisman, Greg and Wallen, Adam. "Design and Analysis of On-Orbit Servicing Architectures for the Global Positioning System Constellation." Master's thesis, Air Force Institute of Technology, March 1999.

Leisman, G., Wallen, A., Kramer, S., and Murdock, W. "Analysis and Preliminary Design of On-Orbit Servicing Architectures for the GOS Constellation." Space Technology Conference and Exposition (AIAA-99-4425), Albuquerque, New Mexico. September 1999.

“The London Satellite Exchange.” http://www.e-sax.com. January 20, 2002.

“Mission Planner's Guide for the Atlas Launch Vehicle Family." 1992. San Diego, CA: General Dynamics Commercial Launch Services.

"Mission Planner's Guide for Atlas Launch Vehicle Family, Rev. 5." 1995. San Diego, CA: Lockheed Martin Commercial Launch Services, Inc.

Morecroft, John D.W. and Sterman, John D (editors). 1994. Modeling for Learning Organizations. Portland, OR: Productivity Press.

Myer, Rudolf X. 1999. Elements of Space Technology. Boston, MA: Academic Press.

Neely III, James E. "Improving the Valuation of Research and Development: A Composite Framework of Real Options, Decision Analysis and Benefit Valuation Frameworks." PhD Thesis in Technology Management and Policy at the Massachusetts Institute of Technology. June 1998.

"Orbital Express Space Operations Architecture/ASTRO."

http://www.darpa.mil/tto/programs/astro.html. February 28, 2002.

“Orbital Express Presentation.” ASTRO ICD Workbook. Space Systems/LORAL. August 3, 2001. 
Pollard, J.E. and Janson, S.W. "Spacecraft Electric Propulsion Applications.” El Segundo, CA: The Aerospace Corporation. February 1, 1996.

Saleh, Joseph H. "Weaving Time Into System Architecture: New Perspective on Flexibility, Spacecraft Design Lifetime, and On-Orbit Servicing." PhD Thesis in Aeronautics and Astronautics at the Massachusetts Institute of Technology. December 2001.

"Space Launch Roundup." Teal Group.

http://www.tealgroup.com/Articles/AeroSpaceAmerica/AeroSpaceAmericaApr01.htm, February 28, 2001.

Trigeorgis, Lenos. 1996. Real Options: Managerial Flexibility and Strategy in Resource Allocation. Cambridge, MA: The MIT Press.

Turner, Andrew E. Ref. 1. "Aquarius Launch Vehicle: Failure is an Option." Space Times: The Magazine of the American Astronautical Society. May/June 2001.

Turner, Andrew E. Ref 2. "Geosynchronous Orbit Direct Injection (GSO) Launch Vehicle Capabilities.” Space Systems/LORAL Internal Memo MU0620-00-124. July $18,2000$.

Turner, Andrew E. Ref 3. "Aquarius: A Low-Cost, Low-Reliability, Existing Technology LV to Orbit Low-Cost Payloads." Space 2000 Conference. (AIAA 20005284). Long Beach, CA. September 2000.

Turner, Andrew E. Ref 4. "Cost-Effective Spacecraft Dependent Upon Frequent NonIntrusive Servicing." Space 2001 Conference. (AIAA 2001-4554). Albuquerque, NM. August 2001.

Waltz, Donald M. 1993. On-Orbit Servicing of Space Systems. Malabar, Florida: Kreiger Publishing Company.

Wertz, James R. and Larson, Wiley J (editors). 1999. Space Mission Analysis and Design $3^{\text {rd }}$ ed. Torrance, CA: Microcosm Press.

Wiesel, William E. 1997. Spaceflight Dynamics $2^{\text {nd }}$ ed. Boston, MA: McGraw-Hill. 



\section{Appendix A}

Satellite Servicing Assumptions and Cases

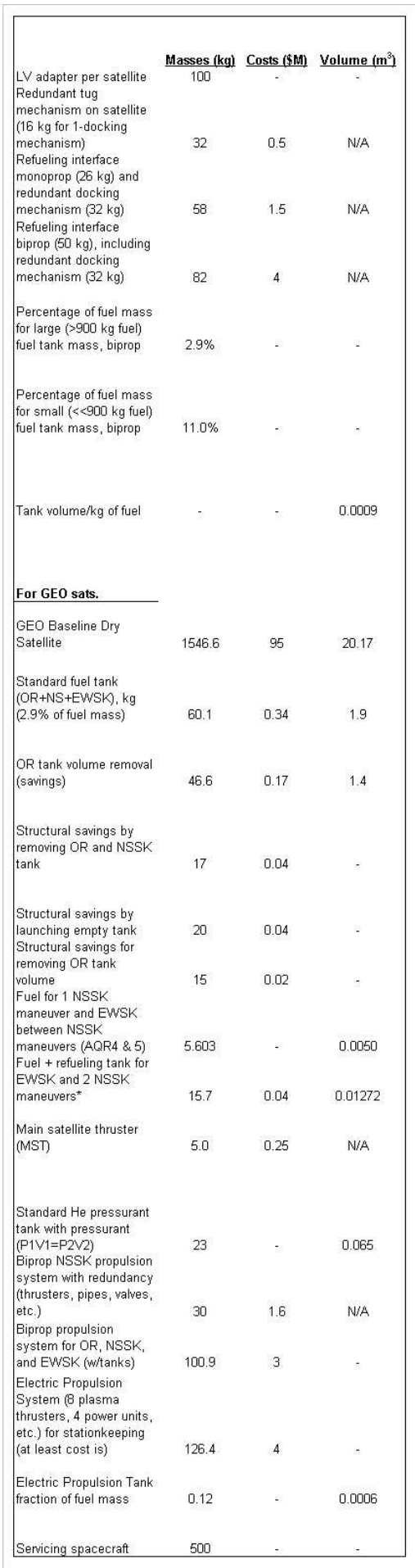
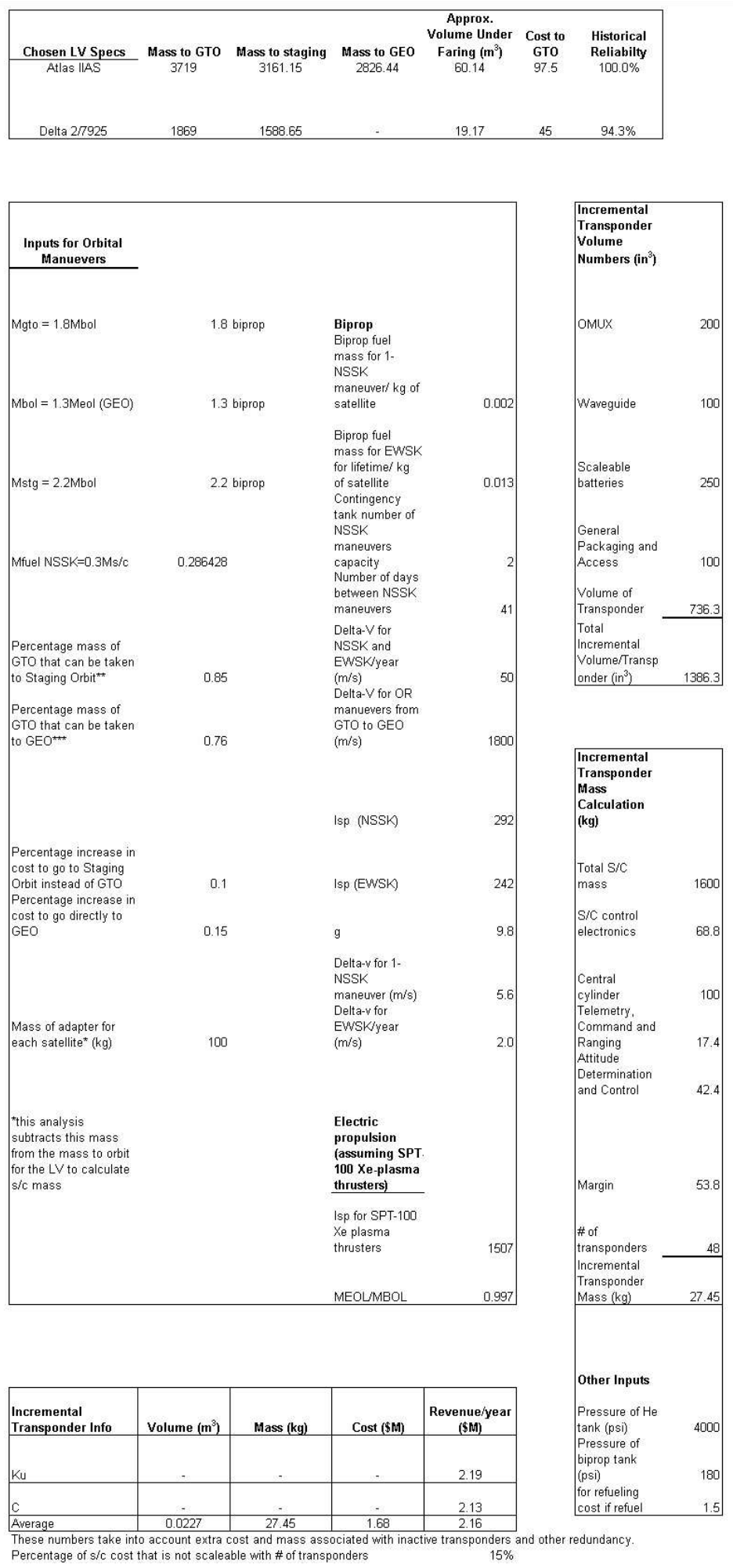
AQR 1a: GEO. Launch dry to staging orbit and fuel 1-time for $O R$ and stationkeeping. 2 spacecraft per launch vehicle

The total volume of the $\mathrm{s} / \mathrm{c}$ has been compared with the total volume of the LV fairing.

AQR $1 \mathrm{a}$ is able to fit 2 redesigned $s / c$ into 1 Atlas IIAS.

Mass Budget

1546.6 Baseline dry mass

Remove standard fuel

-60.1 tanks

79.9 Add new fuel tanks

Remove standard $\mathrm{He}$

-23.0 tank

33.0 Add new He tank

-20.0 Structural mass savings

Refueling and docking

82.0 mechanism

1638.4 Subtotal

Additional available

-157.8 mass

1480.6 Total launched mass

Volume Budget $\left(\mathbf{m}^{3}\right)$

Delete Standard Fuel
-1.9 Tank
Add new fuel tank
2.5 volume
Remove standard He
-0.065 tank
0.094 Add new He tank

0.6 Total change in volume

Cost budget (\$M)

-0.04 Structural savings

4.00 Refueling interface

0.50 Docking mechanism

4.46 Total s/c cost change

Redesign Costs (total

redesign/12

spacecraft)

3.3

Launch Vehicle Costs

97.5 Baseline: ATLAS IIAS

ATLAS IIAS per s/c (2

$53.63 \mathrm{~s} / \mathrm{c})$

-43.9 Change in LC

Total cost change

before additional

transponders

-36.1 (before AQR charges)

Additional Transponder

Limiting factor analysis Incremental Volume/

Transponder $\left(\mathrm{m}^{3}\right)$

Incremental Mass/

Transponder $(\mathrm{kg})$

0.0227

\section{AQR 1a:}

Total available volume

0.0

Total available mass $-157.8$

\# of extra transponders if

volume limited

\# of extra transponders if

mass limited

Total additional

transponders

Cost for additional

transponders

Total additional

revenue/year (\$M)

Total satellite and launch

cost change ( $\$ \mathrm{M})$

Total Revenue/yr (\$M)

Total Cost (\$M)

146.3

Net Present Value (\$M)

169.4 Option to Extend Life

Total Present Value (\$Mi)

229.3

Fuel Budget for delivery (kg)

Fuel to tranfer to 2309.7 GEO

Fuel for NSSK and 444.2 EWSK

Total fuel supplied

2753.9 at staging 


\section{AQR 1b: GEO. Launch dry to staging orbit and fuel 1-time for OR and stationkeeping.}

1 spacecraft in smaller launch vehicle

The total volume of the $s / c$ has been compared with the total volume of the LV fairing.

AQR $1 \mathrm{~b}$ is not able to fit 1 redesigned s/c into 1 Delta 2.

\section{Mass Budget}

1546.6 Baseline dry mass

Remove standard fuel -60.1 tanks

85.7 Add new fuel tanks Remove standard $\mathrm{He}$ -23.0 tank

35.4 Add new He tank

-20.0 Structural mass savings

Refueling and docking

82.0 mechanism

1646.6 Subtotal

Additional available

58.0 mass

1588.7 Total launched mass

Volume Budget $\left(\mathrm{m}^{3}\right)$

Delete Standard Fuel -1.9 Tank

Add new fuel tank

2.7 volume

Remove standard $\mathrm{He}$

-0.065 tank

0.100 Add new He tank

0.8 Total change in volume

Cost budget (\$Mi)

-0.04 Structural savings

4.00 Refueling interface

0.50 Docking mechanism

4.46 Total s/c cost change

Redesign Costs (total redesign/12 spacecraft)

3.33

Launch Vehicle Costs

97.5 Baseline: Atlas IIAS

52.5 Delta $2 / 7925$

-45.0 Change in LC

Total cost change before additional transponders

-37.2 (before AQR charges)

Additional

Transponder Limiting

factor analysis

Incremental Volume/

Transponder $\left(\mathrm{m}^{3}\right)$

Incremental Mass/

Transponder $(\mathrm{kg})$

Case b:

Total available volume $\quad-1.8$

Total available mass

\# of extra transponders if

volume limited

\# of extra transponders if

mass limited

Total additional

transponders

Increased cost for

additional

transponders

Total additional revenue/year (\$Mi)

Total satellite and

launch cost change

(\$M)

\begin{tabular}{|ll|}
\hline $\begin{array}{l}\text { Fuel Budget for } \\
\text { delivery }\end{array}$ & \\
& Fuel to tranfer to \\
2478.3 & GEO \\
& Fuel for NSSK and \\
476.6 & EWSK \\
& Total fuel supplied at \\
& 2954.9 staging \\
\hline
\end{tabular}


AQR 2a: GEO. Launch to staging orbit and tug for OR and NSSK.

tanks removed, $2 \mathrm{~s} / \mathrm{c}$ on one $\mathrm{LV}$

Mass Budget Changes

1546.6 Baseline dry mass Deletion of OR, NSSK,

-60.1 and EWSK fuel tanks Deletion of Main

-5.0 Satellite Thruster Deletion of $\mathrm{He}$

-23.0 pressurant tank Deletion of redundancy -15.0 for NSSK thrusters Contingency and 24.5 EWSK fuel EWSK and contingency 2.7 fuel tank

-17.0 Structural mass savings Addition of tug

32.0 mechanism

1485.7 Subtotal Additional available

5.1 mass

Total allowable

1480.6 launched mass

Volume Budget $\left(\mathrm{m}^{3}\right)$

Deletion of OR, NSSK,

1.87 and EWSK fuel tanks

Deletion of $\mathrm{He}$

0.07 pressurant tank Addition of EWSK and

-0.02 contingency tank

1.91 Total extra volume

Cost budget (\$M)

NSSK \& OR fuel tank

-0.34 savings

Savings from reduction of NSSK redundant

-0.80 thruster system

Deletion of Main

-0.25 Satellite Thruster

Contingency and

0.04 EWSK fuel tank cost

-0.04 Structural savings

0.50 Cost of tug mechanism

Total Change (negative

-0.89 indicates saving \$)

Redesign Costs (total

redesign/12

spacecraft)

3.33

Launch Vehicle Costs

97.5 Baseline: ATLAS IIAS

53.6 ATLAS IIAS to staging (per 1 of $2 \mathrm{~s} / \mathrm{c}$ )

-43.9 Change in LC

Total cost change

before additional

transponders

-41.4 (before AQR charges)
Additional Transponder

Limiting factor analysis

Incremental Volume/

Transponder (m3)

Incremental Mass:

Transponder (kg)

0.0227

Case a:

Total available volume $\quad 0.0$

Total available mass $\quad-5.1$

\# of extra transponders if
volume limited

\# of extra transponders if

mass limited

Total additional

transponders

Cost for additional

transponders

Total additional

revenue/year (\$M)

Total satellite and launch

cost change before

redesign $(\$ M)$

Total Revenue/yr (\$M) $\quad 101.6$

Total Cost (\$M) $\quad 149.4$

Net Present Value $\quad 198.3$

Option to Extend Life $\quad 67.0$

Total Present Value (\$M)

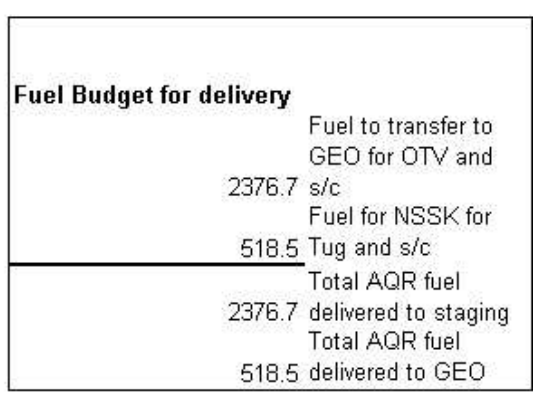


AQR 2b: GEO. Launch to staging orbit and tug for OR and NSSK.

tanks removed, 1 sic on LV, increased payload

Mass Budget Changes

1546.6 Baseline dry mass

Deletion of OR, NSSK,

-60.1 and EWSK fuel tanks

Deletion of Main

-5.0 Satellite Thruster

Deletion of $\mathrm{He}$

-23.0 pressurant tank

Deletion of redundancy

-15.0 for NSSK thrusters

Contingency and EWSK

50.7 fuel

EWSK and contingency

5.6 fuel tank

-17.0 Structural mass savings Addition of tug

32.0 mechanism

$\begin{aligned} 1514.7 & \begin{array}{l}\text { Subtotal } \\ \text { Additional available } \\ \text { mass } \\ \mathbf{1 5 4 6 . 4} \\ \text { Total (LV capacity- } \\ \text { Tot.2 } \\ \text { adapter mass) }\end{array} \\ \text { Volume budget } \mathbf{( m}^{3} \text { ) } & \end{aligned}$

Deletion of OR, NSSK

1.87 and EWSK fuel tanks

Deletion of $\mathrm{He}$

0.07 pressurant tank Addition of EWSK and

-0.05 contingency tank

1.88 Total extra volume

Cost budget

NSSK \& OR fuel tank

-0.34 savings

Savings from reduction

of NSSK redundant

-0.80 thruster system

Deletion of Main

-0.25 Satellite Thruster

Contingency and EWSK

0.04 fuel tank cost

-0.04 Structural savings

0.50 Cost of tug mechanism

-0.89 Total change in $s / c \mathbb{~}$

Redesign Costs (total

redesign $/ 12$

spacecraft)

$$
5.00
$$

Launch Vehicle Costs

97.5 Baseline: ATLAS IIAS

107.3 ATLAS IIAS to STG

9.8 Change in LC

Total cost change before additional

transponders

13.9 (before AQR charges)
Additional Transponder Limiting factor analysis

Incremental Volume/

Transponder (m3)

Incremental Mass/

0.022717844

Transponder ( $\mathrm{kg})$

27.45

Case b:

Total available volume

Total available mass

1546.4

\# of extra transponders if

volume limited

\# of extra transponders if

mass limited

Total additional

transponders

56.3

Increased cost for

additional transponders

Total additional

revenue/year ( $\$ \mathrm{M})$

Total satellite and launch

cost change ( $\$ \mathrm{M})$

108.1

Total Revenue/yr (\$M)

224.8

Total Cost $(\$ \mathrm{M})$

300.6

Net Present Value

459.6

Option to Extend Life

148.7

Total Present Value (\$M)

608.3

Fuel Budget for delivery

Fuel to transfer to GEO for OTV and $4273.4 \mathrm{~s} / \mathrm{c}$

Fuel for NSSK for 932.3 Tug and s/c Total AQR fuel

4273.4 delivered to staging Total AQR fuel 932.3 delivered to GEO 
AQR 3: GEO. DGI wi tug for NSSK.

large tanks removed, $1 \mathrm{~s} / \mathrm{c}$ on $\mathrm{LV}$, increased payload

Mass Budget Changes

1546.6 Baseline dry mass

Deletion of OR, NSSK,

-60.1 and EWSK tank volume

Deletion of Main

-5.0 satellite thrusters

Deletion of pressurant

-23.0 tank

Deletion of redundancy

-15.0 for NSSK thrusters

Contingency and

5.0 EWSK fuel tanks

Contingency and

45.1 EWSK fuel

-17.0 Structural mass savings Addition of tug

32.0 mechanism

1508.6 Subtotal

Additional available

1217.9 mass

Total (LV capacity

2726.4 minus adapter mass)

Volume Budget $\left(\mathrm{m}^{3}\right)$

Deletion of OR, NSSK,

1.87 and EWSK fuel tanks

Deletion of pressurant

0.07 tank

Addition of contingency

-0.01 and EWSK tanks

1.92 Total extra volume

Cost budget (\$Hi)

NSSK \& OR fuel tank

-0.34 savings

0.04 EWSK fuel tank cost

-0.04 Structural savings

0.50 Cost of tug mechanism

Delete Main Satellite

-0.25 Thruster

Deletion of redundancy

-0.80 for NSSK thrusters

Total Change (negative

-0.89 indicates saving $\$$ )

Redesign Costs (total

redesign $/ 12$

spacecraft)

5.00

Launch Vehicle Costs

97.5 Baseline: ATLAS IIAS

112.1 ATLAS IIAS to GEO

14.6 Change in LC

Total cost change

before additional

transponders

18.74 (before AQR charges) 
AQR 4: DGI w/ Just in time refueling. (biprop)

large tanks removed and replaced by refueled tanks, 1 s/c on LV, increased payload

refueled tanks can hold enough for 2 NSSK maneuvers

Number of NSSK maneuvers bettween refuelings

1

Number of times refueled over lifetime of $\mathrm{s} / \mathrm{c}$

Mass Budget Changes

1546.6 Baseline dry mass

Deletion of OR, NSSK,

Additional Transponder

-60.1 and EWSK tank volume Deletion of Main

-5.0 satellite thrusters

Deletion of standard $\mathrm{He}$

-23.0 pressurant tank

Addition of $\mathrm{He}$

10.7 pressurant tank

-17.0 Structural mass savings Mass of refueling and

82.0 docking mechanism

Mass of tanks to be

refueled $w /$ launched

18.7 fuel

1552.8 Subtotal

Additional available

1173.6 mass

Total (LV capacity

2726.4 minus adapter mass)

Volume Budget $\left(\mathrm{m}^{3}\right)$

Deletion of OR, NSSK

1.87 and EWSK fuel tanks Deletion of standard

0.07 pressurant tank Addition of tanks to be

-0.02 refueled

Addition of pressurant

-0.03 tank for refueling

1.88 Total extra volume

Cost budget (\$N)

NSSK \& OR fuel tank

-0.34 savings

Deletion of Main

-0.25 Satellite Thruster

-0.04 Structural savings

0.50 Docking mechanism Cost of refueling

6.00 mechanism

Cost of tanks to be

0.04 refueled

Total Change (negative

5.91 indicates saving $\$$ )

Redesign Costs (total

redesign/12

spacecraft)

5.00

Launch Vehicle Costs

97.5 Baseline: ATLAS IIAS

112.1 ATLAS IIAS to GEO

14.6 Change in LC

Total cost change

before additional

transponders

25.54 (before AQR charges)

0.0227

27.45

\# of extra transponders if

volume limited

\# of extra transponders if

mass limited

Total additional

transponders

42.8

Increased cost additional

transponders

Total additional

revenue/year (\$M)

Total satellite and launch

cost change (\$M)

Total Revenue/yr (\$M)

194.5

Total Cost (\$M)

Net Present Value

380.6

Option to Extend Life

128.7

Total Present Value (\$Mi)

509.3

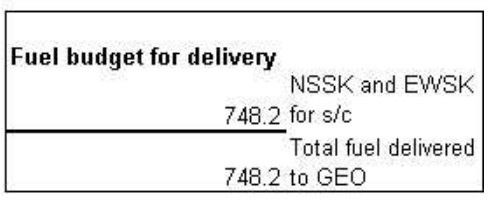


AQR 5: GEO. DGI w/ "Just in time refueling" (varying frequency). (biprop)

large tanks removed and replaced by refueled tanks, $1 \mathrm{~s} / \mathrm{C}$ on LV, increased payload

refueled tanks can hold enough for 2 NSSK maneuvers

Number of NSSK

maneuvers per refueling $\quad 11$

Number of times

refueled over lifetime of

$\mathrm{s} / \mathrm{c}$

Mass Budget Changes

1546.6 Baseline dry mass

Deletion of OR, NSSK

-60.1 and EWSK tank volume

Deletion of Main

-5.0 satellite thrusters

Deletion of standard

-23.0 pressurant tank

Addition of $\mathrm{He}$

10.7 pressurant tank

-17.0 Structural mass savings

Mass of docking and

82.0 refueling mechanisms

Mass of tanks to be

80.9 fuel

1615.0 Subtotal

Additional available

1111.4 mass

Total (LV capacity

2726.4 minus adapter mass)

Volume Budget $\left(\mathrm{m}^{3}\right)$

Deletion of OR, NSSK

1.87 and EWSK fuel tanks

Deletion of standard

0.07 pressurant tank

Addition of tanks to be

-0.07 refueled

Addition of pressurant

-0.03 tank for refueling

1.83 Total extra volume

Cost budget (\$M)

NSSK \& OR fuel tank

-0.34 savings

-0.04 Structural savings

Cost of refueling

6.00 mechanism

Cost of docking

0.50 mechanism

Cost of tanks to be

0.04 refueled

-0.25 Deletion of MST

Total Change (negative

5.91 indicates saving \$)

Redesign Costs (total

redesign/12

spacecraft)

5.00

Launch Vehicle Costs

97.5 Baseline: ATLAS IIAS

112.1 ATLAS IIAS to GEO

14.6 Change in LC

Total cost change before additional transponders

25.54 (before AQR charges)
Additional Transponder

Limiting factor analysis

Incremental Volume/

Transponder $\left(\mathrm{m}^{3}\right)$

Incremental Mass/

Transponder ( $\mathrm{kg}$ )

Total available volume

Total available mass

\# of extra transponders if

volume limited

\# of extra transponders if

mass limited

Total additional

transponders

Increased cost additional

transponders

Total additional

revenue/year ( $\$ \mathrm{M})$

Total satellite and launch

cost change $(\$ \mathrm{M})$

Total Revenue/yr (\$M)

Total Cost (\$M)

Net Present Value

370.3

Option to Extend Life

125.9

Total Present Value (\$M)

Fuel budget for delivery NSSK and EWSK 748.2 for $\mathrm{s} / \mathrm{C}$

Total fuel delivered 748.2 to GEO

assumed to be same regardless of size of tank 
AQR 6a: GEO. Launch to staging, use tug for OR and use EP on orbit for all stationkeeping. 2 spacecraft per launch vehicle

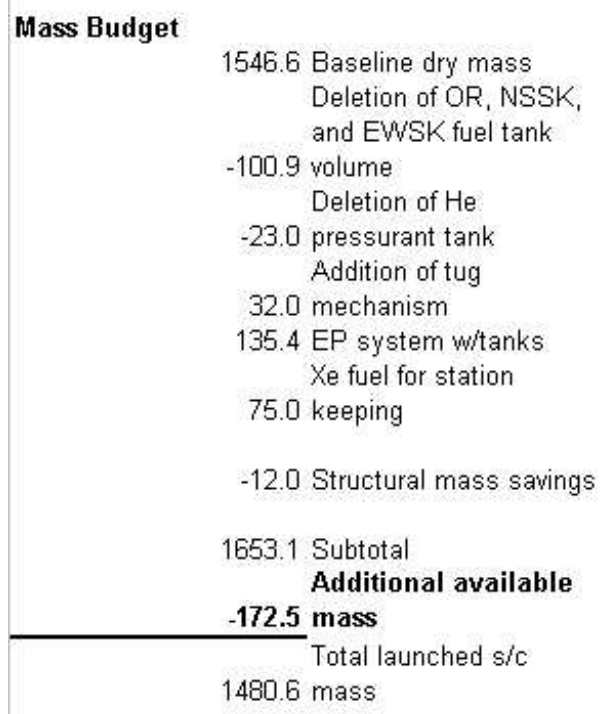

Total cost change before additional transponders

$\mathbf{3 9 . 0 8}$ (before AQR charges)
Additional Transponder Limiting factor analysis

Incremental Volume/

Transponder (m3)

Incremental Mass/

Transponder $(\mathrm{kg})$

Case a:

Total available volume

Total available mass

\# of extra transponders if

volume limited

\# of extra transponders if

mass limited

Total additional

transponders

\section{7}

cost for

additional transponders

Total additional

revenue/year (\$M)

Total satellite and

launch cost change (\$M)

Total Cost (\$M)

Net Present Value

Option to Extend Life

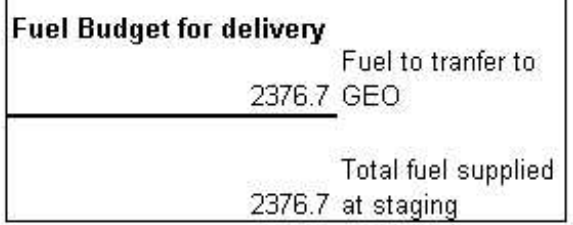


AQR 6b: GEO. Launch to staging, use tug for OR and use EP on orbit for all stationkeepir 1 spacecraft per $L V$, extra payload

Mass Budget

1546.6 Baseline dry mass Deletion of biprop OR and SK propulsion

-100.9 system (w/tanks) Deletion of $\mathrm{He}$

-23.0 pressurant tank Addition of tug

32.0 mechanism

145.0 EP system witanks Xe fuel for station

155.1 keeping

-10.0 Structural mass savings

1744.8 Subtotal

\section{Additional available}

1316.4 mass

Total launched s/c

3061.2 mass

Volume Budget $\left(\mathrm{m}^{3}\right)$

Deletion of OR, NSSK \& EWSK fuel tank

1.865 volume

-0.090 Addition of EP tanks Deletion of pressurant

0.065 tank

1.775 Total extra volume

Cost budget (\$Mi)

Deletion of OR, NSSK and EWSK biprop

-3.00 capabilities Addition of EP for

4.00 stationkeeping Addition of tug

0.50 mechanism

-0.04 Structural savings

1.46 Total $s / c$ cost change

Redesign Costs (total

redesign $/ 12$

spacecraft)

$$
5.00
$$

Launch Vehicle Costs

97.5 Baseline: Atlas IIAS 107.3 Atlas llAS to staging

9.8 Change in LC

Total cost change before additional transponders

16.2 (before AQR charges)
Additional Transponder Limiting factor analysis Incremental Volume/

Transponder $\left(\mathrm{m}^{3}\right)$

Incremental Mass/

Transponder $(\mathrm{kg})$

27.45

Case b:

Total available volume

Total available mass

1316.4

\# of extra transponders if

volume limited

\# of extra transponders if

mass limited

48.0

Total additional

transponders

47

Cost for additional

transponders

Total additional

revenue/year (\$M)

101.6

Total satellite and launch

cost change (\$M)

95.3

Total Revenue/yr (\$M)

205.3

Total Cost ( $\$ \mathrm{M})$

Net Present Value

Option to Extend Life

411.8

135.7

Total Present Value (\$M)

547.5

Fuel Budget for delivery

Fuel to tranfer to 4273.4 GEO

Total fuel supplied 4273.4 at staging 
Comp 1: GEO. DGI w/EP used for stationkeeping. (comparison case, no AQR fuel used) large tanks removed, 1 s/c on LV, increased payload

Mass Budget Changes

1546.6 Baseline dry mass

Deletion of OR, NSSK,

-60.1 and EWSK tank volume

Deletion of biprop

-100.9 propulsion system

Deletion of $\mathrm{He}$

-23.0 pressurant tank

143.0 EP system (tanks, etc.)

Xenon for all

stationkeeping

138.1 maneuvers

-11.0 Structural mass savings

1632.7 Subtotal

Additional available

1093.8 mass

Total ( $\mathrm{V}$ capacity

2726.4 minus adapter mass)

Volume Budget $\left(\mathrm{m}^{3}\right)$

Deletion of standard

OR, NSSK \& EWSK

1.87 fuel tank volume

Deletion of $\mathrm{He}$

-0.07 pressurant tank

0.08 Addition of EP tanks

1.88 Total extra volume

Cost budget (\$Mi)

\begin{tabular}{|c|c|c|}
\hline \multirow[t]{2}{*}{ 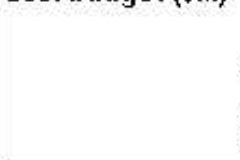 } & -3.00 & $\begin{array}{l}\text { Deletion of biprop } \\
\text { propulsion system }\end{array}$ \\
\hline & $\begin{array}{r}4.00 \\
-0.04\end{array}$ & $\begin{array}{l}\text { EP system (tanks, etc.) } \\
\text { Structural savings }\end{array}$ \\
\hline & 0.96 & $\begin{array}{l}\text { Total Change } \\
\text { (negative indicates } \\
\text { saving \$) }\end{array}$ \\
\hline
\end{tabular}

Redesign Costs (total

redesign/12

spacecraft)

5.00

Launch Vehicle Costs

97.5 Baseline: ATLAS IIAS

112.1 ATLAS $\|$ AS to GEO

14.6 Change in LC
Additional Transponder Limiting factor analysis

Incremental Volume/

Transponder (m3)

Incremental Mass/

0.02

Transponder ( $\mathrm{kg}$ )

27.45

Total available volume

Total available mass

1093.8

\# of extra transponders if

volume limited

82.8

\# of extra transponders if

mass limited

Total additional

transponders

Increased cost additional

transponders

65.6

Total additional

revenue/year ( $\$ \mathrm{M}$ )

Total satellite and launch

cost change (\$M)

86.2

Total Revenue/yr (\$M)

188.1

Total Cost (\$M)

278.7

Net Present Value

368.2

Option for Life Extension

0.0

Total Present Value (\$M)

Total cost change before additional transponders 
Comp 2: SIC launched to GTO. EP used for OR and stationkeeping. (comparison case, no AQR fuel) large tanks removed, $1 \mathrm{~s} / \mathrm{c}$ on LV, increased payload

\section{Mass Budget Changes}

1546.6 Baseline dry mass

Deletion of OR, NSSK,

-46.6 and EWSK tank volume Deletion of biprop

-100.9 propulsion system Deletion of $\mathrm{He}$

-23.0 pressurant tank

195.7 EP system (tanks, etc.) Xenon for all $O R$

415.0 maneuvers

Xenon for all SK

162.3 maneuvers

-11.0 Structural mass savings

2138.0 Subtotal

Additional available 1481.0 mass

Total (LV capacity

3619.0 minus adapter mass)

Volume Budget $\left(\mathrm{m}^{3}\right)$

Deletion of standard

OR, NSSK \& EWSK

1.45 fuel tank volume

Deletion of $\mathrm{He}$

-0.07 pressurant tank

-0.34 Addition of EP tanks

1.05 Total extra volume

Cost budget (\$M)

Deletion of biprop

-3.00 propulsion system

4.00 EP system (tanks, etc.)

-0.04 Structural savings

Total Change

(negative indicates

0.96 saving \$)

Redesign Costs (total

redesign/12

spacecraft)

5.00

Launch Vehicle Costs

97.5 Baseline: ATLAS IIAS

97.5 ATLAS IIAS to GTO

0.0 Change in LC

Total cost change

before additional

transponders
Additional Transponder Limiting factor analysis

Incremental Volume/

Transponder (m3)

0.02

Incremental Mass/

27.45

Total available volume

Total available mass

1481.0

\# of extra transponders if

volume limited

46.1

\# of extra transponders if

mass limited

54.0

Total additional

transponders

Increased cost additional

transponders

Total additional

revenue/year (\$M)

99.4

Total satellite and launch

cost change (\$M)

83.3

Total Revenue/yr (\$M)

203.2

Total Cost ( $\$ M)$

275.8

Time for OR maneuver

(days)

44

If it were biprop instead $\quad 14$

Shift revenues by (days)

408.7

Net Present Value

0.0

Option for Life Extension

408.7

Total Present Value (\$M)
30 